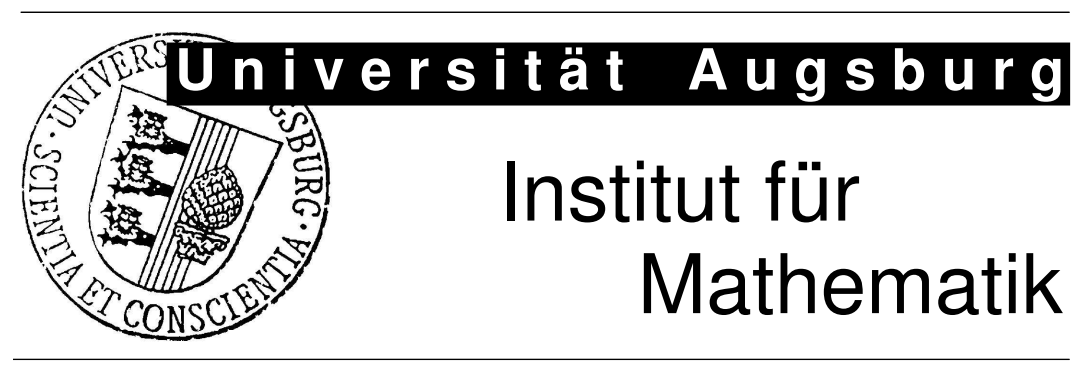

Anda Degeratu, Katrin Wendland

Friendly giant meets pointlike instantons? On a new conjecture by John McKay 


\section{Impressum:}

Herausgeber:

Institut für Mathematik

Universität Augsburg

86135 Augsburg

http://www . math. uni-augsburg.de/forschung/preprint/

ViSdP:

Katrin Wendland

Institut für Mathematik

Universität Augsburg

86135 Augsburg

Preprint: Sämtliche Rechte verbleiben den Autoren (C) 2007 


\title{
Friendly giant meets pointlike instantons? On a new conjecture by John McKay
}

\author{
Anda Degeratu and Katrin Wendland
}

\begin{abstract}
A new conjecture due to John McKay claims that there exists a link between (1) the conjugacy classes of the Monster sporadic group and its offspring, and (2) the Picard groups of bases in certain elliptically fibered Calabi-Yau threefolds. These Calabi-Yau spaces arise as F-theory duals of point-like instantons on ADE type quotient singularities. We believe that this conjecture, may it be true or false, connects the Monster with a fascinating area of mathematical physics which is yet to be fully explored and exploited by mathematicians. This article aims to clarify the statement of McKay's conjecture and to embed it into the mathematical context of heterotic/F-theory string-string dualities.
\end{abstract}

\section{Introduction}

John McKay has observed a remarkable connection between the three sporadic groups: the Monster, the Baby Monster, the Fischer group, and the three affine Dynkin diagrams: $E_{8}, E_{7}$, $E_{6}[\mathrm{McK} 80]$. Let us present this statement in more detail, following Borcherds [Bor02, Bor01] and Glauberman and Norton [GN01].

The Monster group $\mathbb{M}$ has a total number of 194 conjugacy classes, two of which contain elements of order 2 ; we denote them by $2 A$ and $2 B$. The class $2 A$ is the conjugacy class of the Fischer involution in $\mathbb{M}$ so that its centralizer is a double cover of the Baby Monster $\mathbb{B}$. There are 9 conjugacy classes of $\mathbb{M}$ which can be written in the form $\left[t t_{i}\right], i=0, \ldots, 8$, with $t, t_{i}$ of type $2 A$. The orders of $t t_{i} \in \mathbb{M}$ are $1,2,3,4,5,6,2,3,4$, numbers which are familiar as the numbers which label ${ }^{1}$ the affine Dynkin diagram $E_{8}$.

The Baby Monster $\mathbb{B}$ has 5 conjugacy classes of elements which can be written as a product of two elements of type $2 A$. These have orders $2,4,3,2,1$, the numbers labeling the affine Dynkin diagram $F_{4}$. The diagram is related to the affine Dynkin diagram of $E_{8}$ as follows: Omitting the node labeled 2 on the left side of the diagram in the latter gives the $E_{7}$ Dynkin diagram, which we then extend to get its affine version. Folding this by its $\mathbb{Z}_{2}$ automorphism gives the affine Dynkin diagram of $F_{4}$. On the level of the associated sporadic groups the omission of

\footnotetext{
${ }^{1}$ In terms of representation theory, if $\alpha_{1}, \ldots, \alpha_{8}$ denote the fundamental roots in a chosen root system for $E_{8}$, and if $\alpha_{0}$ is the negative of the maximal root of the system, then there exist integers $c_{0}, c_{1}, \ldots, c_{8}$ with $c_{0}=1$, so that $\sum_{i=0}^{8} c_{i} \alpha_{i}=0$. Here $\left(c_{0}, \ldots, c_{8}\right)=(1,2,3,4,5,6,2,3,4)$.
} 
the node labeled 2 corresponds to taking the centralizer of an element of type $2 A$ in $\mathbb{M}$, which gives the Baby Monster $\mathbb{B}$.

The Fischer group $\mathrm{Fi}_{24}$ has 3 classes of elements that are products of two elements of type $2 A$. The orders are $2,3,1$, the numbers labeling the affine Dynkin diagram of $G_{2}$. This diagram is obtained from the affine Dynkin diagram of $E_{6}$ via a folding under a symmetry of order 3 .

John McKay has pointed out another mysterious appearance of the number 194 of conjugacy classes of the Monster, together with various $E_{8}$ 's. 194 occurs as the Picard number of the base in an elliptically fibered Calabi-Yau 3-fold with section, which was studied by Aspinwall, Morrison, and Katz [AM97, AKM00] in the context of the so-called heterotic-F-theory duality. The 3 -fold is the F-theory dual of the $E_{8} \times E_{8}$ heterotic data consisting of 24 pointlike instantons in an $E_{8}$ quotient singularity on a $K 3$ surface. This is the most degenerate situation of a heterotic - F-theory pair: The maximal number of pointlike instantons is moved into the worst possible quotient singularity on $K 3$. Moreover, on the F-theory side, our 3-fold has Euler characteristic 960, the current record among Calabi-Yau 3-folds. McKay's observation adds that on the F-theory side we also find the maximal number of conjugacy classes of a sporadic group... Though the evidence may be scarce, if McKay's numerology is true, then this points to a very interesting connection: surface orbifold singularities for the exceptional simply-laced Dynkin diagrams should correspond to elliptically fibered Calabi-Yau 3-folds over rational surfaces with Picard number equal to the number of conjugacy classes in the three sporadic simple groups.

Motivated by these observations, we originally set out to prove or disprove McKay's conjecture. Using the results of [AM97] it is not too hard to see that the conjecture at least needs refinement, since 24 pointlike instantons on other ADE type singularities under the heterotic - F-theory duality do not produce any convincing numerology. In fact, because $F_{4}$ and $G_{2}$ are non-simply laced but give the Dynkin data corresponding to the Baby Monster and the Fischer group, any naive attempt to collect further evidence for McKay's conjecture by placing pointlike instantons on other rational double points was bound to fail. We will briefly comment on possible remedies in the Conclusions. However, instead of ending this work here by announcing that McKay's conjecture as yet awaits confirmation by further data points, we prefer to report on the fascinating areas of mathematical physics which this very conjecture relates to: On our journey we quickly got entangled in the amazing features of string-string dualities. Though friendly giants have not yet been sighted, pointlike instantons will certainly make their appearance in this work. In summary, the reader should be warned that the title of this work can be misleading: It honestly states the outset of this project but does not reflect the fact that the pointlike instantons or rather the heterotic - F-theory duality which supposedly confronts them with the friendly giant is playing the main part in our study. We aim to give a mathematical account of some foundations of string-string duality and include some of the open questions which we plan to address in future work. Much of our discussion is collected from the vast literature on this topic. However, we attempt to carefully separate physics lore from mathematical derivations, to pinpoint the open questions, to explain computations in algorithmic form, and to illustrate them with examples where appropriate, in a form which is not yet available in the literature. A number of original observations are found in this work, but on the large this is a review article. We hope to convince the reader that this field of mathematical physics deserves more attention than it has so far received from mathematicians. 
The paper is organized as follows:

In Section 2 we present aspects of heterotic $E_{8} \times E_{8}$ and type IIA string theory which are relevant for our later discussion. The main focus is on the form of the massless spectrum; we describe first the massless spectrum of the $D=10$ theories, and then its modification as we compactify to a lower dimensional theory. We treat the cases when the compactifying manifolds are $K 3$ surfaces, $T^{4}, K 3 \times T^{2}$, and smooth Calabi-Yau 3-folds, and in each of these cases we determine the numbers $n_{V}, n_{T}, n_{H}$ of linearly independent vector-, tensor-, and hypermultiplets in the massless spectrum. More precisely, we study carefully the behavior of the Yang-Mills multiplet in 10-dimensional heterotic theories under compactification. We modify an Atiyah-HitchinSinger index theorem [AHS78, Thm. 6.1] to relate the multiplicities arising in this context to the dimensions of the moduli spaces of anti-selfdual connections on $E_{8}$-bundles over $K 3$. These moduli spaces must have non-negative dimensions to yield consistent theories, imposing bounds on the instanton numbers of the bundles in play. Although these observations must be known to physicists, we have not been able to find explanations, along the lines we are giving, in the literature. We also discover a correction to the count of neutral versus charged massless hypermultiplets which seems to have escaped mention so far.

In Section 3 we discuss the anomaly cancellation condition, which in physics arises as a consistency condition for string theory. Our focus is the heterotic $E_{8} \times E_{8}$ theory compactified on a $K 3$ surface, where this condition takes the form

$$
n_{H}-n_{V}+29 n_{T}=273 .
$$

We give two ways to derive it: (1) directly on the space-time, and (2) on the $K 3$ surface by employing a purely index theoretical argument. It is not clear from the literature whether the physics community is aware of the equivalence of (1) and (2). We end the section by discussing a number of examples. Similar (and more extensive) collections of examples have appeared previously, see particularly $\left[\mathrm{BIK}^{+} 96\right]$; our list is representative in view of the later comparison to purely geometrical techniques which are shown to reproduce the numbers $n_{H}, n_{V}, n_{T}$ in Section 5.3.

Section 4 presents first the conjectured duality between heterotic and type IIA theories. In the so-called F-theory limit a duality is induced between heterotic $E_{8} \times E_{8}$ string theory compactified on a $K 3$ surface and F-theory compactified on a Calabi-Yau 3-fold which is $K 3$-fibered. The existence of such a limit with the desired properties requires the Calabi-Yau 3-fold to be elliptically fibered, with a section, over a rational surface. The duality predicts that the numbers of linearly independent vector-, tensor-, and hypermultiplets of two dual theories agree.

In Section 5 we analyze the implications of this conjecture from the perspective of the geometry of the elliptically fibered Calabi-Yau 3-fold, and we explain how the above mentioned anomaly cancellation condition (on the heterotic side) induces a classically unknown relation among the geometric invariants of the Calabi-Yau 3-fold (on the F-theory side). The significance of this interpretation of the duality was first observed in [GM03]. We give a detailed summary of this latter work. Particularly, incorporating the more general results of [Mir83], we describe an algorithm to calculate the Euler numbers of these Calabi-Yau 3-folds. This includes a brief discussion of "charged hypermultiplets", together with some new evidence for the geometric realization of these mysterious structures. We plan to give a more complete construction in 
the near future. We end this section by presenting examples where we check that in each case the results match the data on the heterotic side. We also include examples where the heterotic analysis is impossible, because the bundles degenerate in a way which still needs to be understood: the case of pointlike instantons on ADE type quotient singularities on a $K 3$ surface which was pioneered in [AM97]. We give details of the geometric analysis on the F-theory side, most of which must have been known to the authors of [AM97], but which have not appeared elsewhere.

We conclude with a discussion in Section 6 where we take stock of our results, relate them back to McKay's conjecture, and suggest some further steps to take.

Two appendices summarize background material on Rarita-Schwinger fields and on characteristic classes, particularly for $K 3$ surfaces.

\section{Acknowledgements}

John McKay deserves our gratitude for stating a conjecture which has led us into a fascinating area of mathematical physics, and for his steady support and interest. We are grateful to Miles Reid, who was the first to inform us of this conjecture. We thank Gavin Brown, Terry Gannon, Yang-Hui He, Vishnu Jejjala, and Ilarion Melnikov for discussions and encouragement, and particularly Paul Aspinwall, Antonella Grassi, Mark Gross, David Morrison, and Mark Stern for their comments and explanations.

For their hospitality we wish to thank the following institutes, where part of this work was performed: The Isaac Newton Institute in Cambridge, UK, during the programme on "Higher Dimensional Complex Geometry", the "Mathem. Forschungsinstitut Oberwolfach" during the mini workshop "Complex Geometry: Mirror Symmetry and Related Topics", and the Max Planck Institute for Mathematics in Sciences in Leipzig, Germany (AD). We gratefully acknowledge support from the AMS under an AWM travel grant for a visit of AD to the Mathematics Institute of the University of Warwick, UK, whose hospitality also deserves our thanks.

AD was partly supported by NSF grant number DMS-0505767. KW was partly supported by Nuffield Foundation grant number NAL/00755/G.

\section{Vocabulary from heterotic and type IIA theories}

Let us present some of the standard lore of superstring theory. Consistency conditions require one to work on a ten-dimensional real space-time with Minkowski signature, where there are five basic theories: two $\mathcal{N}=2$ (type IIA and type IIB), and three $\mathcal{N}=1$ theories (heterotic $E_{8} \times E_{8}$, heterotic $S O(32)$, and type I). At first level the difference between these theories is given by the number $\mathcal{N}$ of supersymmetries, and by their "massless field content". In fact, when space-time is the ten dimensional Minkowski space, then this information is sufficient to tell the five theories apart, and since we are not capable to address string theory in general, we will focus on issues related to these massless particles.

Interesting structure arises when the space-time is of the form $M^{1, D-1} \times X^{d}$, where $d+D=10$, $M^{1, D-1}$ is flat $D$-dimensional Minkowski space, and the "internal" $X^{d}$ is a real $d$-dimensional 
manifold which admits Ricci-flat Kähler-Einstein metrics. On $M^{1, D-1}$ it is convenient to work with light-cone coordinates, i.e. with $x^{ \pm}=\left(x^{0} \pm x^{D-1}\right) / \sqrt{2}$ and the remaining $(D-2)$ transverse directions, which are space-like. The massless particles are labeled by irreducible representations of $\operatorname{Spin}(D-2)$, the double cover of the little group $\operatorname{SO}(D-2)$, which acts on the transverse directions. Both have the same Lie algebra $\mathfrak{s o}(D-2)$ which we shall use for convenience from now on.

In this section we describe the massless field content of our main protagonists, namely the type IIA and heterotic $E_{8} \times E_{8}$ string theories. We do this first on $M^{1,9}$ and then compactify to $M^{1, D-1} \times X^{d}$ with $D=6$ and $D=4$, respectively.

Much of this section consists of standard material [GSW87, Pol98]. Our exposition aims to present the mathematical details, some of which we have not been able to find explicitly in the literature.

\subsection{Massless spectra in $D=10$}

As stated above, massless particles in 10 dimensions are given by irreducible representations of $\mathfrak{s o}(8)$. Let $\mathbf{8}_{v}, \mathbf{8}_{+}, \mathbf{8}_{-}$denote the real vector, the positive, and the negative real spinor representation of $\mathfrak{s o}(8)$. Spinors transforming in $\mathbf{8}_{ \pm}$are called Majorana-Weyl spinors (see e.g. [Pol98, II, Appendix B] for a useful account on spinors and supersymmetry in various dimensions). Recall that $\mathbf{8}_{v}, \mathbf{8}_{+}, \mathbf{8}_{-}$are related to one another by the triality automorphism of $\mathfrak{s o}(8)$ [Stu03]. For each of the superstring theories, the massless particles arise as tensor products of "left" and "right moving" representations, where the left hand side is always $\mathbf{8}_{v} \oplus \mathbf{8}_{+}$.

\section{Type IIA for $D=10$}

In type IIA theories the massless spectrum is $\left(\mathbf{8}_{v} \oplus \mathbf{8}_{+}\right) \otimes\left(\mathbf{8}_{v} \oplus \mathbf{8}_{-}\right)$. Expanding into irreducible representations of $\mathfrak{s o}(8)$, one has the explicit field content, organized into four sectors with the following standard notations:

\begin{tabular}{|c|c|c|c|c|}
\hline or: & $\mathbf{8}_{v} \otimes \boldsymbol{8}_{v}$ & $=\mathbf{3 5} \oplus \mathbf{2 8} \oplus \mathbf{1}$ & $\rightarrow$ & $g_{M N} \oplus B_{M N} \oplus \phi$ \\
\hline -NS & $\mathbf{8}_{+} \otimes \mathbf{8}_{v}$ & $=5 \mathbf{6}_{+} \oplus \mathbf{8}_{-}$ & $\rightarrow$ & $\Psi_{M}^{+} \oplus \Psi^{-}$ \\
\hline-1 & $\mathbf{8}_{v} \otimes \mathbf{8}_{-}$ & $=56_{-} \oplus 8_{+}$ & $\rightarrow$ & $\Psi_{M}^{-} \oplus \Psi^{+}$ \\
\hline$-\mathrm{B}$ sec & $8_{+} \otimes 8_{-}$ & $=\mathbf{5 6}_{v} \oplus \boldsymbol{8}_{v}$ & $\rightarrow$ & $C_{M N P} \oplus C_{M}$ \\
\hline
\end{tabular}

Here, $g_{M N}$ gives the metric on $M^{1,9}$ up to scaling and is called the graviton; the 2-form $B_{M N}$ on $M^{1,9}$ is the B-field ${ }^{2}$; the scalar $\phi$ is the dilaton; the two fields $\Psi_{M}^{ \pm}$in the $\mathbf{5 6}_{ \pm}$are the MajoranaWeyl gravitinos of opposite chiralities; the $\Psi^{\mp}$ are Majorana-Weyl dilatinos; $C_{M N P}$ is called RR 3-form, $C_{M}$ is an RR 1-form. The graviton has spin 2, all $p$-forms have spin 1, while the gravitinos have spin $\frac{3}{2}$ and pure spinors have spin $\frac{1}{2}$. The fields $\Psi_{M}^{ \pm}$with spin $\frac{3}{2}$ carry both vector and spinor indices; in the physics literature such fields are called Rarita-Schwinger fields, see Appendix A for more details.

\footnotetext{
${ }^{2}$ The B-field is only locally given by a 2-form, as shall be of importance in Section 3.2.1. For the time being, however, we can safely view $B_{M N}$ as a 2 -form on $M^{1,9}$.
} 
Remark. Consider the group $\operatorname{Spin}(n)($ or $\operatorname{Spin}(1, n+1))$. If $n \equiv 0(\bmod 4)$ then the smallest real irreducible representation of the real Clifford algebra $\mathrm{Cl}(n)$ decomposes into two inequivalent representations. On the other hand if $n \equiv 0(\bmod 2)$ then the smallest irreducible complex representation of the complex Clifford algebra $\mathbb{C l}(n)$ decomposes into two inequivalent complex irreducible representations. If these last two representations are self-dual, and if they allow a real structure such that their real part is a real spin representation, then spinors in those real representations are called Majorana-Weyl spinors (for $\operatorname{Spin}(n)$, these are normally called pseudoreal, but we will not make this distinction). From Bott periodicity and the classification of these representations for small $n$, it can be seen that the condition for the existence of Majorana-Weyl spinors is a dimensional one: $n \equiv 0(\bmod 8)$. For example, for $\operatorname{Spin}(8)$ let $\mathbf{8}_{ \pm}$ denote the real spin irreducible representations of real dimension 8 , and let $\mathbf{8}_{ \pm}^{\mathbb{C}}$ denote the complex spin irreducible representations. Then the Majorana-Weyl condition gives that

$$
\mathbf{8}_{ \pm}^{\mathbb{C}}=\mathbf{8}_{ \pm} \otimes_{\mathbb{R}} \mathbb{C}, \quad \mathbf{8}_{ \pm}=\operatorname{Re}\left(\mathbf{8}_{ \pm}^{\mathbb{C}}\right) .
$$

\section{Heterotic $E_{8} \times E_{8}$ for $D=10$}

The massless spectrum of heterotic $G_{10}=E_{8} \times E_{8}$ strings is $\left(\mathbf{8}_{v} \oplus \mathbf{8}_{+}\right) \otimes\left(\mathbf{8}_{v} \oplus \operatorname{Ad}\left(G_{10}\right)\right)$, such that there is supersymmetry only on the left hand side, with the following two irreducible representations of the $\mathcal{N}=1$ super Poincaré algebra:

$$
\begin{array}{ll}
R(10): \quad\left(\mathbf{8}_{v} \oplus \mathbf{8}_{+}\right) \otimes \mathbf{8}_{v} & =\mathbf{3 5} \oplus \mathbf{2 8} \oplus \mathbf{1} \oplus \mathbf{5} \mathbf{6}_{+} \oplus \mathbf{8}_{-} \\
Y(10): & \left(\mathbf{8}_{v} \oplus \mathbf{8}_{+}\right) \otimes \operatorname{Ad}\left(G_{10}\right)=\left(\mathbf{8}_{v} \otimes \operatorname{Ad}\left(G_{10}\right)\right) \oplus\left(\mathbf{8}_{+} \otimes \operatorname{Ad}\left(G_{10}\right)\right) \rightarrow A_{M} \oplus \Lambda^{+}
\end{array}
$$

The irreducible representations of the super Poincaré algebra along with their decomposition under $\mathfrak{s o}(D-2)$ are commonly called supermultiplets. Above, $R(10)$ is the supergravity multiplet, which agrees with the NS-NS plus the R-NS sector of type IIA, containing the graviton $g_{M N}$, the B-field $B_{M N}$, the dilaton $\phi$, the gravitino $\Psi_{M}^{+}$, and the dilatino $\Psi^{-}$. Second, $Y(10)$ is the super-Yang-Mills multiplet, where $A_{M}$ is a gauge field, i.e. a connection 1-form on a principal $G_{10}$-bundle on $M^{1,9}$. For definiteness we always identify the spin connection with the gauge connection. The superpartner $\Lambda^{+}$of $A_{M}$ is called gaugino and is a positive spinor on $M^{1,9}$ with values in the adjoint representation of $G_{10}$. We will see later that under compactification to $K 3$ anomaly cancellation forces the instanton number of the connection $A_{M}$ to be 24 , see $(2.2 .22)$ and Section 3.

\subsection{Compactification}

Let us now "compactify" $d$ dimensions, that is consider the above-mentioned $M^{1, D-1} \times X^{d}$ as background for our strings. We will refer to $M^{1, D-1}$ as space-time, and here assume that the compact space $X^{d}$ is smooth. Space-time indices are denoted by $\mu, \nu$, while coordinates on $X^{d}$ are indexed by $i, j$. We summarize the discussion in [GSW87, II, p. $366 \mathrm{ff}]$.

The massless fields of the ten-dimensional theory decompose under the action of $\mathfrak{s o}(D-2) \oplus$ $\mathfrak{s o}(d)$, and we view the components as fields on the Minkowski space $M^{1, D-1}$. At the same time they give sections in certain fiber bundles on $X^{d}$, depending on the type of field under consideration, together with associated geometrical differential operators, which can be of Dirac-type, 
Laplacians, or Yang-Mills or Einstein linearizations. These operators are "mass operators", and massless fields are characterized as sections in their kernels.

As a representative case consider spinor fields and the corresponding Dirac operator. The above tells us that we need to calculate the kernel of a Dirac operator $\not D$ on $X$. In fact, pairs of elements of opposite helicity in this kernel can conspire and acquire mass, and it is believed that they tend to do so in nature unless a large gauge group remains unbroken, see [GSW87, II, p. 368]. Hence generically to calculate the dimension of the space of massless spinors we determine the index of the appropriate Dirac operator on the compactifying manifold $X$ : This index gives the number of independent solutions to the massless equations of motion which cannot become massive. In other words, a positive index of $\not D$ implies that, when viewed as fields on $X$, we have ind $\not D$ massless fields having positive chirality, while a negative index implies that we have ( - ind $\not D)$ massless fields of negative chirality.

Similar comments hold for the other fields: Rarita-Schwinger fields, $p$-forms, gauge fields, or gravitational fields, where $p$-forms give massless fields iff they are harmonic. Some massless fields satisfy linear equations and these are easy to figure out using the appropriate index theorem on the compactifying manifold $X$. On the other hand, there are fields like the gauge fields and the gravitational fields which satisfy non-linear equations [GSW87, II, p. 398]: the Ricci-flat Einstein equation for the metric, and the Yang-Mills equation for the gauge fields. To count the number of independent massless solutions, one considers the kernel of the linearizations of these operators around a solution.

\subsubsection{Compactification to $D=6$}

Consider superstring theory on $M^{1,5} \times X^{4}$. We need to decompose $\boldsymbol{8}_{v}, \boldsymbol{8}_{+}$, and $\boldsymbol{8}_{-}$under the action of $\mathfrak{s o}(4) \oplus \mathfrak{s o}(4) \subset \mathfrak{s o}(8)$. The vector representation decomposes as

$$
\mathbf{8}_{v}=(\mathbf{4}, \mathbf{1}) \oplus(\mathbf{1}, \mathbf{4})
$$

where 4 is the natural 4 -dimensional vector representation of $\mathfrak{s o}(4)$. To figure out how the spinor representations decompose we need to look first at the decomposition of the complexifications. Under $\mathfrak{s o}(4) \oplus \mathfrak{s o}(4)$ we have

$$
\mathbf{8}_{+}^{\mathbb{C}}=\left(\mathbf{2}_{+}^{\mathbb{C}}, \mathbf{2}_{+}^{\mathbb{C}}\right) \oplus\left(\mathbf{2}_{-}^{\mathbb{C}}, \mathbf{2}_{-}^{\mathbb{C}}\right), \quad \mathbf{8}_{-}^{\mathbb{C}}=\left(\mathbf{2}_{+}^{\mathbb{C}}, \mathbf{2}_{-}^{\mathbb{C}}\right) \oplus\left(\mathbf{2}_{-}^{\mathbb{C}}, \mathbf{2}_{+}^{\mathbb{C}}\right),
$$

where $\mathbf{2}_{ \pm}^{\mathbb{C}}$ are the complex irreducible spin representations of $\mathfrak{s o}(4)$, which have complex dimension 2 each. There are also two real irreducible spin representations $\boldsymbol{4}_{ \pm}$of $\mathfrak{s o}(4)$ of dimension 4 each, which are related to the respective complex spin representations by

$$
\mathbf{4}_{ \pm}=\operatorname{Re}\left(\mathbf{2}_{ \pm}^{\mathbb{C}} \otimes \mathbb{C}^{2}\right)
$$

The above and formula (2.1.2) give

$$
\mathbf{8}_{+}=\mathbf{4}_{++} \oplus \mathbf{4}_{--}, \quad \mathbf{8}_{-}=\mathbf{4}_{+-} \oplus \mathbf{4}_{-+},
$$

where the double indices refer to the behavior of the real four dimensional representations under the respective $\mathfrak{s o}(4)$ actions. 
To summarize, under $\mathfrak{s o}(4) \oplus \mathfrak{s o}(4)$ we have

$$
\mathbf{8}_{v}=(\mathbf{4}, \mathbf{1}) \oplus(\mathbf{1}, \mathbf{4}), \quad \mathbf{8}_{+}=\left(\mathbf{4}_{++}\right) \oplus\left(\mathbf{4}_{--}\right), \quad \mathbf{8}_{-}=\left(\mathbf{4}_{+-}\right) \oplus\left(\mathbf{4}_{-+}\right) .
$$

Below, we determine the massless field content arising from compactification of ten-dimensional string theories to six dimensions. These fields are conveniently grouped into supermultiplets of the respective superalgebras. The following multiplets can arise in our setting [Sei88], where we give the contribution from each irreducible representation of the space-time-so(4):

- in six-dimensional theories with $\mathcal{N}=(1,1)$ supersymmetry,

$$
\begin{array}{lll}
\text { supergravity multiplet: } & \text { bosonic: } & (\mathbf{9}) \oplus 4(\mathbf{4}) \oplus\left(\mathbf{3}_{+}\right) \oplus\left(\mathbf{3}_{-}\right) \oplus(\mathbf{1}), \\
& \text { fermionic: } & \left(\mathbf{1 2}_{+}\right) \oplus\left(\mathbf{1 2}_{-}\right) \oplus\left(\mathbf{4}_{+}\right) \oplus\left(\mathbf{4}_{-}\right), \\
\text {matter multiplet: } & \text { bosonic: } & (\mathbf{4}) \oplus 4(\mathbf{1}), \\
& \text { fermionic: } & \left(\mathbf{4}_{+}\right) \oplus\left(\mathbf{4}_{-}\right) ;
\end{array}
$$

- in six-dimensional theories with $\mathcal{N}=1$ supersymmetry (notations as in [Wal88, Sch96])

$$
\begin{array}{llcl}
R(6) & \text { supergravity multiplet: } & (\mathbf{9}) \oplus\left(\mathbf{1 2}_{+}\right) \oplus\left(\mathbf{3}_{+}\right) & \rightarrow g_{\mu \nu} \oplus \psi_{\mu}^{+} \oplus B_{\mu \nu}^{+}, \\
T(6) & \text { tensormultiplet: } & \left(\mathbf{3}_{-}\right) \oplus\left(\mathbf{4}_{-}\right) \oplus(\mathbf{1}) & \rightarrow B_{\mu \nu}^{-} \oplus \psi^{-} \oplus \phi, \\
H(6) & \text { hypermultiplet: } & 4(\mathbf{1}) \oplus\left(\mathbf{4}_{-}\right) & \rightarrow 4 \varphi^{\alpha} \oplus \chi^{-}, \\
V(6) & \text { vectormultiplet: } & (\mathbf{4}) \oplus\left(\mathbf{4}_{+}\right) & \rightarrow A_{\mu} \oplus \lambda^{+} .
\end{array}
$$

We explain below how the first situation arises in the cases of type IIA theory compactified on $K 3$ and heterotic $E_{8} \times E_{8}$ compactified on $T^{4}$, while the second situation arises in the case of heterotic $E_{8} \times E_{8}$ theory compactified on $K 3$.

\section{Type IIA on $K 3$}

Let us assume that $X^{4}$ is a $K 3$ surface and consider type IIA strings on $M^{1,5} \times X^{4}$, which yield an $\mathcal{N}=(1,1)$ supersymmetric theory. The NS-NS sector in $(2.1 .1)$ gives under $\mathfrak{s o}(4) \oplus \mathfrak{s o}(4)$ :

$$
\begin{aligned}
\mathbf{8}_{v} \otimes \mathbf{8}_{v} & =((\mathbf{4}, \mathbf{1}) \oplus(\mathbf{1}, \mathbf{4})) \otimes((\mathbf{4}, \mathbf{1}) \oplus(\mathbf{1}, \mathbf{4})) \\
& =\underbrace{(\mathbf{9}, \mathbf{1}) \oplus(\mathbf{4}, \mathbf{4}) \oplus(\mathbf{1}, \mathbf{1} \oplus \mathbf{9})}_{=\mathbf{3 5}} \oplus \underbrace{\left(\mathbf{3}_{+} \oplus \mathbf{3}_{-}, \mathbf{1}\right) \oplus(\mathbf{4}, \mathbf{4}) \oplus\left(\mathbf{1}, \mathbf{3}_{+} \oplus \mathbf{3}_{-}\right)}_{=\mathbf{2 8}} \oplus \underbrace{(\mathbf{1}, \mathbf{1})}_{=\mathbf{1}} .
\end{aligned}
$$

Here we use the decomposition of the 6 -dimensional representation $\Lambda^{2}\left(\mathbb{R}^{4}\right)$ of $\mathfrak{s o}(4)$ into the two irreducible representations $\Lambda_{ \pm}^{2}$ of dimension 3 .

At the level of fields this means that the graviton $g_{M N}$, which transforms in the $\mathbf{3 5}$ of $\mathfrak{s o}(8)$, decomposes into

$$
g_{M N} \longrightarrow g_{\mu \nu} \oplus g_{\mu i} \oplus g_{i j}
$$

where the six-dimensional graviton $g_{\mu \nu}$ gives the metric on the transversal directions of $M^{1,5}$ up to scaling (a spin 2 field), $g_{\mu i}$ are 1 -forms (spin 1 fields), and $g_{i j}$ are scalar fields (spin 0) on $M^{1,5}$. To count the dimension of the spaces of corresponding massless fields we need to consider each component as a section of a bundle on $K 3 . g_{\mu \nu}$ yields a scalar on $K 3$ which is 
automatically massless. Hence $g_{\mu \nu}$ gives one massless field in the $\mathbf{9}$ of $\mathfrak{s o}(4)$. Since $K 3$ has no closed one-forms, $g_{\mu i}$ generically can only contribute to the massive spectrum. To first order in perturbation theory the scalars $g_{i j}$ are the components of a Ricci-flat metric on $X$. The kernel of the linearized Einstein equation around $g_{i j}$ gives the corresponding massless fields. The dimension of this kernel is 58, the real dimension of the space of Einstein metrics on $K 3$.

Analogously, the $B$-field $B_{M N}$ decomposes into

$$
B_{M N} \longrightarrow B_{\mu \nu}^{+} \oplus B_{\mu \nu}^{-} \oplus B_{\mu i} \oplus B_{i j}^{+} \oplus B_{i j}^{-},
$$

where $B_{\mu \nu}^{+}$and $B_{\mu \nu}^{-}$are selfdual and respectively anti-selfdual 2 -forms, $B_{\mu i}$ a 1 -form and $B_{i j}$ scalar fields on $M^{1,5}$. The $B_{\mu \nu}^{ \pm}$give massless fields in the $\boldsymbol{3}_{ \pm}$of $\mathfrak{s o}(4)$, and the one-forms $B_{\mu i}$ become massive. The $B_{i j}^{ \pm}$give two-forms on $K 3$. The space of closed two-forms on $K 3$ is 22 dimensional, such that the $B_{i j}^{ \pm}$contribute a 22-dimensional space of massless scalars to the spectrum, called the $B$-field parameters.

The scalar dilaton in the $\mathbf{1}$ of $\mathfrak{s o}(8)$ descends to a scalar dilaton $\phi$ in the $\mathbf{1}$ of $\mathfrak{s o}(4)$.

In the R-NS sector, to decompose $\mathbf{5 6}_{+} \oplus \mathbf{8}_{-}=\Psi_{M}^{+} \oplus \Psi^{-}$it is again convenient to look at the complexifications:

$$
\begin{array}{ll}
\mathbf{5 6}_{+}^{\mathbb{C}} \longrightarrow\left(\mathbf{6}_{+}^{\mathbb{C}}, \mathbf{2}_{+}^{\mathbb{C}}\right) \oplus\left(\mathbf{6}_{-}^{\mathbb{C}}, \mathbf{2}_{-}^{\mathbb{C}}\right) \oplus\left(\mathbf{2}_{+}^{\mathbb{C}}, \mathbf{2}_{+}^{\mathbb{C}} \otimes \mathbf{4}^{\mathbb{C}}\right) \oplus\left(\mathbf{2}_{-}^{\mathbb{C}}, \mathbf{2}_{-}^{\mathbb{C}} \otimes \mathbf{4}^{\mathbb{C}}\right), & \mathbf{8}_{-}^{\mathbb{C}} \longrightarrow\left(\mathbf{2}_{-}^{\mathbb{C}}, \mathbf{2}_{+}^{\mathbb{C}}\right) \oplus\left(\mathbf{2}_{+}^{\mathbb{C}}, \mathbf{2}_{-}^{\mathbb{C}}\right), \\
\Psi_{M}^{+} \longrightarrow \psi_{\mu}^{+} \otimes \eta^{+} \oplus \psi_{\mu}^{-} \otimes \eta^{-} \oplus \quad \chi^{+} \otimes \psi_{i}^{+} \oplus \quad \chi^{-} \otimes \psi_{i}^{-}, & \Psi^{-} \longrightarrow \psi^{-} \otimes \eta^{+} \oplus \psi^{+} \otimes \eta^{-} .
\end{array}
$$

where we use $\mathbf{2}_{ \pm}^{\mathbb{C}} \otimes \mathbf{4}^{\mathbb{C}}=\mathbf{6}_{ \pm}^{\mathbb{C}} \oplus \mathbf{2}_{\mp}^{\mathbb{C}}$ as representations of $\mathfrak{s o}(4)$. In the real setting this corresponds

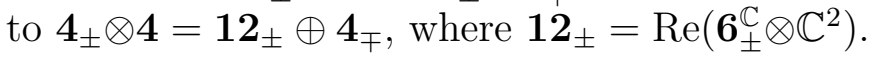

In the NS-R sector we similarly have:

$$
\begin{array}{ll}
\mathbf{5 6}_{-}^{\mathbb{C}} \longrightarrow\left(\mathbf{6}_{-}^{\mathbb{C}}, \mathbf{2}_{+}^{\mathbb{C}}\right) \oplus\left(\mathbf{6}_{+}^{\mathbb{C}}, \mathbf{2}_{-}^{\mathbb{C}}\right) \oplus\left(\mathbf{2}_{-}^{\mathbb{C}}, \mathbf{2}_{+}^{\mathbb{C}} \otimes \mathbf{4}^{\mathbb{C}}\right) \oplus\left(\mathbf{2}_{+}^{\mathbb{C}}, \mathbf{2}_{-}^{\mathbb{C}} \otimes \mathbf{4}^{\mathbb{C}}\right), & \mathbf{8}_{+}^{\mathbb{C}} \longrightarrow\left(\mathbf{2}_{+}^{\mathbb{C}}, \mathbf{2}_{+}^{\mathbb{C}}\right) \oplus\left(\mathbf{2}_{-}^{\mathbb{C}}, \mathbf{2}_{-}^{\mathbb{C}}\right), \\
\Psi_{M}^{-} \longrightarrow \psi_{\mu}^{-} \otimes \eta^{+} \oplus \psi_{\mu}^{+} \otimes \eta^{-} \oplus \quad \chi^{-} \otimes \psi_{i}^{+} \quad \oplus \quad \chi^{+} \otimes \psi_{i}^{-}, & \Psi^{+} \longrightarrow \psi^{+} \otimes \eta^{+} \oplus \psi^{-} \otimes \eta^{-} .
\end{array}
$$

The massless fields arise as spinors in the kernel of the respective Dirac operators on $K 3$, and the dimension of the space of massless spinors is given by the index of that Dirac operator. To obtain the number of independent gravitinos $\psi_{\mu}^{ \pm}$we use

$$
\text { ind } \not D=\int_{X} \widehat{A}\left(R_{0}\right)=2
$$

with $R_{0}$ the Riemann curvature tensor on $X$. This means that the space of solutions to the massless field equation $\not D \psi=0$ which cannot become massive has complex dimension 2. From (2.2.1), a pair of two complex spinors in the kernel of $\not D$ gives one positive chirality RaritaSchwinger gravitino. Hence we get one positive chirality Rarita-Schwinger gravitino $\psi_{\mu}^{+}$from $\Psi_{M}^{+}$and one negative chirality Rarita-Schwinger gravitino $\psi_{\mu}^{-}$from $\Psi_{M}^{-}$, respectively. The same index calculation also shows that the ten-dimensional dilatinos $\Psi^{\mp}$ contribute six-dimensional dilatinos $\psi^{\mp}$. The fermions $\chi^{ \pm}$coming from the ten-dimensional gravitinos $\Psi_{\mu}^{ \pm}$are governed by the index of the Rarita-Schwinger Dirac operator $\widetilde{D}_{R S}$ on sections of $S^{ \pm} \otimes T^{*} X$, where $S^{ \pm}$ denote the spinor bundles on $X$. By (B.9) we have ind $\left(\widetilde{D}_{R S}\right)=-40$, amounting to 20 negative chirality spinors $\chi^{-}$coming from $\Psi_{M}^{+}$and 20 positive chirality spinors $\chi^{+}$coming from $\Psi_{M}^{-}$. 
In the R-R sector we finally have:

$$
\begin{gathered}
\mathbf{5 6}_{v} \longrightarrow(\mathbf{4}, \mathbf{1}) \oplus\left(\mathbf{3}_{+} \oplus \mathbf{3}_{-}, \mathbf{4}\right) \oplus\left(\mathbf{4}, \mathbf{3}_{+} \oplus \mathbf{3}_{-}\right) \oplus(\mathbf{1}, \mathbf{4}), \quad \mathbf{8}_{v} \longrightarrow(\mathbf{4}, \mathbf{1}) \oplus(\mathbf{1}, \mathbf{4}), \\
C_{M N P} \longrightarrow C_{\mu \nu \rho} \oplus \quad C_{\mu \nu i} \oplus \quad C_{\mu i j} \oplus C_{i j k}, \quad C_{M} \longrightarrow C_{\mu} \oplus C_{i} .
\end{gathered}
$$

The space-time two-forms $C_{\mu \nu i}$ and the zero-forms $C_{i j k}, C_{i}$ give one-forms and three-forms on $K 3$ respectively; generically they are massive since there are no harmonic one-forms or threeforms on $K 3$. Massless fields arise from the three-form $C_{\mu \nu \rho}$ and from the one-forms $C_{\mu i j}, C_{\mu}$, which are zero-forms and two-forms on $K 3$, respectively. Since the spaces of closed zero- and two-forms on $K 3$ are one- and 22-dimensional, in total we get a 24-dimensional space of massless fields, all of which transform in the 4 of $\mathfrak{s o}(4)$. In summary,

Proposition 2.1. The massless spectrum of type IIA strings compactified to six dimensions on an internal $K 3$ surface contains 1 graviton $g_{\mu \nu}$ in the (9), 81 (linearly independent) scalars $g_{i j}, B_{i j}, \phi$ in the $(\mathbf{1})$, a selfdual and an anti-selfdual $B_{\mu \nu}^{ \pm}$in the $\left(\mathbf{3}_{ \pm}\right), 2$ gravitinos $\psi_{\mu}^{ \pm}$in the $\left(\mathbf{1 2}_{ \pm}\right), 2$ dilatinos $\psi^{\mp}$ in the $\left(\mathbf{4}_{\mp}\right), 40$ fermions $\chi^{ \pm}$in the $\left(\mathbf{4}_{ \pm}\right)$, and 24 vectors $C_{\mu \nu \rho}, C_{\mu i j}, C_{\mu}$ in the (4) of $\mathfrak{s o}(4)$. They are grouped into supermultiplets according to (2.2.3), where for definiteness we set $C:=\left(C_{\mu \nu \rho}, C_{\mu i j}^{(2,0)}, C_{\mu i j}^{(0,2)}, C_{\mu}\right)$ with the superscripts indicating the Dolbeault grading of the cohomology of $K 3$ :

$1 \times($ supergravity multiplet) :

$$
\begin{gathered}
(\mathbf{9}) \oplus\left(\mathbf{1 2}_{+}\right) \oplus\left(\mathbf{1 2}_{-}\right) \oplus 4(\mathbf{4}) \oplus\left(\mathbf{3}_{+}\right) \oplus\left(\mathbf{3}_{-}\right) \oplus\left(\mathbf{4}_{+}\right) \oplus\left(\mathbf{4}_{-}\right) \oplus(\mathbf{1}) \\
\left(g_{\mu \nu}\right) \oplus \psi_{\mu}^{+} \oplus \psi_{\mu}^{-} \oplus C \oplus B_{\mu \nu}^{+} \oplus B_{\mu \nu}^{-} \oplus \psi^{+} \oplus \psi^{-} \oplus \phi
\end{gathered}
$$

$20 \times($ matter multiplet) :

$$
\begin{gathered}
80(\mathbf{1}) \oplus 20(\mathbf{4}) \oplus 20\left(\left(\mathbf{4}_{+}\right) \oplus\left(\mathbf{4}_{-}\right)\right) \\
\left(g_{i j}, B_{i j}\right) \oplus C_{i j}^{(1,1)} \oplus \quad \chi^{+} \oplus \chi^{-} .
\end{gathered}
$$

\section{Heterotic $E_{8} \times E_{8}$ strings on $K 3$}

We now follow [Wal88, p. 379], changing the chiralities in ten dimensions to satisfy the conventions in [GSW85] and adding in further details.

We again assume that $X^{4}$ is a $K 3$ surface and consider $G_{10}=E_{8} \times E_{8}$ heterotic strings on $M^{1,5} \times X^{4}$. This means that $M^{1,5} \times X^{4}$ carries a gauge bundle $\mathcal{E}$ with holonomy $G_{10}$. The field $A_{M}$ in the Yang-Mills hypermultiplet $Y(10)$ of (2.1.3) yields its connection. To compactify we also need to assume that the holonomy of $\mathcal{E}$ decomposes into a product $H \times K \subset G_{10}$ with $H$ the holonomy of $\mathcal{E}$ viewed as a bundle on $M^{1,5}$ and $K$ the holonomy of $\mathcal{E}$ viewed as a bundle on $X^{4}$. This is detailed and used below (2.2.6). Viewed as a bundle on $X$, for reasons of consistency the connection of $\mathcal{E}$ is Hermitian-Yang-Mills, where the Donaldson-Uhlenbeck-Yau theorem [Don85, UY86] states that equivalently $\mathcal{E}$ is semi-stable. Since $X$ is a Kähler manifold of complex dimension 2, Hermitian-Yang-Mills connections are precisely the anti-selfdual (ASD) connections.

We have listed the massless ten-dimensional multiplets of heterotic strings in (2.2.4), so a general theory has massless spectrum

$$
R(6) \oplus n_{T} T(6) \oplus n_{H} H(6) \oplus n_{V} V(6),
$$


where we need to determine $n_{T}, n_{H}, n_{V}$.

If we are compactifying a theory which possesses a Lorentz-invariant action, then self-dual and anti-self dual two-forms $B_{\mu \nu}^{ \pm}$are paired up, hence there is only one tensormultiplet, $n_{T}=1$ [DHVW85, DHVW86]. This is the case we focus on first. On the other hand, a general theory need not arise from compactification, and $n_{T}>1$ is allowed.

The supergravity multiplet $R(10)$ of (2.1.3) agrees with the NS-NS plus the R-NS sector of type IIA. Hence to compactify to six dimensions we can use the previous results obtaining as massless fields: $g_{\mu \nu}$, the graviton in the (9), 81 scalars $g_{i j}, B_{i j}, \phi$ in the $(\mathbf{1})$, selfdual and anti-selfdual two-forms $B_{\mu \nu}^{ \pm}$in the $\left(\mathbf{3}_{ \pm}\right)$, a gravitino $\psi_{\mu}^{+}$in the $(\mathbf{1 2}+)$, a dilatino $\psi^{-}$in the $\left(\mathbf{4}_{-}\right)$, and 20 fermions $\chi^{-}$in the $\left(\mathbf{4}_{-}\right)$. A quick glance at (2.2.4) reveals that the $\psi^{-}, \chi^{-}$can either belong to a tensor or a hypermultiplet. However, we know that we need to produce precisely one tensormultiplet, so we can safely assume that $\psi^{-}$resides in the tensormultiplet, while the $\chi^{-}$live in hypermultiplets. Altogether we have listed the content of the following multiplets:

$$
R(10) \longrightarrow R(6) \oplus T(6) \oplus 20 H(6) .
$$

To determine $n_{H}, n_{V}$ in (2.2.5), note that the chirality of the fields $\chi^{-}, \lambda^{+}$respectively distinguishes hypermultiplets from vectormultiplets, the only two types of multiplets that are left for $Y(10)$ to contribute to. Hence it suffices to consider the reduction of the only fermionic field $\Lambda^{+}$in the super-Yang-Mills multiplet $Y(10)$ of (2.1.3). The gaugino $\Lambda^{+}$is a positive spinor on $M^{1,9}$ with values in the adjoint representation of the gauge group $G_{10}=E_{8} \times E_{8}$ of the tendimensional theory. On reducing from ten to six dimensions the gauge group is broken to some subgroup $H=H^{1} \times H^{2} \subset E_{8} \times E_{8}$ with $H^{i} \subset E_{8}$, while the components $A_{i}$ of the gauge field $A$ with $K 3$ indices, the Higgs bosons, acquire expectation values in $K=K^{1} \times K^{2} \subset E_{8} \times E_{8}$, with $K^{i} \subset E_{8}$ the maximal simple subgroup of the centralizer of $H^{i}$. This means that the gauge bundle $\mathcal{E}$, which we always view as a sum of two $E_{8}$ bundles $\mathcal{E}^{i}$, gives a $K$-principal bundle on $K 3$ with curvature taking values in $\operatorname{adj}(\mathrm{K})$.

The adjoint representation of $E_{8}$ now decomposes under each $H^{i} \times K^{i}$ as

$$
\operatorname{adj}\left(E_{8}\right)=\bigoplus_{a \in A^{i}} L_{a}^{i} \otimes Q_{a}^{i}=\left(\operatorname{adj}\left(H^{i}\right) \otimes \mathbf{1}\right) \oplus \bigoplus_{a \in A_{\text {matter }}}\left(L_{a}^{i} \otimes Q_{a}^{i}\right),
$$

where $L_{a}^{i}$ and $Q_{a}^{i}$ are representations of $H^{i}$ and $K^{i}$. In particular,

$$
\begin{aligned}
\sum_{a \in A_{\text {matter }}^{i}} \operatorname{dim} Q_{a}^{i} \cdot \operatorname{dim} L_{a}^{i} & =\sum_{a \in A^{i}} \operatorname{dim} Q_{a}^{i} \cdot \operatorname{dim} L_{a}^{i}-\operatorname{dim}\left(H^{i}\right) \\
& =\operatorname{dim}\left(\operatorname{adj}\left(\mathrm{E}_{8}\right)\right)-\operatorname{dim}\left(H^{i}\right)=248-\operatorname{dim}\left(H^{i}\right) .
\end{aligned}
$$

Representations with labels in $A_{\text {matter }}^{i}$ comprise the so-called matter multiplets. Here $\mathbf{1} \otimes \operatorname{adj}\left(\mathrm{K}^{\mathrm{i}}\right)$ gives the neutral matter multiplets, and all other $L_{a}^{i} \otimes Q_{a}^{i}$ with $a \in A_{\text {matter }}^{i}$ give charged matter multiplets. We have

$$
\Lambda^{+}=\sum_{i, a \in A^{i}}\left(\lambda_{a, i}^{+} \otimes \eta_{a, i}^{+}+\lambda_{a, i}^{-} \otimes \eta_{a, i}^{-}\right)
$$

where $\lambda_{a, i}^{ \pm}$is a section of $S_{M}^{ \pm} \otimes L_{a}^{i}$, and $\eta_{a, i}^{ \pm}$is a section of $S^{ \pm} \otimes Q_{a}^{i}$, with $S_{M}^{ \pm}, S^{ \pm}$the spinor bundles on $M$ and $X$, respectively. To determine the net number of massless fields we need to 
calculate the index of a Dirac operator $\not_{Q}: S^{+} \otimes \mathcal{E}_{Q}^{i} \rightarrow S^{-} \otimes \mathcal{E}_{Q}^{i}$ on $K 3$, twisted by a $K^{i}$-bundle $\mathcal{E}_{Q}^{i}$ corresponding to the representation $Q$ of $K^{i}$. Since

$$
\text { ind }\left(\not D_{Q}\right)=- \text { ind }\left(\not D_{Q^{*}}\right) \text {, }
$$

the index vanishes if $Q$ is a real (or pseudoreal) representation. For complex representations $Q$ we have

$$
\operatorname{ind}\left(\not D_{Q}\right)=\int_{X} \widehat{A}\left(R_{0}\right) \operatorname{ch}(Q)
$$

where $R_{0}$ is the Riemannian curvature of the $K 3$ surface and $\operatorname{ch}(Q)$ is a form in the induced curvature $F_{0}$ of the associated bundle $\mathcal{E}_{Q}^{i}$. See Appendix B for further details on characteristic classes.

In general for a simple Lie group $G$ with Lie algebra $\mathfrak{g}$, for any $G$-bundle $\mathcal{E}_{Q}^{i}$ corresponding to a representation $Q$ of $G$ we introduce the first Pontrjagin class $p_{1}\left(\mathcal{E}_{Q}^{i}\right)$ :

$$
p_{1}\left(\mathcal{E}_{Q}^{i}\right):=-\frac{1}{8 \pi^{2}} \int_{X} \operatorname{Trace}_{Q}\left(F_{0}^{2}\right) .
$$

Then expansion of $\operatorname{ch}(Q)$ yields

$$
\operatorname{ind}\left(D_{Q}\right)=\operatorname{dim} Q \int_{X} \widehat{A}\left(R_{0}\right)-\frac{1}{8 \pi^{2}} \int_{X} \operatorname{Trace}_{Q}\left(F_{0}^{2}\right) \stackrel{(B .8)}{=} 2 \operatorname{dim} Q+p_{1}\left(\mathcal{E}_{Q}^{i}\right) .
$$

Next, following [AHS78] for any $G$-bundle on $X$ the instanton number is given by

$$
k:=\frac{1}{8 \pi^{2}} \frac{1}{2 c_{2}(G)} \int_{X} \operatorname{Trace}_{\mathfrak{g}}\left(F_{0}^{2}\right),
$$

where $c_{2}(G)$ is the dual Coxeter number of $G$. It is important to note that the instanton number is a topological invariant of the bundle. If the holonomy is a proper subgroup of $G$, then the instanton number is also equal to the expression on the right hand side of (2.2.12) where $G$ is replaced by the holonomy group and $\mathfrak{g}$ by its Lie algebra. Hence the expression (2.2.12) could be used as the definition of the dual Coxeter number $c_{2}(G)$. Now with $\mathfrak{k}^{i}$ the Lie algebra of $K^{i}$, the instanton number of the $E_{8}$-bundle $\mathcal{E}^{i}$ is

$$
k^{i}=\frac{1}{8 \pi^{2}} \frac{1}{2 c_{2}\left(K^{i}\right)} \int_{X} \operatorname{Trace}_{\mathfrak{k}^{i}}\left(F_{0}^{2}\right)=\frac{1}{8 \pi^{2}} \frac{1}{2 c_{2}\left(E_{8}\right)} \int_{X} \operatorname{Trace}_{\mathfrak{e}_{8}}\left(F_{0}^{2}\right) .
$$

Since $c_{2}\left(E_{8}\right)=30$ and by $(2.2 .10)$ we thus have

$$
p_{1}\left(\mathcal{E}^{i}\right)=-60 k^{i} .
$$

Using the so-called index ind $(Q)$ of the representation $Q$, which is defined by

$$
\forall Y, Z \in \mathfrak{k}^{i}: \quad \operatorname{Trace}_{Q}(Y \circ Z)=\frac{\text { ind }(Q)}{c_{2}\left(K^{i}\right)} \operatorname{Trace}_{\mathfrak{k}^{i}}(Y \circ Z),
$$

we moreover get

$$
p_{1}\left(\mathcal{E}_{Q}^{i}\right)=-2 k^{i} \text { ind }(Q) \text {. }
$$


Hence (2.2.11) yields

$$
\operatorname{ind}\left(\not D_{Q}\right)=2 \operatorname{dim}(Q)-2 k^{i} \text { ind }(Q) \text {. }
$$

This number is related ${ }^{3}$ to the dimension of the moduli space of irreducible ASD connections on $\mathcal{E}_{Q}^{i}$ : The moduli space is either empty or has the hyperkähler dimension

$$
k^{i} \text { ind }(Q)-\operatorname{dim}(Q) .
$$

The proof is essentially identical to the proof for the analogous theorem [AHS78, Thm. 6.1], with the only modification that now we work with ASD connections on a vector bundle $\mathcal{E}_{Q}^{i}$. In order to complete the counting of the various multiplets below, we note

$$
\begin{aligned}
& p_{1}\left(\mathcal{E}^{i}\right) \stackrel{(2.2 .10)}{=}-\frac{1}{8 \pi^{2}} \int_{K 3} \operatorname{Trace}_{\mathfrak{e}_{8}}\left(F_{0}^{2}\right) \\
& \stackrel{(2.2 .7)}{=}-\sum_{a \in A^{i}} \operatorname{dim} L_{a}^{i} \cdot \frac{1}{8 \pi^{2}} \int_{K 3} \operatorname{Trace}_{Q_{a}^{i}}\left(F_{0}^{2}\right) \stackrel{(2.2 .14)}{=}-2 k^{i} \sum_{a \in A^{i}} \operatorname{dim} L_{a}^{i} \cdot \operatorname{ind}\left(Q_{a}^{i}\right) \text {. }
\end{aligned}
$$

Since $A_{\text {matter }}^{i}$ differs from $A^{i}$ only by $L_{a}^{i}=\operatorname{adj}\left(\mathrm{H}^{\mathrm{i}}\right)$, with $Q_{a}^{i}=\mathbf{1}$ and ind $(\mathbf{1})=0$ this gives

$$
k^{i} \sum_{a \in A_{\text {matter }}^{i}} \operatorname{dim} L_{a}^{i} \cdot \operatorname{ind}\left(Q_{a}^{i}\right)=k^{i} \sum_{a \in A^{i}} \operatorname{dim} L_{a}^{i} \cdot \operatorname{ind}\left(Q_{a}^{i}\right)=-\frac{p_{1}\left(\mathcal{E}^{i}\right)}{2} \stackrel{(2.2 .13)}{=} 30 k^{i} .
$$

Finally returning to the reduction of the ten-dimensional gaugino $\Lambda^{+}$under compactification to six dimensions in (2.2.9), we set

$$
N\left(L_{a}^{i}\right):=\frac{1}{2} \operatorname{ind}\left(\not Q_{Q_{a}^{i}}\right) \stackrel{(2.2 .15)}{=} \operatorname{dim} Q_{a}^{i}-k^{i} \cdot \operatorname{ind}\left(Q_{a}^{i}\right),
$$

where we have taken into account that pairs of spinors have to be considered in order to count Weyl spinors. As discussed at the beginning of Section 2.2, if $N\left(L_{a}^{i}\right)$ is positive, then we have positive chirality spinors from the Minkowski point of view, i.e. using (2.2.4) there are $N\left(L_{a}^{i}\right)$ vectormultiplets in the representation $L_{a}^{i}$ of the unbroken gauge group. If $N\left(L_{a}^{i}\right)$ is negative, then accordingly we have $\left(-N\left(L_{a}^{i}\right)\right)$ hypermultiplets in the representation $L_{a}^{i}$ of the unbroken gauge group. In particular, $N\left(\operatorname{adj}\left(\mathrm{H}^{\mathrm{i}}\right)\right)=1$. For all other labels $a \in A_{\text {matter }}^{i}$, by $(2.2 .16)$ we know that $\left(-N\left(L_{a}^{i}\right)\right)$ gives the dimension of the moduli space of ASD connections on $\mathcal{E}_{Q_{a}^{i}}^{i}$. This number must be non-negative in order for such a theory with unbroken gauge group $H=H^{1} \times H^{2}$ to exist. Hence

$$
Y(10) \longrightarrow \sum_{i} \operatorname{dim}\left(H^{i}\right) \cdot V(6) \oplus \sum_{i, a \in A_{\text {matter }}^{i}}\left(-N\left(L_{a}^{i}\right)\right) \operatorname{dim} L_{a}^{i} \cdot H(6),
$$

where

$$
\begin{gathered}
\sum_{a \in A_{\text {matter }}^{i}}\left(-N\left(L_{a}^{i}\right)\right) \operatorname{dim} L_{a}^{i} \stackrel{(2.2 .18)}{=} k^{i} \cdot \sum_{a \in A_{\text {matter }}^{i}} \operatorname{ind}\left(Q_{a}^{i}\right) \cdot \operatorname{dim} L_{a}^{i}-\sum_{a \in A_{\text {matter }}^{i}} \operatorname{dim} Q_{a}^{i} \cdot \operatorname{dim} L_{a}^{i} \\
\stackrel{(2.2 .17),(2.2 .8)}{=} 30 k^{i}-248+\operatorname{dim}\left(H^{i}\right) .
\end{gathered}
$$

\footnotetext{
${ }^{3}$ This and the derivation of (2.2.19) following Proposition 2.2 is present between the lines in the physics literature, but we could not find it phrased out explicitly.
} 
Remark. As already pointed out, for a theory as above to exist we must have

$$
N\left(L_{a}^{i}\right)=\operatorname{dim} Q_{a}^{i}-k^{i} \cdot \text { ind }\left(Q_{a}^{i}\right) \leq 0, \quad \text { for all } a \in A_{\text {matter }}^{i} .
$$

These inequalities give lower bounds on the instanton numbers $k^{1}, k^{2}$.

So far, we have worked with smooth bundles $\mathcal{E}^{i}$ on smooth $K 3$ surfaces. However, interesting new structures arise when these bundles degenerate to pointlike instantons, i.e. when the curvature of $\mathcal{E}^{i}$ acquires singularities in the form of Dirac delta distributions. In [DK90, Definition 4.4.1] the resulting connections on $\mathcal{E}^{i}$ are called ideal ASD connections. In this situation the Pontrjagin classes (2.2.10) and the instanton numbers (2.2.12) are defined in terms of the smooth part $F_{0}$ of the curvature. According to [SW96, Wit96b] non-perturbative strong coupling singularities occur when instantons become pointlike, associated to tensionless strings. The process is accompanied by the emergence of an additional tensormultiplet for each pointlike instanton along with an additional neutral hypermultiplet contributing scalars which account for the location of the instanton on the $K 3$ surface. The scalar in the additional tensormultiplet is believed to give the parameter of a non-classical phase transition. Altogether the contributions from the Yang-Mills sector amount to

$$
\begin{aligned}
l T(6) & \oplus \quad\left(l-\sum_{i, a \in A_{\text {matter }}^{i}} N\left(L_{a}^{i}\right) \operatorname{dim} L_{a}^{i}\right) H(6) \oplus \sum_{i} \operatorname{dim}\left(H^{i}\right) V(6) \\
\stackrel{(2.2 .20)}{=} & l T(6) \oplus\left(30\left(k^{1}+k^{2}\right)-496+\operatorname{dim}(H)+l\right) H(6) \oplus \operatorname{dim}(H) V(6)
\end{aligned}
$$

if there are $l$ distinct pointlike instantons. According to what was said after (2.2.8), of the hypermultiplets, $\left(k^{1} c_{2}\left(K^{1}\right)+k^{2} c_{2}\left(K^{2}\right)-\operatorname{dim} K+l\right)$ are neutral.

One constraint to our theory coming from the Green-Schwarz mechanism in ten dimensions (see (3.2.1) with $1 / \alpha=-c_{2}\left(E_{8}\right)=-30$ as argued below (3.2.2)) is

$$
\int_{K 3}\left(\operatorname{tr}\left(\mathrm{R}_{0}^{2}\right)-\frac{1}{30} \operatorname{Trace}_{\mathfrak{e}_{8} \oplus \mathfrak{e}_{8}}\left(\check{\mathrm{F}}_{0}^{2}\right)\right)=0,
$$

where $\check{F}_{0}$ denotes the total curvature of $\mathcal{E}$, i.e. $F_{0}$ plus contributions of Dirac delta distributions from pointlike instantons. With the correct normalization of the Dirac delta distribution one gets

$$
\begin{aligned}
24=\chi(K 3) & =\frac{1}{16 \pi^{2}} \int_{X} \operatorname{tr}\left(R_{0}^{2}\right)=\frac{1}{60} \frac{1}{8 \pi^{2}} \int_{X} \operatorname{Trace}_{\mathfrak{e}_{8} \oplus \mathfrak{e}_{8}}\left(F_{0}^{2}\right)+l \\
\stackrel{(2.2 .10)}{=} & -\frac{1}{60} p_{1}\left(\mathcal{E}^{1}\right)-\frac{1}{60} p_{1}\left(\mathcal{E}^{2}\right)+l \stackrel{(2.2 .13)}{=} k^{1}+k^{2}+l .
\end{aligned}
$$

Hence (2.2.6) and (2.2.21) give

Proposition 2.2. Consider a theory with unbroken gauge group $H$ which is obtained by compactification to six dimensions on a smooth internal $K 3$ surface from a ten-dimensional $E_{8} \times E_{8}$ heterotic string theory. Moreover, assume that the bundle on $K 3$ has degenerated to receive $l$ distinct pointlike instantons. Then the massless spectrum is given by

$$
R(6) \oplus(l+1) T(6) \oplus(244+\operatorname{dim} H-29 l) H(6) \oplus \operatorname{dim}(H) V(6) .
$$


The $n_{H}$ hypermultiplets receive 20 contributions that give moduli of the $K 3$ surface. The bundle $\mathcal{E}$ on $K 3$ decomposes into two $E_{8}$-bundles $\mathcal{E}^{1}, \mathcal{E}^{2}$ with instanton numbers $k^{1}, k^{2}$ such that $k^{1}+$ $k^{2}+l=24$, and with holonomy groups $K^{1}, K^{2}$, where $K^{1} \times K^{2} \subset E_{8} \times E_{8}$ is the maximal simple subgroup of the centralizer of $H$. Then with $c_{2}\left(K^{i}\right)$ denoting the respective dual Coxeter numbers, further

$$
k^{1} c_{2}\left(K^{1}\right)+k^{2} c_{2}\left(K^{2}\right)-\operatorname{dim} K+l
$$

of the hypermultiplets are neutral and give moduli of the bundle $\mathcal{E}$ on $K 3$.

Remark. To clear notations, we introduce $n_{H}^{0}$ and $n_{H}^{c h}$, the numbers of neutral and charged hypermultiplets, respectively, so that generically,

$$
n_{H}=n_{H}^{0}+n_{H}^{c h}, \quad n_{H}^{0}=20+k^{1} c_{2}\left(K^{1}\right)+k^{2} c_{2}\left(K^{2}\right)-\operatorname{dim} K+l .
$$

If $K$ contains factors that are non-simply laced Lie groups then the decomposition $n_{H}=$ $n_{H}^{0}+n_{H}^{c h}$ into uncharged and charged matter is a little more subtle: In (2.2.7) the corresponding summands $L_{a}^{i} \otimes Q_{a}^{i}$ with non-trivial $L_{a}^{i}$ and $Q_{a}^{i}$ can have non-zero subspaces that are uncharged under the gauge group. This increases $n_{H}^{0}$ and accordingly decreases $n_{H}^{c h}$ compared to the above formula. We will briefly discuss this effect in Section 5.2.2 below. See also Section 3.3.5 for an example.

\section{Heterotic $E_{8} \times E_{8}$ strings on $T^{4}$}

Let us now consider heterotic $G_{10}=E_{8} \times E_{8}$ strings compactified to $M^{1,5} \times X^{4}$ where $X^{4}$ is a real four-torus. As opposed to the case where $X^{4}$ is a $K 3$ surface these theories enjoy enhanced supersymmetry since the holonomy of the torus is trivial, and hence all components of the supercharges in the ten-dimensional theory yield components of supercharges of the compactified theory, see e.g. [Asp97, p. 38]. In this situation massless particles of opposite chirality do not pair up to become massive. However, since all fields give flat sections of the relevant bundles on the torus $X^{4}$, starting from the massless spectrum (2.1.3) of the tendimensional theory we can decompose all representations with respect to the space-time $\mathfrak{s o}(4)$ as before and take the results at face value without any index calculations. The gauge group is generically broken to $H_{\text {gen }}=U(1)^{16}$. Since $\operatorname{dim} H_{\text {gen }}=16$, and using the decompositions obtained previously, we generically have

$$
\begin{array}{llll}
\text { 35: } & g_{M N} & \rightarrow g_{\mu \nu} \oplus g_{\mu i} \oplus g_{i j} & \text { in } 1(\mathbf{9}) \oplus 4(\mathbf{4}) \oplus 10(\mathbf{1}), \\
\mathbf{2 8 :} & B_{M N} & \rightarrow B_{\mu \nu}^{+} \oplus B_{\mu \nu}^{-} \oplus B_{\mu i} \oplus B_{i j} & \text { in } 1\left(\mathbf{3}_{+}\right) \oplus 1\left(\mathbf{3}_{-}\right) \oplus 4(\mathbf{4}) \oplus 6(\mathbf{1}), \\
\mathbf{1 :} & \phi & \rightarrow \phi & \text { in } 1(\mathbf{1}), \\
\mathbf{5 6}_{+}: & \Psi_{M}^{+} & \rightarrow \psi_{\mu}^{+} \oplus \psi_{\mu}^{-} \oplus \chi^{+} \oplus \chi^{-} & \text {in }\left(\mathbf{1 2}_{+}\right) \oplus\left(\mathbf{1 2}_{-}\right) \oplus 4\left(\left(\mathbf{4}_{+}\right) \oplus\left(\mathbf{4}_{-}\right)\right), \\
\mathbf{8}_{-}: & \Psi^{-} & \rightarrow \psi^{-} \oplus \psi^{+} & \text {in }\left(\mathbf{4}_{+}\right) \oplus\left(\mathbf{4}_{-}\right), \\
\mathbf{8}_{v} \otimes \operatorname{Ad}\left(G_{10}\right): & A_{M} & \rightarrow A_{\mu} \oplus A_{i} & \text { in } 16((\mathbf{4}) \oplus 4(\mathbf{1})), \\
\mathbf{8}_{+} \otimes \operatorname{Ad}\left(G_{10}\right): & \Lambda^{+} & \rightarrow \lambda^{+} \oplus \lambda^{-} & \text {in } 16\left(\left(\mathbf{4}_{+}\right) \oplus\left(\mathbf{4}_{-}\right)\right) .
\end{array}
$$

Altogether we find one graviton $g_{\mu \nu}$ in the (9), 24 (linearly independent) vectors $g_{\mu i}, B_{\mu i}, A_{\mu}$ in the (4), 81 scalars $g_{i j}, B_{i j}, \phi, A_{i}$ in the (1), a selfdual and an anti-selfdual two-form $B_{\mu \nu}^{ \pm}$in the $\left(\mathbf{3}_{ \pm}\right), 2$ gravitinos $\psi_{\mu}^{ \pm}$in the $\left(\mathbf{1 2}_{ \pm}\right), 40$ fermions $\chi^{ \pm}, \lambda^{ \pm}$in the $\left(\mathbf{4}_{ \pm}\right)$, and 2 dilatinos $\psi^{\mp}$ in the $\left(\mathbf{4}_{\mp}\right)$ of $\mathfrak{s o}(4)$. Comparison with (2.2.3) and Proposition 2.1 shows 
Proposition 2.3. The massless spectrum of heterotic $E_{8} \times E_{8}$ strings compactified to six dimensions on an internal four-torus generically consists of 1 supergravity and 20 matter multiplets in (2.2.3) and agrees with the massless spectrum of type IIA strings compactified to six dimensions on an internal K3 surface by Proposition 2.1.

The agreement of the generic massless spectra of heterotic $E_{8} \times E_{8}$ strings on $T^{4}$ and type IIA strings on $K 3$ nowadays is believed not to be a coincidence. We will address this issue in Section 4.

\subsubsection{Compactification to $D=4$}

Consider now superstring theories on $M^{1,3} \times X^{6}$. We will need to decompose our representations of $\mathfrak{s o}(8)$ under $\mathfrak{s o}(2) \oplus \mathfrak{s o}(6)$, where for $\mathfrak{s o}(2)$ we denote the two-dimensional tensor, vector, and spin $\frac{3}{2}$ representations by $\boldsymbol{2}_{t}, \mathbf{2}_{v}, \mathbf{2}_{s}$, respectively, all of which are equivalent. There also exists a "spinor" in two dimensions, which transforms trivially under $\mathfrak{s o}(2)$, denoted $\mathbf{1}_{s}$. The possible massless supermultiplets for $\mathcal{N}=2$ supersymmetry are then

$$
\begin{array}{lll}
R(4) & \text { supergravity multiplet: } & \left(\mathbf{2}_{t}\right) \oplus 2\left(\mathbf{2}_{s}\right) \oplus\left(\mathbf{2}_{v}\right), \\
H(4) & \text { hypermultiplet: } & 4\left(\mathbf{1}_{s}\right) \oplus 4(\mathbf{1}), \\
V(4) \text { vectormultiplet: } & \left(\mathbf{2}_{v}\right) \oplus 4\left(\mathbf{1}_{s}\right) \oplus 2(\mathbf{1}) .
\end{array}
$$

For our discussion it hence suffices to consider the bosonic parts of the massless spectra: These already uniquely determine the numbers of independent supermultiplets of each type. In what follows we describe this analysis for type IIA compactified on a Calabi-Yau 3-fold, and for heterotic $E_{8} \times E_{8}$ compactified on $T^{2} \times K 3$.

\section{Type IIA on a Calabi-Yau 3-fold}

Assume that $X^{6}$ is a Calabi-Yau 3-fold with full SU(3) holonomy, i.e. with Betti numbers

$$
b_{0}=b_{6}=1, \quad b_{1}=b_{5}=0, \quad b_{2}=b_{4}=h^{1,1}(X), \quad b_{3}=2\left(h^{1,2}(X)+1\right) .
$$

To obtain the massless spectrum of type IIA strings compactified to four dimensions with internal $X$ we decompose the bosonic massless fields of the ten-dimensional theory in the NSNS and the R-R sectors as given in (2.1.1) as before:

$$
\begin{array}{llll}
\text { 35: } & g_{M N} \rightarrow g_{\mu \nu} \oplus g_{\mu i} \oplus g_{i j} & \text { in } 1\left(\mathbf{2}_{t}\right) \oplus 6\left(\mathbf{2}_{v}\right) \oplus 21(\mathbf{1}), \\
\mathbf{2 8 :} & B_{M N} \rightarrow B_{\mu \nu} \oplus B_{\mu i} \oplus B_{i j} & \text { in } 1(\mathbf{1}) \oplus 6\left(\mathbf{2}_{v}\right) \oplus 15(\mathbf{1}), \\
\mathbf{1 :} & \phi & \rightarrow \phi & \text { in } 1(\mathbf{1}), \\
\mathbf{5 6}_{v}: & C_{M N P} \rightarrow C_{\mu \nu i} \oplus C_{\mu i j} \oplus C_{i j k} & \text { in } 6(\mathbf{1}) \oplus 15\left(\mathbf{2}_{v}\right) \oplus 20(\mathbf{1}), \\
\mathbf{8}_{v}: & C_{M} \rightarrow C_{\mu} \oplus C_{i} & \text { in } 1\left(\mathbf{2}_{v}\right) \oplus 6(\mathbf{1}) .
\end{array}
$$

Since $X$ carries no closed one-forms, $g_{\mu i}, B_{\mu i}, C_{\mu \nu i}$ and $C_{i}$ become massive. $g_{\mu \nu}$ contributes the graviton in the $\mathbf{2}_{t}$ to the massless spectrum. The $g_{i j}$ contribute massless scalars, according to the Ricci-flat deformations of this metric on $X$. There are two types of deformations of this Ricci-flat metric: (1) those corresponding to deformations of a chosen complex structure 
and giving in total $2 h^{1,2}(X)$ parameters; (2) those corresponding to deformations of a chosen Kähler structure and giving a total of $h^{1,1}(X)$ parameters. This analysis uses the fact that the moduli space of Calabi-Yau 3-folds locally splits into the product of Kähler and complex structure deformations. In total, one gets $h^{1,1}(X)+2 h^{1,2}(X)$ independent scalars coming from $g_{i j}$. Moreover, $a:=B_{\mu \nu}$ contributes a massless scalar, known as the axion, while $B_{i j}$ gives $b_{2}=h^{1,1}(X)$ massless scalars. The dilaton $\phi$ is a scalar as always. $C_{\mu i j}$ gives $b_{2}=h^{1,1}(X)$ massless fields in the $\mathbf{2}_{v}$, while $C_{i j k}$ gives $b_{3}=2\left(h^{1,2}(X)+1\right)$ additional massless scalars. Finally, $C_{\mu}$ contributes one massless vector in the $\boldsymbol{2}_{v}$, known as the graviphoton. Altogether comparison with (2.2.24) yields

Proposition 2.4. The massless spectrum of type IIA strings compactified to four dimensions on a smooth internal Calabi-Yau 3-fold $X$ with full SU(3) holonomy consists of the following supermultiplets, where schematically we list the bosonic field content:

$$
\begin{array}{rlrll}
1 & \times & R(4): & \left(\mathbf{2}_{\mathbf{t}}\right) \oplus\left(\mathbf{2}_{v}\right) & \rightarrow g_{\mu \nu} \oplus C_{\mu}, \\
\left(h^{1,2}(X)+1\right) & \times & H(4): & 4\left(h^{1,2}(X)+1\right)(\mathbf{1}) & \rightarrow C_{i j k} \oplus \delta g_{i j} \oplus a \oplus \phi, \\
h^{1,1}(X) & \times & V(4): & h^{1,1}(X)\left(\mathbf{2}_{v}\right) \oplus 2 h^{1,1}(X)(\mathbf{1}) & \rightarrow C_{\mu i j} \oplus \delta g_{i \bar{\jmath}} \oplus B_{i j} .
\end{array}
$$

Heterotic $E_{8} \times E_{8}$ strings on $T^{2} \times K 3$

We now calculate the massless spectrum of heterotic $G_{10}=E_{8} \times E_{8}$ string theories compactified to four dimensions on an internal $X^{6}$ which is the product of a real two-torus $T^{2}$ and a $K 3$ surface, essentially following [Asp97]. It is easiest to use the results of Proposition 2.2 for heterotic $E_{8} \times E_{8}$ strings compactified to six dimensions on an internal $K 3$ surface, and to compactify two further space-like dimensions of $M^{1,5}$ to a two-torus $T^{2}$. As before let $K$ denote the holonomy of the gauge bundle viewed as bundle on $K 3 . K$ is the maximal simple subgroup of the centralizer for the unbroken gauge group $H$ in $E_{8} \times E_{8}$ under compactification to six dimensions with internal $K 3$. We can choose the torus $T^{2}$ together with a flat bundle with holonomy $U(1)^{r}$, and then the observed gauge group in the $D=4$ uncompactified dimensions is the centralizer of $U(1)^{r} \times K$ in $E_{8} \times E_{8}$. This implies $U(1)^{r} \subset H$, and in fact generically the observed gauge group is broken to the Abelian group $H=U(1)^{r}$.

As for type IIA strings compactified to four dimensions we can restrict to the discussion of bosonic fields, because the supermultiplets (2.2.24) are already distinguished by their bosonic field content. We decompose the relevant representations of $\mathfrak{s o}(4)$ with respect to $\mathfrak{s o}(2) \oplus \mathfrak{s o}(2)$, keeping track of the space-time indices:

$$
\mathbf{9} \longrightarrow\left(\mathbf{2}_{t}\right) \oplus 2\left(\mathbf{2}_{v}\right) \oplus 3(\mathbf{1}), \quad \mathbf{1} \longrightarrow(\mathbf{1}), \quad \mathbf{3}_{ \pm} \longrightarrow 1\left(\mathbf{2}_{v}\right) \oplus 1(\mathbf{1}), \quad \mathbf{4} \longrightarrow 1\left(\mathbf{2}_{v}\right) \oplus 2(\mathbf{1}) .
$$

Using this, let us discuss the fate of each six-dimensional supermultiplet in (2.2.4): The bosonic fields $g_{\mu \nu}, B_{\mu \nu}^{+}$of the six-dimensional supergravity multiplet contribute a four-dimensional graviton $g_{\mu \nu}$ in the $\left(\mathbf{2}_{t}\right)$, three vectors $g_{\mu a}, B_{\mu a}^{+}$in the $\left(\mathbf{2}_{v}\right)$, and four scalars $g_{a b}, B_{a b}^{+}$in the $(\mathbf{1})$, where $a, b$ denote the coordinates on $T^{2}$. The graviton and one of the vectors fill up the bosonic field content of the four-dimensional supergravity multiplet $R(4)$. The remaining two vectors and four scalars give two four-dimensional vectormultiplets $V(4)$. The bosonic fields $B_{\mu \nu}^{-}, \phi$ of each six-dimensional tensormultiplet descend to one vector $B_{\mu a}^{-}$in the $\left(\mathbf{2}_{v}\right)$ and two scalars $a=B_{\mu \nu}, \phi$ in the (1), yielding the bosonic field content of a four-dimensional vector multiplet 
$V(4)$. The only bosonic fields in a six-dimensional hypermultiplet are quadruplets of scalars, which descend to quadruplets of scalars in four dimensions, yielding the bosonic field content of a four-dimensional hypermultiplet $H(4)$. Finally, the only bosonic field in a six-dimensional vectormultiplet is the gauge field $A_{\mu}$, yielding a vector in the $\left(\mathbf{2}_{v}\right)$ and two scalars in the $(\mathbf{1})$ in four dimensions, i.e. the bosonic field content of a four-dimensional vectormultiplet $V(4)$. Summarizing, we have

$$
R(6) \longrightarrow R(4) \oplus 2 V(4), \quad T(6) \longrightarrow V(4), \quad H(6) \longrightarrow H(4), \quad V(6) \longrightarrow V(4) .
$$

Now from Proposition 2.2 we obtain

Proposition 2.5. Consider a theory which is obtained by compactification to four dimensions on an internal product of a two-torus $T^{2}$ and a smooth $K 3$ surface from a ten-dimensional $E_{8} \times E_{8}$ heterotic string theory where the gauge group on compactification to K3 is H. Moreover, assume that the bundle on the $K 3$ surface has degenerated to receive $l$ distinct pointlike instantons. Then the massless spectrum is given by

$$
R(4) \oplus(244+\operatorname{dim} H-29 l) H(4) \oplus(3+l+\operatorname{rk} H) V(4) \oplus(\operatorname{dim} H-\operatorname{rk} H) V(4) .
$$

Here, 20 hypermultiplets account for the moduli of the K3 surface, and with notations as in Proposition 2.2 at least

$$
n_{H}^{0}-20=k^{1} c_{2}\left(K^{1}\right)+k^{2} c_{2}\left(K^{2}\right)-\operatorname{dim} K+l
$$

hypermultiplets give the moduli of the gauge bundle on $K 3$, with additional contributions in special cases as remarked after Proposition 2.2. One of the vectormultiplets contains the axiondilaton pair, two of the vectormultiplets give moduli of $T^{2}$, and $\mathrm{rk} H$ vectormultiplets give moduli of the heterotic bundle on $T^{2}$. Further $l$ vectormultiplets correspond to $l$ distinct pointlike instantons. If $H$ is non-Abelian, i.e. $\operatorname{dim} H-\mathrm{rk} H \neq 0$, then one has enhanced gauge symmetry, and the additional $\operatorname{dim} H-\mathrm{rk} H$ vectormultiplets do not allow a perturbative interpretation in this heterotic theory.

\section{Anomalies}

To yield string theory as a promising approach towards describing nature, parity violation has to be incorporated in a consistent manner. However, parity violating superstring theories can suffer from anomalies. Let us give a brief summary, following [GSW87, II §10]. Helpful introductions to this topic can also be found in [AGG85, PS95, Wei05, Pol98, BM03, SS04, Adl, Har05].

Very roughly speaking, an anomaly arises when under quantization of a classical system in the resulting quantum field theory a classical symmetry is broken. In terms of the Feynman calculus there then are divergent radiative corrections which do not allow regularization. Such divergent Feynman diagrams are always one-loop diagrams [Adl69, Bar69], with a chiral fermion around the loop and a classically conserved current attached, the conservation of which is not compatible with regularization. For our purposes we need to work in $D=2 n$ space-time dimensions, and below we will see that anomalies of the type of interest here can occur only for 
odd $n$. We restrict the discussion to specific local anomalies which lead to fatal inconsistencies of the resulting quantum field theory, in contrast to global anomalies which can yield a welcome technique to break global symmetries for phenomenological reasons.

Mathematically, in order to calculate the relevant propagators in a field theory one in particular needs the determinants of those differential operators that give the equations of motion for the various fields. In the bosonic case the differential operator in question is a Laplacian, and zeta function regularization is a well-understood technique to define a determinant of it. For the fermions, however, one has to deal with families of Dirac operators, which depend on the metric of the underlying manifold and on a gauge connection. By the results of [Ati84, AS84] the existence of, say, gauge covariant propagators is obstructed by the first Chern class of the determinant of the index bundle associated to the relevant family of Dirac operators. In other words, if this bundle is non-trivial, then a gauge covariant propagator cannot be defined and the theory is anomalous. The family index theorem [AS71] hence allows to calculate all quantities that govern potential anomalies. As is explained in [ASZ84], the relevant first Chern class can be evaluated by restricting to families parametrized on two-dimensional spheres. Hence the anomaly of a theory on a $D$-dimensional spin manifold $M$ is given by the $(D+2)$ form

$$
\widehat{I}_{D+2}=(\widehat{A}(Z) \operatorname{ch}(V))_{D+2} .
$$

Here, $Z$ is a fiber bundle with base $\mathbb{S}^{2}$ and fiber $M$, i.e. it is a $(D+2)$ dimensional manifold. Hence $\widehat{A}(Z) \operatorname{ch}(V)$ is the density which occurs in the family index theorem as the curvature of the determinant bundle of the index bundle associated to a two-dimensional family of Dirac operators on $M$, coupled to a vector bundle $V$ on $M$. Indeed, $\int_{M} \widehat{A}(Z) \operatorname{ch}(V)$ gives the Chern character of the index bundle on the base $\mathbb{S}^{2}$ of $Z$, which up to a constant agrees with the first Chern class of this bundle.

As can be seen from our discussion of massless spectra in Section 2, all chiral fields in our string theories that can contribute to the bundle $V$ are sections of either a spin bundle, a RaritaSchwinger bundle, or a bundle of two-forms. For all of them the Chern classes can be expressed as combinations of the Pontrjagin classes of $M$, and therefore they can only integrate over $M$ to a non-vanishing class in degree 2 if $D=4 k+2, k \in \mathbb{N}$, which we will assume from now on. $\widehat{I}_{D+2}$ is related to the actual anomaly $G$ by a process known as transgression: $\widehat{I}_{D+2}$ is closed and gauge invariant, $d \widehat{I}_{D+2}=0=\delta \widehat{I}_{D+2}$. Hence one locally has $\widehat{I}_{D+2}=d I_{D+1}$, where $I_{D+1}$ can be viewed as closed one-form on $\mathcal{G}$ if the base $\mathbb{S}^{2}$ of $Z$ is a two-sphere in $\mathcal{M} / \mathcal{G}$. Here $\mathcal{M}$ is the infinite dimensional space of parameters (be it the space of metrics, the space of gauge connections, or the product of these two), and $\mathcal{G}$ is the infinite Lie group of symmetries of $\mathcal{M}$. In other words, $I_{D+1}$ is obtained precisely by Chern's transgression operation. Moreover, since $d \delta I_{D+1}=0$ but in general $I_{D+1}$ is not gauge invariant one finds $\delta I_{D+1}=d I_{D}$. Now $I_{D}$ can be viewed as $D$-form on $M$. It is ambiguous up to a closed form which is irrelevant for the actual anomaly $G=\int_{M} I_{D}$. The value of $G$ gives the change of the effective action under gauge or coordinate transformations, indeed the "anomaly" of the effective theory in the sense of the breaking of classical symmetries.

In [ASZ84] one also finds the following argument: While the structure group of the spin frame bundle of $Z$ in our formula for $\widehat{I}_{D+2}$ is $\mathfrak{s o}(D+2)$, it may be reduced to $\mathfrak{s o}(D) \oplus \mathfrak{s o}(2)$, and $\widehat{A}(Z)$ factors into $\mathfrak{s o}(D)$ and $\mathfrak{s o}(2)$ pieces, respectively. Only the $\mathfrak{s o}(D)$ contributions $I_{D+2}$ 
are relevant, since on the one hand the $\mathfrak{s o}(2)$ pieces are universal, and on the other hand approximating them by 1 is sufficient for checking consistency by results of [WZ71, BZ84]. Note that this approximation, strictly speaking, does not restrict the relevant contributions to $\widehat{I}_{D+2}$ to forms on $M$. However, in all calculations this subtlety is irrelevant: $\widehat{I}_{D+2}$ is viewed as a formal object, given that $(D+2)$ forms on $M$ do not make sense, but solely the dependence on the coordinates of $M$ is of interest. To implement this, let $\mathcal{R}$ be the Riemann curvature of the spin frame bundle of $Z$ and $\bar{R}$ the Riemann curvature of $M$. According to the splitting principle, there exist two-forms $x_{0}, x_{1}, \ldots, x_{2 k+1}$ such that

$$
\frac{1}{2}\left(-\frac{1}{4}\right)^{m} \operatorname{tr}\left(\mathcal{R}^{2 m}\right)=\frac{1}{4^{m}} \sum_{i=0}^{2 k+1} x_{i}^{2 m} \quad \longrightarrow \quad Y_{2 m}:=\frac{1}{4^{m}} \sum_{i=1}^{2 k+1} x_{i}^{2 m}=\frac{1}{2}\left(-\frac{1}{4}\right)^{m} \operatorname{tr}\left(\bar{R}^{2 m}\right),
$$

where in restricting attention to $Y_{2 m}$ we assume that $x_{0}$ is the $\mathfrak{s o}(2)$ piece of the curvature, while the $x_{i}$ with $i>0$ give the $\mathfrak{s o}(D)$ pieces which are relevant for our discussion. We also follow [GSW87] and [Sch02] in surpressing the dependence on the base of $Z$.

\subsection{Gravitational and gauge anomalies}

By the above, potential anomalies of the type we are interested in can only occur for string theories with chiral fields in an external space-time of dimension $D=4 k+2, k \in \mathbb{N}$. Of the theories considered in Section 2 this amounts to the cases $D=10$ and $D=6$. Contributions to the anomaly can come from space-time spinors $\Psi^{\mp}, \Lambda^{+}, \psi^{-}, \chi^{-}, \lambda^{+}$, i.e. from spin $\frac{1}{2}$ fields, or from Rarita-Schwinger fields $\Psi_{M}^{ \pm}, \psi_{\mu}^{ \pm}$, i.e. from spin $\frac{3}{2}$ fields, or from chiral two-forms $B_{\mu \nu}^{ \pm}$. The indices of the respective Dirac operators contribute with a sign according to their chirality. This implies that theories of type IIA can never suffer from anomalies of this type, since spinors occur only in pairs of opposite chiralities in these theories, with their contributions to the anomaly cancelling.

Among the theories discussed in Section 2 we therefore only need to consider the heterotic $E_{8} \times E_{8}$ theory in $D=10$ space-time dimensions, see (2.1.3), and the one in $D=6$ space-time dimensions with internal $K 3$ surface, see Proposition 2.2. The heterotic $E_{8} \times E_{8}$ theories with internal real four-torus are anomaly free, since these theories enjoy enhanced supersymmetry, and their spectrum is non-chiral, as can be seen from Proposition 2.3.

Before discussing anomaly cancellation, let us determine the form of each possible contribution to the anomaly. This is very nicely explained in [ASZ84]. As a warm-up, consider a chiral spin $\frac{1}{2}$ field, i.e. a section $\psi^{+}$of the spinor bundle $S^{+}$which obeys $\not D \psi^{+}=0$ for the ordinary untwisted Dirac operator $\not D: S^{+} \longrightarrow S^{-}$. We need the index bundle of the family $\not D$ over $\mathcal{M}$, which depends on the curvature $\bar{R}$,

$$
I_{1 / 2}(\bar{R})=\widehat{A}(\bar{R})_{4 k+4} \quad \text { where } \quad \widehat{A}(\bar{R})=\prod_{i=1}^{2 k+1} \frac{\frac{1}{2} x_{i}}{\sinh \frac{1}{2} x_{i}} .
$$

More generally a spinor with values in some gauge bundle of curvature $\bar{F}$ contributes an anomaly given by the index of the corresponding twisted Dirac operator,

$$
I_{1 / 2}(\bar{R}, F)=\left(\left(\operatorname{tr}\left(\mathrm{e}^{\mathrm{iF}}\right) \widehat{\mathrm{A}}(\overline{\mathrm{R}})\right)\right)_{4 k+4}, \quad \text { where } \quad I_{1 / 2}(\bar{R})=I_{1 / 2}(\bar{R}, 0)
$$


For Rarita-Schwinger fields one needs the index of the Rarita-Schwinger complex (A.6). The contributions $\ominus S^{+} \ominus S^{-}$which equally occur in the domain and in the image of the RaritaSchwinger operator $\not_{R S}$ cancel out, leaving us with the Dirac operator on $\left(S^{+} \otimes T^{*} M\right) \ominus S^{+}=$ $S^{+} \otimes\left(T^{*} M \ominus \mathbf{1}\right)$, see [ASZ84, $\left.\S \mathrm{IV} . \mathrm{V}\right]$. In the physics literature, the virtual subtraction of $\mathbf{1}$ is referred to as subtracting ghost contributions. The relevant index is hence given by

$$
I_{3 / 2}(\bar{R})=\left(\widehat{A}(\bar{R})\left(2 \sum_{i=1}^{2 k+1} \cosh x_{i}-1\right)\right)_{4 k+4} .
$$

Finally, for chiral two-forms one needs the density of the twisted Dirac operator $\not D_{A}: S^{+} \otimes$ $S^{-} \longrightarrow S^{-} \otimes S^{-}$, i.e.

$$
I_{A}(\bar{R})=-\frac{1}{8} L(\bar{R})_{4 k+4}, \quad \text { where } \quad L(\bar{R})=\prod_{i=1}^{2 k+1} \frac{x_{i}}{\tanh x_{i}}
$$

the Hirzebruch $L$-genus.

Altogether every contribution to the anomaly can be expressed in terms of known universal polynomials in the $Y_{2 m}$. For later convenience let us give the result for the various anomalies: For chiral spinors governed by a twisted Dirac operator let $g:=\operatorname{tr}(1)$, the dimension of the representation to which our chiral spinor belongs, and $g=1$ for $F=0$. For $D=10$ one finds [GSW85, II, pp. 351-352],

$$
\begin{aligned}
I_{3 / 2}(\bar{R})= & \frac{1}{45360}\left(7920 Y_{6}-9450 Y_{2} Y_{4}+2205 Y_{2}^{3}\right), \\
I_{A}(\bar{R})= & \frac{1}{45360}\left(-7936 Y_{6}+9408 Y_{2} Y_{4}-2240 Y_{2}^{3}\right), \\
I_{1 / 2}(\bar{R}, \bar{F})= & -\frac{1}{720} \operatorname{tr} F^{6}-\frac{1}{144} \operatorname{tr} F^{4} Y_{2}-\frac{1}{8} \operatorname{tr} F^{2}\left(\frac{1}{45} Y_{4}+\frac{1}{18} Y_{2}^{2}\right) \\
& -g\left(\frac{1}{2835} Y_{6}+\frac{1}{1080} Y_{2} Y_{4}+\frac{1}{1296} Y_{2}^{3}\right),
\end{aligned}
$$

while for $D=6$ we have [GSW87, II, pp. 349-351]

$$
\begin{aligned}
I_{3 / 2}(\bar{R}) & =\frac{1}{72}\left(-43 Y_{2}^{2}+98 Y_{4}\right) \\
I_{A}(\bar{R}) & =-\frac{1}{45}\left(5 Y_{2}^{2}-7 Y_{4}\right) \\
I_{1 / 2}(\bar{R}, \bar{F}) & =\frac{1}{24} \operatorname{tr} F^{4}+\frac{1}{12} \operatorname{tr} F^{2} Y_{2}+g\left(\frac{1}{180} Y_{4}+\frac{1}{72} Y_{2}^{2}\right) .
\end{aligned}
$$

\subsection{Anomaly cancellation}

Before turning to the discussion of anomaly cancellation for the heterotic $E_{8} \times E_{8}$ theories, let us mention that for $D=10$ from (3.1.1) one obtains

$$
I_{3 / 2}(\bar{R})+I_{A}(\bar{R})-I_{1 / 2}(\bar{R}, 0)=0 .
$$


This means that a ten-dimensional theory is free of anomalies if it has as its chiral field content $m$ complex negative chirality spin $\frac{1}{2}$ field, $m$ complex positive chirality spin $\frac{3}{2}$ field, and $m$ real self-dual antisymmetric tensor. In fact, with $m=2$ this is precisely the chiral field content of type IIB string theory. This surprising fact was first observed in [AGW84].

\subsubsection{Anomaly free heterotic $E_{8} \times E_{8}$ theories in $D=10$ dimensions}

Let us now turn to heterotic $G_{10}=E_{8} \times E_{8}$ theories in ten dimensions. According to (2.1.3) we obtain contributions to the anomaly from one gravitino $\Psi_{M}^{+}$, one dilatino $\Psi^{-}$, and one gaugino $\Lambda^{+}$, such that the anomaly is

$$
\widehat{I}_{12}=I_{3 / 2}(\bar{R})-I_{1 / 2}(\bar{R}, 0)+I_{1 / 2}(\bar{R}, \bar{F}) .
$$

Using (3.1.1) one determines the coefficient of $Y_{6}$ in $\widehat{I}_{12}$ to be a multiple of $(496-g)$, which vanishes precisely because $g=\operatorname{dim} G_{10}=496$.

Although this is an encouraging start, in pure supergravity in $D=4 k+2$ dimensions the total anomaly $\widehat{I}_{4 k+4}$ turns out to never vanish. However, according to the seminal papers [GS84, GS85b, GS85a], in heterotic theories a further anomalous diagram occurs which we have not yet discussed. It is a tree diagram in which the massless 2-form $B$ of the supergravity multiplet is exchanged between two gauge bosons and either two gluons, or two gravitinos, or four gravitons. In more mathematical terms, as already mentioned in the footnote on page 5 , the B-field is only locally given by a closed 2-form. It is a closed differential cochain and in particular transforms non-trivially under gauge transformations. Accordingly, it does contribute to gauge anomalies. In fact, the non-trivial Yang-Mills gauge transformation of the $B$-field gives a potential for a gauge-invariant three-form field strength $H$. With $\omega_{L}$ and $\omega_{Y}$ denoting the Lorentz and Yang-Mills Chern-Simons forms, the latter obeys

$$
H=d B+\omega_{L}+\alpha \omega_{Y} \quad \Longrightarrow \quad d H=\operatorname{tr}\left(\overline{\mathrm{R}}^{2}\right)+\alpha \operatorname{Tr}\left(\overline{\mathrm{F}}^{2}\right) .
$$

The anomalous tree diagram therefore contributes a term $\left(\alpha \operatorname{Tr}\left(\overline{\mathrm{F}}^{2}\right)+\operatorname{tr}\left(\overline{\mathrm{R}}^{2}\right)\right) \mathrm{X}_{4 \mathrm{k}}$ with a $4 k$-form $X_{4 k}$. Hence to cancel the anomaly $\widehat{I}_{4 k+4}$, we must have

$$
\widehat{I}_{4 k+4} \sim\left(\alpha \operatorname{Tr}\left(\overline{\mathrm{F}}^{2}\right)+\operatorname{tr}\left(\overline{\mathrm{R}}^{2}\right)\right) \mathrm{X}_{4 \mathrm{k}} .
$$

One can show (see e.g. [GSW85]) that in ten dimensions this factorization holds if $G_{10}=E_{8} \times E_{8}$ or $G_{10}=\mathrm{SO}(32) / \mathbb{Z}_{2}$ and with $\alpha=-1 / 30$ (note that also $\operatorname{dim} \mathrm{SO}(32) / \mathbb{Z}_{2}=496$ ), so that anomalies are indeed cancelled in these theories. If $X_{4 k}$ can be integrated to $d X_{4 k-1}=X_{4 k}$, then the factorization (3.2.2) together with the known properties of the Chern-Simons forms allow to solve the descent equations and to calculate the actual anomaly coming from the tree diagram for the exchange of $B$ described above.

The process of anomaly cancellation described here is known as the Green-Schwarz mechanism [GS84, GS85b, GS85a]. It remains one of the mysteries of string theory to understand why it works, and its discovery meant a break-through that triggered what is now known as the First String Revolution. 


\subsubsection{Anomaly free heterotic $E_{8} \times E_{8}$ theories in $D=6$ dimensions}

Let us determine when a heterotic $E_{8} \times E_{8}$ string theory in $D=6$ dimensions with an internal $K 3$ surface is anomaly free. We know that its massless spectrum is of the form (2.2.5). The requirement that the theory be anomaly-free poses a restriction on the numbers $n_{T}, n_{V}, n_{H}$. The Green-Schwarz mechanism described above applies just as in the ten-dimensional situation. It implies, by integration of (3.2.1) over the internal $K 3$ surface, that the instanton number (2.2.12) of the heterotic bundle viewed as a bundle on $K 3$ is 24 (see (2.2.22)). The GreenSchwarz mechanism cancels all contributions to the anomaly by terms of the form (3.2.2), apart from those proportional to $Y_{4} \sim \operatorname{tr}\left(\bar{R}^{4}\right)$. Hence in order to check anomaly cancellation we need to collect the contributions to the coefficient of $Y_{4}$ in the total anomaly, carefully taking into account the various fields in our multiplets and their chiralities.

The fields from the supergravity multiplet $R(6)$ of (2.2.4) which contribute to the anomaly are the gravitino $\psi_{\mu}^{+}$and the self-dual tensor field $B_{\mu \nu}^{+}$, both of positive chirality. From (3.1.2) we read the coefficient in front of $Y_{4}$,

$$
\frac{98}{72}+\frac{7}{45}=\frac{1}{180}(245+28)=\frac{273}{180}
$$

The fields from a tensormultiplet $T(6)$ of (2.2.4) which contribute to the anomaly are the antiself-dual tensor field $B_{\mu \nu}^{-}$and the spinor $\psi^{-}$, both of negative chirality, yielding the following coefficient in front of $Y_{4}$,

$$
-\frac{7}{45}-\frac{1}{180}=-\frac{1}{180}(28+1)=-\frac{29}{180}
$$

The only field from each vectormultiplet $V(6)$ of (2.2.4) contributing to the anomaly is the spinor $\lambda^{+}$of positive chirality, giving a coefficient

$$
\frac{1}{180}
$$

while from each hypermultiplet we get a contribution from the spinor $\chi^{-}$of negative chirality, thus the coefficient

$$
-\frac{1}{180}
$$

in front of $Y_{4}$. Adding everything up we find

Proposition 3.1. A heterotic $E_{8} \times E_{8}$ string theory in $D=6$ dimensions with internal $K 3$ surface and $n_{T}, n_{V}, n_{H}$ tensor-, vector-and hypermultiplets, respectively, is anomaly free iff

$$
n_{H}-n_{V}=273-29 n_{T}
$$

and the instanton number is 24 .

In Proposition 2.2 we have determined the massless spectrum for examples of theories of the type addressed in the above proposition. Namely, consider a theory which is obtained by compactification from a ten-dimensional $E_{8} \times E_{8}$ heterotic theory to six dimensions on a smooth 
internal $K 3$ surface. Denote the unbroken gauge group by $H$ and assume furthermore that the bundle on $K 3$ has degenerated to receive $l$ distinct pointlike instantons. Then according to Proposition 2.2 the massless spectrum obeys

$$
n_{T}=l+1, \quad n_{V}=\operatorname{dim}(H), \quad n_{H}=244+\operatorname{dim}(H)-29 l .
$$

We immediately see that the anomaly cancellation condition of Proposition 3.1 holds. Note that we have thus given two independent derivations for the formula (3.2.3) for this case. In other words, heterotic string theories arising from compactification of anomaly free theories in ten dimensions are automatically anomaly free, and this remains true when the gauge bundle acquires pointlike instantons.

\subsection{Examples}

This section is devoted to the presentation of a number of examples, where we carry out the calculations of the numbers $n_{T}, n_{V}, n_{H}^{0}$, and $n_{H}^{c h}$ explained above. In view of the heterotic F-theory duality these examples are representative, as we shall see in Section 5.3. In each case, anomaly cancellation (3.2.3) holds.

Assume that the heterotic bundle data specify two $E_{8}$ bundles with instanton numbers $k^{1}, k^{2}$ and $l^{1}, l^{2}$ distinct pointlike instantons, respectively, where the holonomy is given by $K^{i} \subset E_{8}$ and by (2.2.22) we have $k^{1}+k^{2}+l^{1}+l^{2}=24$. In this case the unbroken gauge group of the heterotic theory is $H^{1} \times H^{2}$ with $H^{i} \subset E_{8}$ the centralizer of $K^{i}$ in $E_{8}$. We use the unbroken gauge group $H^{1} \times H^{2}$ to label these examples as in [BIK $\left.{ }^{+} 96\right]$, where a similar analysis is performed.

\subsubsection{Completely broken gauge group}

If the gauge group is completely broken, the $K 3$ bundle has holonomy $K=E_{8} \times E_{8}$. Assuming no pointlike instantons, by Proposition 2.2 and (2.2.23)

$$
n_{V}=0, \quad n_{T}=1, \quad n_{H}^{0}=20+30\left(k^{1}+k^{2}\right)-496=244, \quad n_{H}^{c h}=0 .
$$

\subsubsection{Unbroken $E_{8}$ gauge group}

With gauge group $H=E_{8} \times\{\operatorname{id}\}$ we have holonomy $K=\{\operatorname{id}\} \times E_{8}$. An $E_{8}$ bundle can have trivial holonomy only if all its curvature is concentrated in pointlike instantons. So let us assume that there are $l=l^{1}$ distinct pointlike instantons, $k^{1}=0$, while the second bundle has full $E_{8}$ holonomy with instanton number $k^{2}$ and we assume $l^{2}=0$. From (2.2.7) we see that there is no charged matter, such that with Proposition 2.2 and (2.2.23)

$$
n_{V}=248, \quad n_{T}=l+1, \quad n_{H}^{0}=20+30 k^{2}-248+l=492-29 l, \quad n_{H}^{c h}=0 .
$$

\subsubsection{Unbroken $E_{7}$ gauge group}

With gauge group $H=E_{7} \times\{\mathrm{id}\}$ we have holonomy $K=\mathrm{SU}(2) \times E_{8}$. Assume that there are $l$ distinct pointlike instantons on the bundle with $\mathrm{SU}(2)$ holonomy and effective instanton number 
$k^{1}$, while the second bundle has full $E_{8}$ holonomy with instanton number $k^{2}$. From (2.2.7) we see that there is a contribution to the charged matter, $248=(\mathbf{1 3 3}, \mathbf{1}) \oplus(\mathbf{5 6}, \mathbf{2}) \oplus(\mathbf{1}, \mathbf{3})$, in which $(\mathbf{5 6}, \mathbf{2})$ gives charged hypermultiplets. The multiplicity is computed from $(2.2 .18)$ and (2.2.19) and amounts to

$$
N(\mathbf{5 6})=k^{1} \text { ind }(\mathbf{2})-\operatorname{dim}(\mathbf{2})=\frac{1}{2} k^{1}-2 .
$$

In total from Proposition 2.2 and (2.2.23) we have

$$
\begin{aligned}
n_{V}=133, n_{T}=l+1, & n_{H}^{0}=20+2 k^{1}+30 k^{2}-251+l=-231+2 k^{1}+30 k^{2}+l, \\
n_{H}^{c h} & =28 k^{1}-112 .
\end{aligned}
$$

\subsubsection{Unbroken $E_{6}$ gauge group}

With gauge group $H=E_{6} \times\{\mathrm{id}\}$ we have holonomy $K=\mathrm{SU}(3) \times E_{8}$. Assume that there are $l$ distinct pointlike instantons on the bundle with $\mathrm{SU}(3)$ holonomy and effective instanton number $k^{1}$, while the second bundle has full $E_{8}$ holonomy with instanton number $k^{2}$. From (2.2.7) we see that there is a contribution to the charged matter, $\mathbf{2 4 8}=(\mathbf{7 8}, \mathbf{1}) \oplus(\mathbf{2 7}, \mathbf{3}) \oplus(\overline{\mathbf{2 7}}, \overline{\mathbf{3}}) \oplus(\mathbf{1}, \mathbf{8})$, in which $(\mathbf{2 7}, \mathbf{3}) \oplus(\overline{\mathbf{2 7}}, \overline{\mathbf{3}})$ gives charged hypermultiplets. The multiplicity is computed from (2.2.18) and (2.2.19) and amounts to

$$
N(\mathbf{2 7})=N(\overline{\mathbf{2 7}})=k^{1} \text { ind }(\mathbf{3})-\operatorname{dim}(\mathbf{3})=\frac{1}{2} k^{1}-3 .
$$

To ease computations like this one, the book [MP81] is recommended, where indices of representations like the ones that occur here are tabulated. In total from Proposition 2.2 and (2.2.23) we have

$$
\begin{aligned}
n_{V}=78, \quad n_{T}=l+1, & n_{H}^{0}=20+3 k^{1}+30 k^{2}-256+l=-236+3 k^{1}+30 k^{2}+l, \\
& n_{H}^{c h}=27 k^{1}-162 .
\end{aligned}
$$

\subsubsection{Unbroken $F_{4}$ gauge group}

With gauge group $H=F_{4} \times\{$ id $\}$ we have holonomy $K=G_{2} \times E_{8}$. From (2.2.7) we see that there is a contribution to the charged matter, $248=(\mathbf{5 2}, \mathbf{1}) \oplus(\mathbf{2 6}, \mathbf{7}) \oplus(\mathbf{1}, \mathbf{1 4})$, in which $(\mathbf{2 6}, \mathbf{7})$ contributes charged hypermultiplets. However, in this case a two-dimensional subspace of the $\mathbf{2 6}$ is in fact uncharged under the gauge group, i.e. the kernel of the representation when restricted to the Cartan torus $\mathfrak{t}$ of $H$ is two-dimensional. The corresponding multiplets contribute to $n_{H}^{0}$ rather than $n_{H}^{c h}$ (see the remark after Proposition 2.2). The multiplicity of 26 obtained from (2.2.18) and (2.2.19) is

$$
N(\mathbf{2 6})=k^{1} \text { ind }(\mathbf{7})-\operatorname{dim}(\mathbf{7})=k^{1}-7 .
$$

In total from Proposition 2.2 and (2.2.23) we have

$$
\begin{aligned}
n_{V}=52, \quad n_{T}=1, & n_{H}^{0}=20+4 k^{1}+30 k^{2}-262+2\left(k^{1}-7\right)=-256+6 k^{1}+30 k^{2} \\
n_{H}^{c h} & =24 k^{1}-168 .
\end{aligned}
$$




\subsubsection{Unbroken $\operatorname{Spin}(10)$ gauge group}

With gauge group $H=\operatorname{Spin}(10) \times\{$ id $\}$ we have holonomy $K=\mathrm{SU}(4) \times E_{8}$. From $(2.2 .7)$ we see that there is a contribution to the charged matter, $248=(\mathbf{4 5}, \mathbf{1}) \oplus(\mathbf{1 0}, \mathbf{6}) \oplus(\mathbf{1 6}, \mathbf{4} \oplus \mathbf{4}) \oplus(\mathbf{1}, \mathbf{1 5})$, in which $(\mathbf{1 0}, \mathbf{6}) \oplus(\mathbf{1 6}, \mathbf{4} \oplus \mathbf{4})$ contributes charged hypermultiplets. The multiplicities obtained from (2.2.18) and (2.2.19) are

$$
N(\mathbf{1 0})=k^{1} \text { ind }(\mathbf{6})-\operatorname{dim}(\mathbf{6})=k^{1}-6, \quad N(\mathbf{1 6})=2\left(k^{1} \text { ind }(\mathbf{4})-\operatorname{dim}(\mathbf{4})\right)=k^{1}-8 .
$$

In total from Proposition 2.2 and (2.2.23) we have

$$
\begin{aligned}
n_{V}=45, \quad n_{T}=1, & n_{H}^{0}=20+4 k^{1}+30 k^{2}-263=-243+4 k^{1}+30 k^{2}, \\
n_{H}^{c h} & =10\left(k^{1}-6\right)+16\left(k^{1}-8\right)=26 k^{1}-188 .
\end{aligned}
$$

\section{Heterotic - type IIA and F-theory duality}

While the five basic string theories in ten-dimensional Minkowski space can be distinguished by their numbers of supersymmetries and their massless field content, after compactification to $D$ dimensions with $D<10$ this is not true anymore. As a consequence, so-called string-string dualities between various string theories were conjectured. In fact it is claimed that all string theories are connected by a web of dualities. Here we concentrate on the heterotic - type IIA duality and the heterotic - F-theory duality. For our purposes, the latter is best viewed as a certain limit of the former.

\subsection{The heterotic - type IIA duality}

We have already encountered an example of the phenomenon known as a string-string duality. Namely, in Proposition 2.3 we observed that in compactifications to six dimensions the massless spectra agree for heterotic $E_{8} \times E_{8}$ strings with internal real four-torus on the one hand and for type IIA strings with internal $K 3$ surface on the other hand. In fact, more can be said since the (classical) moduli spaces of the respective theories are known explicitly. The scalars in the massless supermultiplets give real coordinates of these moduli spaces, and for both theories one finds the moduli space

$$
\mathbb{R} \times \mathrm{O}^{+}(4,20 ; \mathbb{Z}) \backslash \mathrm{O}^{+}(4,20 ; \mathbb{R}) /(\mathrm{SO}(4) \times \mathrm{O}(20)),
$$

where the factor $\mathbb{R}$ accounts for the dilaton, and the remaining 80-dimensional quaternionic Kähler space corresponds to the $20 \times 4$ scalars from the 20 hypermultiplets.

On the basis of these stunning agreements physicists have made the daring conjecture [HT95, Wit95] that these string theories in fact are equivalent. One says that there is a string-string duality between heterotic and type IIA theories, respectively. Much evidence in favor of this conjectured duality has been collected, including the fact that the low energy effective actions agree [Wit95]. 
It is important to note that this string-string duality cannot be seen purely perturbatively, since it maps the heterotic dilaton to the negative of the type IIA dilaton [Wit95], and thus the string coupling constant is inverted under the duality. Nevertheless, we have geometric interpretations of all scalars in the hypermultiplets in terms of an Einstein metric, a B-field, and a connection one-form of a flat bundle on a real four-torus on the one hand, and in terms of an Einstein metric and a B-field on a $K 3$ surface on the other hand. Hence the conjectured duality induces a map between these geometric structures. This can be made very explicit as follows: For the $K 3$ surface on the type IIA side we can always choose a complex structure such that this surface is elliptically fibered with section. In standard (singular) Weierstraß form it is given by an equation

$$
y^{2}=x^{3}+x f(z)+g(z), \quad \text { with } \quad f(z)=\sum_{m=0}^{8} f^{(m)} z^{m}, \quad g(z)=\sum_{n=0}^{12} g^{(n)} z^{n},
$$

where $(x, y)$ are affine coordinates in $\mathbb{C P}^{2}$ for the fiber, while $z$ is an affine coordinate in the base $\mathbb{C P}^{1}$ of the fibration and $f^{(j)}, g^{(k)}$ are complex constants. We explicitly allow degenerations of our $K 3$ surface where it obtains singularities of ADE type: For example, if $f(z)=\alpha z^{4}$ and $g(z)=z^{5}+\beta z^{6}+z^{7}$ with $\alpha, \beta \in \mathbb{C}$, then near $z=0$ (and similarly near $z=\infty$ ), the equation (4.1.2) gives

$$
y^{2}=x^{3}+z^{5}+\ldots,
$$

with the familiar exponents $(2,3,5)$ of an $E_{8}$ quotient singularity. The actual $K 3$ surface is obtained by minimally resolving all such singularities. This is why the above model is called the singular Weierstraß model. The possible singular fibers have been classified by Kodaira [Kod64].

More generally note that the Grassmannian $\mathrm{O}^{+}(4,20 ; \mathbb{R}) / \mathrm{SO}(4) \times \mathrm{O}(20)$ of $(4.1 .1)$ is modelled on the cohomology of $K 3, H^{*}(K 3, \mathbb{R}) \cong \mathbb{R}^{4,20}$, and its points are given by positive definite oriented four-planes in $H^{*}(K 3, \mathbb{R})$ which encode the geometric data of a real Einstein metric and a B-field on $K 3$ [AM94]. If the $K 3$ surfaces under inspection are restricted to have specific singularities, then this amounts to restricting these four-planes to $E^{\perp}$, where $E \subset H^{\text {even }}(K 3, \mathbb{Z})$ is the lattice associated to the exceptional divisor in the resolution of that singularity. Hence with $m=\mathrm{rk} E$ the Grassmannian factor in (4.1.1) reduces to $\mathrm{O}^{+}(4,20-m ; \mathbb{R}) / \mathrm{SO}(4) \times \mathrm{O}(20-m)$. In the interpretation of (4.1.1) as moduli space of real four-tori equipped with semi-stable $E_{8} \times E_{8}$ bundles, the restriction of four-planes to $E^{\perp}$ amounts to restricting to bundles on the four-torus with some unbroken gauge symmetry, i.e. with restricted holonomy. Specifically, to the lattice $E$ one can associated a semi-simple Lie algebra $\mathfrak{g} \subset \mathfrak{e}_{8} \oplus \mathfrak{e}_{8}$ because $E$ comes from a collection of singularities of ADE type on $K 3$. The restricted holonomy of the gauge bundle then is the centralizer $K$ of $G \subset E_{8} \times E_{8}$, where $G$ has Lie algebra $\mathfrak{g}$. In particular, in the above example the toroidal bundle has trivial holonomy and full $E_{8} \times E_{8}$ gauge symmetry with $\mathfrak{g}=\mathfrak{e}_{8} \oplus \mathfrak{e}_{8}$.

One finds [MV96b] that the coefficients $f^{(j)}, g^{(k)}$ in (4.1.2) with $j \leq 3, k \leq 5$ give the data of one of the heterotic $E_{8}$ bundles, while the data of the second heterotic $E_{8}$ bundle are encoded in the coefficients with $j \geq 5, k \geq 7$. The remaining two parameters $f^{(4)}$ and $g^{(6)}$ on the heterotic side are interpreted as specifying complex structure and complexified Kähler structure of an elliptic curve. This may be surprising, since we expect to find the geometric data of a real four-torus. However, for such a torus one can always choose a complex structure such that 
it is elliptically fibered. Then $f^{(4)}$ and $g^{(6)}$ only give the data of the base of such a fibration. Correspondingly on the type IIA side we have not specified the Kähler class of our $K 3$ surface.

Following [HT95, FHSV95, KV95, Sen96a, Vaf96, DMW96], the conjectured heterotic - type IIA duality in six dimensions has been generalized to a conjectured heterotic - type IIA duality in four dimensions. Surprisingly, the following naive idea seems to work: Consider heterotic $E_{8} \times E_{8}$ strings in six dimensions with internal space the product of a real two-torus and a $K 3$ surface. Using an elliptic fibration of the $K 3$ surface, the complex structure of the internal space can always be chosen such that it is a fibration with section over $\mathbb{C P}^{1}$ and with generic fiber a complex two-torus. A fiberwise application of the six-dimensional heterotic - type IIA duality yields this theory dual to a type IIA theory in four dimensions with internal Calabi-Yau 3 -fold which is $K 3$-fibered over the "same" $\mathbb{C P}^{1}$ we used on the heterotic side. We will see below that this idea carries tremendously far.

Note that any heterotic - type IIA duality in four dimensions requires a matching of the massless spectra found in Propositions 2.4 and 2.5, respectively. Particularly for heterotic theories on a product of a real two-torus and a $K 3$ surface, the form of the moduli space associated to the scalars which give geometric moduli in the vectormultiplets is known, at least at small string coupling, where perturbative techniques hold: With $m=$ rk $H$ the rank of the unbroken gauge group $H$ on compactification to $K 3$ as in Proposition 2.5, one has a space of the form

$$
\mathrm{O}^{+}(2,2+m ; \mathbb{Z}) \backslash \mathrm{O}^{+}(2,2+m ; \mathbb{R}) /(\mathrm{SO}(2) \times \mathrm{O}(2+m)) \quad \times \quad \mathrm{SU}(2) / U(1),
$$

where the second factor accounts for the axion-dilaton pair. If a dual type IIA theory exists, then the moduli space formed by the scalars of $(m+3)$ of its vectormultiplets must take the same form as above in a regime where some parameter corresponding to the heterotic dilaton becomes small. In [AL96] it was shown that this implies that the respective type IIA theory has an internal Calabi-Yau 3-fold which is $K 3$-fibered. If this fibration has a section, then the size of the section corresponds to the value of the heterotic dilaton.

To match the hypermultiplet spectrum recall that on the heterotic side the hypermultiplets appear in two disguises, neutral and charged (2.2.23). On the type IIA side all $\left(h^{1,2}(X)+1\right)$ hypermultiplets of Proposition 2.4 are neutral. The charged hypermultiplets (as well as $(\operatorname{dim} H-\mathrm{rk} H$ ) of the vectormultiplets in Proposition 2.5) arise from non-perturbative phenomena which we have not yet accounted for on the type IIA side, since we have always assumed the Calabi-Yau 3 -fold to be smooth. On the heterotic side this restriction amounts to assuming Abelian gauge groups $H$ where no enhanced symmetry and no charged matter occurs. Using Propositions 2.4 and 2.5 we altogether have

Conjecture 4.1. There exists a duality between four-dimensional string theories which maps a heterotic $E_{8} \times E_{8}$ string theory on a product of an elliptic curve and a K3 surface to a type IIA theory with an internal Calabi-Yau 3-fold $X$ which is K3-fibered, if there exists a regime where both the respective perturbation theories converge. Moreover, if the heterotic theory arises from compactification from a ten-dimensional theory such that the gauge group on compactification to $K 3$ is $H$ and the gauge bundle viewed as a bundle on $K 3$ acquires $l$ distinct pointlike instantons, then $X$ has Hodge numbers

$$
h^{1,2}(X)=n_{H}^{0}-1=19+k^{1} c_{2}\left(K^{1}\right)+k^{2} c_{2}\left(K^{2}\right)-\operatorname{dim} K+l, \quad h^{1,1}(X)=3+\mathrm{rk} H+l
$$


with notations as in Proposition 2.2 and with the comment concerning a possible enhancement of $n_{H}^{0}$ as stated after that proposition.

If in addition the Calabi-Yau 3-fold $X$ on the type IIA side of the duality is elliptically fibered, then to capture its complex structure data it is often convenient to use a singular Weierstraß form (4.1.2), where now the coefficients of the polynomials $f$ and $g$ depend on a second affine parameter $z_{2}$ of the base $\mathbb{C P}^{1}$ of the $K 3$-fibration of $X$ :

$$
y^{2}=x^{3}+x \sum_{m=0}^{8} z_{1}^{m} f^{(m)}\left(z_{2}\right)+\sum_{n=0}^{12} z_{1}^{n} g^{(n)}\left(z_{2}\right)
$$

with $\operatorname{deg} f^{(i)}=8$ and $\operatorname{deg} g^{(j)}=12$. Generalizing the discussion in the case of an elliptically fibered $K 3$ surface and according to physics lore [MV96b, BIK ${ }^{+} 96$ ] the various parameters in (4.1.4) are assigned an interpretation in the dual heterotic theory. Namely, the parameters governing the polynomials $f^{(0)}, \ldots, f^{(3)}, g^{(0)}, \ldots, g^{(5)}$ correspond to the bundle parameters of one $E_{8}$ bundle, those governing the polynomials $f^{(5)}, \ldots, f^{(8)}, g^{(7)}, \ldots, g^{(12)}$ correspond to the bundle parameters of the second $E_{8}$ bundle, while $f^{(4)}$ and $g^{(6)}$ give the complex structure data of the $K 3$ surface in the heterotic product of an elliptic curve with $K 3$. This description can be very useful, for instance because in many examples a parameter count in the polynomials already leads to a correct determination of $h^{1,2}(X)$. Moreover, this setting is tailor made for the application of a spectral cover description of the heterotic bundles [FMW97, BJPS97, BCG ${ }^{+}$98]. However, the ansatz has to be handled with care. First, the parameter count only works when all complex structure deformations of $X$ respect the algebraic form of (4.1.4). It is not hard to construct examples where not all contributions to $h^{1,2}(X)$ are visible in terms of a parameter count in (4.1.4). Second, the assumption that a global form (4.1.4) of the equation for $X$ exists does not always hold, because globally one need not have coordinate transforms that yield all fibers of an elliptic fibration in Weierstraß form. Finally, in (4.1.4) one has tacitly assumed that all fibrations have at least one global section.

\subsection{The F-theory limit}

For the heterotic - type IIA duality in four dimensions our presentation of the matching of multiplets in Conjecture 4.1 so far is exact only for Abelian gauge groups $H$. This is the generic case, but in our Proposition 2.5 we have already accounted for the possibility of enhanced gauge symmetry on the heterotic side, which goes along with the appearance of charged hypermultiplets. The natural setup for considering non-Abelian gauge groups is a decompactification limit of the real two-torus in the heterotic theory. To perform such an operation, in (4.1.3) one has to choose a subspace of the form $\mathrm{O}^{+}(2,2 ; \mathbb{Z}) \backslash \mathrm{O}^{+}(2,2 ; \mathbb{R}) / \mathrm{SO}(2) \times O(2)$, singling out the parameters of $T^{2}$ to make its volume large. In this limit, all parameters of $T^{2}$ are lost, and the theory becomes effectively six-dimensional. In particular, the gauge group is the centralizer in $E_{8} \times E_{8}$ of the holonomy group of the $K 3$-bundle. In general this can be a non-Abelian group, and the charged hypermultiplets capture the respective decomposition of the holonomy representation as in (2.2.7).

On the type IIA side, this decompactification process corresponds to taking a so-called Ftheory limit. The physics literature on this theme is vast, see for example [Vaf96, Sen96b, 
Wit95, MV96a, MV96b, BIK ${ }^{+} 96$, AM97]. The singling out of heterotic $T^{2}$ parameters in terms of $\mathrm{O}^{+}(2,2 ; \mathbb{Z}) \backslash \mathrm{O}^{+}(2,2 ; \mathbb{R}) / \mathrm{SO}(2) \times O(2)$ corresponds to imposing the structure of an elliptic fibration with section on the Calabi-Yau 3 -fold $X$ of the type IIA side [MV96a]. Since $X$ is also $K 3$-fibered, altogether we obtain an elliptic fibration of $X$ over a rational surface $Z \rightarrow \mathbb{C P}^{1}$. In the process of passing to the F-theory limit, the parameter for the size of the fiber of $Z \rightarrow \mathbb{C P}^{1}$ is lost. Moreover, the size of the elliptic fiber in $X \rightarrow Z$ stabilizes to a constant value, which a priori can be taken to be zero. This means that $X$ becomes singular: The Kähler class of $X$ belongs to a face of a Kähler cone.

Since $Z$ is a rational surface, it possesses two special sections, the section at infinity and the zero section. According to [MV96a, MV96b, BIK ${ }^{+}$96] the Kodaira type of the generic fiber over these special sections gives the two factors $H^{1}, H^{2}$ of the unbroken gauge group corresponding to the two heterotic $E_{8}$-bundles in the dual theory. This is compatible with the discussion of (4.1.4): If for $Z \rightarrow \mathbb{C P}^{1}$ we view $z_{1}$ as affine coordinate on the fiber, while $z_{2}$ gives a coordinate on the base, then the zero section is located at $z_{1}=0$, where the behavior of the elliptic fibration $X \rightarrow Z$ is encoded in the polynomials $f^{(0)}, \ldots, f^{(3)}, g^{(0)}, \ldots, g^{(5)}$, and analogously for the section at infinity located at $\left(z_{1}\right)^{-1}=0$ with $f^{(5)}, \ldots, f^{(8)}, g^{(7)}, \ldots, g^{(12)}$ - the bundle parameters as claimed. Under the assumption that the heterotic $K 3$ surface is smooth one can check that no other families of degenerate fibers of non-trivial ADE type occur in the fibration. In particular,

$$
h^{1,1}(X)=1+\operatorname{rk}\left(H^{1}\right)+\operatorname{rk}\left(H^{2}\right)+\rho(Z)
$$

with $\rho(Z)$ the Picard number of the base $Z$. Note that if the base $Z$ of the fibration $X \rightarrow Z$ is minimal, then $Z$ is a Hirzebruch surface $\mathbb{F}_{n}$, and in this case its Picard number is $\rho(Z)=2$. In general, introduce $l \in \mathbb{N}$ such that

$$
\rho(Z)=2+l
$$

and observe from Conjecture 4.1 that $l$ corresponds to the number of pointlike instantons on the heterotic side.

Altogether we have:

Conjecture 4.2. Consider the decompactification limit onto $K 3$ of a heterotic $E_{8} \times E_{8}$ theory on $T^{2} \times K 3$ with smooth $K 3$ surface, unbroken gauge group $H$, and l distinct pointlike instantons. This is a six-dimensional theory with massless spectrum according to Proposition 2.2. Under heterotic - F-theory duality this theory is mapped to the F-theory limit of type IIA strings on a Calabi-Yau 3-fold $X$ with the following properties:

$X$ is elliptically fibered with a section over a rational surface $Z$ with Picard number $\rho(Z)$ and $K 3$-fibered over the base of $Z \rightarrow \mathbb{C P}^{1}$. The fibers of $X \rightarrow Z$ are shrunken to zero volume, corresponding to the size of the heterotic $T^{2}$ becoming infinite. Similarly, the moduli of the fiber of $Z \rightarrow \mathbb{C P}^{1}$ have dropped out, matching the loss of the complex structure parameters for the heterotic $T^{2}$. The Kähler parameter giving the size of the base of $Z \rightarrow \mathbb{C P}^{1}$ corresponds to the scalar dilaton in a tensormultiplet of the heterotic theory. Additional l tensormultiplets accounting for the $l$ pointlike instantons on the heterotic side match the $l$ remaining Kähler parameters of $Z$. Of the heterotic vectormultiplets, $\mathrm{rk}(H)$ account for bundle parameters of the gauge bundle on $T^{2}$ in the original four-dimensional heterotic theory. These are recovered as Kähler moduli of $X$ in terms of degenerate fibers of the elliptic fibration over the two special 
sections of $Z$. Namely, the generic fibers over these sections are ADE type Kodaira fibers which match the ADE types of the summands of the gauge algebra $\mathfrak{h}$. Altogether we have

$$
\rho(Z)=l+2, \quad h^{1,1}(X)=3+\operatorname{rk}(H)+l .
$$

Finally, the $n_{H}^{0}$ neutral hypermultiplets of the heterotic theory match the hypermultiplets that are carried unaltered from the type IIA theory into the F-theory limit:

$$
n_{H}^{0}=h^{1,2}(X)+1 \text {. }
$$

So far, we have deliberately omitted a discussion of charged hypermultiplets on the F-theory side, although the conjectured heterotic - F-theory duality predicts their existence. Indeed, since delicate and interesting issues arise from their investigation we devote much of the Section 5.2 to their study.

The decompactification limit of our heterotic theory on $T^{2} \times K 3$ gives a six-dimensional theory. Hence anomaly cancellation (3.2.3) is an issue. In fact, since the numbers of the various supermultiplets in this theory are related to the geometric invariants of the dual Calabi-Yau 3 -fold by Conjecture 4.2, the duality predicts a classically unknown relation between these invariants:

Conjecture 4.3. Let $X \rightarrow Z$ denote an elliptically fibered Calabi-Yau 3-fold with section, where $Z$ is a rational surface. Assume that $X$ gives the background of an F-theory limit of type IIA string theory which is dual to a consistent, that is an anomaly free decompactification limit of heterotic strings on some $T^{2} \times K 3$ to K3. Then there exists an associated gauge group $H$ which arises from families of ADE-type Kodaira fibers in the fibration $X \rightarrow Z$, and a number $n_{H}^{c h}$ of "charged hypermultiplets" which is a "charged dimension"

$$
\operatorname{dim}_{c h}(\varrho)=\operatorname{dim}(\varrho)-\operatorname{dim}\left(\operatorname{ker} \varrho_{\mid} \operatorname{Cartan} \operatorname{torus(H)}\right)
$$

of a representation $\varrho$ of $H$. Moreover, the Picard number $\rho(Z)$ and the Hodge number $h^{1,2}(X)$ obey

$$
h^{1,2}(X)+29 \rho(Z)-\operatorname{dim}(H)+n_{H}^{c h}=301 .
$$

In the above conjecture we have made no assumptions to the effect that $X$ is smooth or that the respective four-dimensional string theories arise from compactification. Indeed, the conjecture is supposed to hold in great generality. In the setting of Conjecture 4.2 and Proposition 2.2 we can prove the conjecture with little difficulty: We know that the invariants $\rho(Z), h^{1,2}(X), n_{H}^{\text {ch }}$ and $H$ are related to the numbers $n_{T}, n_{H}, n_{V}$ of tensor-, hyper- and vectormultiplets as follows:

$$
n_{T}=l+1=\rho(Z)-1, \quad n_{H}=n_{H}^{0}+n_{H}^{c h}=h^{1,2}(X)+1+n_{H}^{c h}, \quad n_{V}=\operatorname{dim}(H) .
$$

Hence the anomaly cancellation condition (3.2.3) implies

$$
301=28+n_{H}-n_{V}+29 n_{T}=h^{1,2}(X)+n_{H}^{c h}-\operatorname{dim}(H)+29 \rho(Z),
$$

as claimed. 
Although the four-dimensional heterotic - type IIA duality as well as the heterotic - F-theory duality remain highly non-trivial conjectures, Conjecture 4.3 has valuable predictive power: On the one hand it serves as an important test for the duality. On the other hand, we so far had to assume that the heterotic $K 3$ surface is smooth, since techniques for a direct investigation of the degeneration phenomena which occur when pointlike instantons coalesce at singularities of the $K 3$ surface are not known. However, using the F-theory dual of such a degeneration much more can be said, in particular the parameters entering Conjecture 4.3 can be calculated directly in the F-theory picture [AM97], see also Section 5.3.7.

\section{$5 \quad$ F-theory on elliptically fibered Calabi-Yau 3-folds}

In this section we explore the geometric setting of the F-theory side in Conjecture 4.2 intrinsically. We discuss how the invariants $h^{1,2}(X), \rho(Z)$ as well as $H$ and $n_{H}^{c h}$, which are related to one another in Conjecture 4.3, should be encoded in the very geometry of an elliptically fibered Calabi-Yau 3-fold $X \rightarrow Z$ with section. The duality predicts the form of the elliptic fibration. For simplicity in this section we assume that the fibration has precisely one section, because this is the situation assumed in several steps of [GM03], which we use severely. It should not be too hard to include cases where the Mordell-Weil group has non-zero rank, but we have not found a full account in the literature, and we have not yet completed the relevant calculations. While $h^{1,2}(X), \rho(Z)$ as well as $H$ can indeed be obtained by a classical analysis, the invariant $n_{H}^{c h}$ remains rather mysterious. We discuss these quantities separately, where we deal with the "classical" analysis in Section 5.1 and with "charged matter" in Section 5.2.

\subsection{Some invariants of elliptically fibered Calabi-Yau 3-folds}

As mentioned above, in many examples a parameter count in (4.1.4) yields a prediction for the value of $h^{1,2}(X)$ in the F-theory picture. This however does not always give the right answer, because deformations of the complex structure of our Calabi-Yau 3-fold need not all be given in terms of polynomial deformations of the singular Weierstraß form. On the other hand, there are classical geometric methods to calculate $h^{1,2}(X)$ from the data predicted by the duality.

Of the invariants involved in Conjecture 4.3, from the fibration $X \rightarrow Z$ one can directly read an associated "gauge group" $H$; this is a semisimple Lie group whose simple factors are in 1: 1 correspondence to families of degenerate fibers in $X \rightarrow Z$ of ADE Kodaira type and such that the ADE types match [Wit96a, AKM00]. Note that indeed according to [Mir83, §7] near smooth points of the reduced discriminant locus of our fibration such families are locally trivializable. Particular care has to be taken with fibers of types $I_{k}, I V, I_{k}^{*}$, and $I V^{*}$, because generically a family of such fibers in $\pi: X \rightarrow Z$, i.e. $\pi^{-1}\left(\Delta_{i}\right)$ for some divisor $\Delta_{i} \subset Z$, is not globally trivializable, because the fibration has monodromy [AG96]. While without monodromy one would associate factors of the gauge algebra according to

$$
I_{k} \mapsto \mathfrak{s u}(k)=\mathfrak{a}_{k-1}, \quad I V \mapsto \mathfrak{s u}(3)=\mathfrak{a}_{2}, \quad I_{k}^{*} \mapsto \mathfrak{s o}(2 k+8)=\mathfrak{d}_{k+4}, \quad I V^{*} \mapsto \mathfrak{e}_{6}
$$

to such families, monodromy can reduce the associated Lie algebra to the corresponding nonsimply-laced algebra that is read from the respective Dynkin diagram under modding out of an 
outer automorphism:

$$
\begin{aligned}
I_{2 k} \mapsto \mathfrak{s p}(k) & =\mathfrak{c}_{k}, \quad I_{2 k+1} \mapsto \mathfrak{s p}(k)=\mathfrak{c}_{k}, \quad I V \mapsto \mathfrak{s p}(1)=\mathfrak{a}_{1}, \\
I_{k}^{*} \mapsto \mathfrak{s o}(2 k+7) & =\mathfrak{b}_{k+3} \quad \text { or, if } k=0, \quad \mathfrak{g}_{2}, \quad I V^{*} \mapsto \mathfrak{f}_{4} .
\end{aligned}
$$

In calculations, the difference between families with and without monodromy cannot be seen in the "short" Weierstraß form (4.1.4). Rather, one needs a "long" Weierstraß form

$$
y^{2}+a_{1}\left(z_{1}, z_{2}\right) x y+a_{3}\left(z_{1}, z_{2}\right) y=x^{3}+a_{2}\left(z_{1}, z_{2}\right) x^{2}+a_{4}\left(z_{1}, z_{2}\right) x+a_{6}\left(z_{1}, z_{2}\right),
$$

where as before $x, y$ are affine coordinates of $\mathbb{C P}^{2}$ for the fiber and $z_{1}, z_{2}$ are appropriate local coordinates of the base $Z$ of $X \rightarrow Z$. Here it is convenient to assume that the divisor $\Delta_{i}$ over which we want to study a family of degenerate fibers is given by $z_{1}=$ const. Then the vanishing orders $\gamma_{1}, \gamma_{2}, \gamma_{3}, \gamma_{4}, \gamma_{6}$ of $a_{1}, a_{2}, a_{3}, a_{4}, a_{6}$ with respect to $z_{2}$ encode the type of generic fiber over $\Delta_{i}$, including information about the monodromy. For example, vanishing orders $\left(\gamma_{1}, \gamma_{2}, \gamma_{3}, \gamma_{4}, \gamma_{6}\right)=(1,2,2,3,5)$ give fibers of type $I V^{*}$ without monodromy, while $\left(\gamma_{1}, \gamma_{2}, \gamma_{3}, \gamma_{4}, \gamma_{6}\right)=(1,2,2,3,4)$ gives type $I V^{*}$ fibers with monodromy. All relevant data are tabulated in [GM03, Table 1].

With $H$ the total gauge group obtained from families of degenerate fibers one has

$$
h^{1,1}(X)=1+\operatorname{rk}(H)+\rho(Z),
$$

and all these data can be read off from the geometry as predicted by the duality. Therefore, to determine the remaining classical invariant $h^{1,2}(X)$ of Conjecture 4.3 we can equivalently compute the Euler characteristic of $X$,

$$
\chi(X)=2\left(h^{1,1}(X)-h^{1,2}(X)\right) .
$$

The rest of this section is devoted to describing an algorithm for the computation of $\chi(X)$.

If $X$ is a Calabi-Yau 3-fold which is elliptically fibered over a rational surface $Z$ and $K 3$-fibered with a section over the base of $Z \rightarrow \mathbb{C P}^{1}$, then it arises from a singular Weierstraß fibration $\widetilde{X} \rightarrow \widetilde{Z}$ over a Hirzebruch surface $\widetilde{Z}=\mathbb{F}_{n}$ by a sequence of blowups in the base. Let us first collect some properties of this singular fibration. As explained above, the fibration over the section $C_{0}$ of $\mathbb{F}_{n}$ at infinity and over the zero section $C_{\infty}{ }^{4}$ is governed by the bundle data of the dual heterotic theory. Particularly, $n=-C_{0}^{2}=C_{\infty}^{2}$ is related to the topological data $k^{1}, k^{2}, l^{1}, l^{2}$ of the two heterotic $E_{8}$ bundles by

$$
k^{1}+l^{1}=12-n, \quad k^{2}+l^{2}=12+n
$$

if the $i^{\text {th }}$ bundle acquires $l^{i}$ pointlike instantons. These identities arise from a number of conjectures in the physics literature [SW96, Wit96c, MV96a], and we view their validity as part of the conjectured duality. Denote by

$$
y^{2}=x^{3}+a\left(z_{1}, z_{2}\right) x+b\left(z_{1}, z_{2}\right)
$$

\footnotetext{
${ }^{4}$ We apologize for this seemingly confusing notation, which however is compatible with [AM97] and thus facilitates a comparison to that work. See [GH78, p. 518] for the standard mathematical notations.
} 
the singular Weierstraß fibration $\widetilde{X} \rightarrow \widetilde{Z}$ with $\widetilde{Z}=\mathbb{F}_{n}$, with $z_{1}, z_{2}$ affine coordinates on the zero section $C_{\infty}$ and respectively the fiber $F$ of $\mathbb{F}_{n}$. Moreover $a\left(z_{1}, z_{2}\right)$ and $b\left(z_{1}, z_{2}\right)$ are polynomials as in (4.1.4) which define divisors $\widetilde{A}$ and $\widetilde{B}$ in $\widetilde{Z}$. The fibers of (5.1.4) degenerate over the discriminant $\widetilde{\Delta} \subset \widetilde{Z}$ which hence captures all the interesting topology of $\widetilde{X}$ and eventually of $X$. The discriminant is the zero locus of $\delta=4 a^{3}+27 b^{2}$. Since $\widetilde{X} \rightarrow \widetilde{Z}$ is assumed to be a singular Calabi-Yau 3-fold, $\widetilde{X}$ must in particular have trivial canonical class. With $L=-K_{\widetilde{Z}}$ denoting the anticanonical class of $\widetilde{Z}=\mathbb{F}_{n}$ this implies (see e.g. [Asp97, §6.2])

$$
\widetilde{\Delta}=3 \widetilde{A}=2 \widetilde{B} \quad \text { with } \widetilde{A}=4 L, \quad \widetilde{B}=6 L .
$$

Recall also

$$
L=2 C_{0}+(2+n) F, \quad C_{0}^{2}=-n, \quad F^{2}=0, \quad C_{0} \cdot F=1, \quad C_{\infty}=C_{0}+n F, \quad C_{\infty}^{2}=n .
$$

In the context of our duality the discriminant $\widetilde{\Delta}$ in general decomposes into several irreducible components,

$$
\widetilde{\Delta}=\widetilde{\Delta}_{h e t}+\widetilde{\Delta}^{\prime}
$$

where the generic fibers over the irreducible $\widetilde{\Delta}^{\prime}$ are of type $I_{1}$, while $\widetilde{\Delta}_{\text {het }}$ is in general reducible and in particular accounts for more exotic families of degenerate fibers dictated by the heterotic dual. $\widetilde{\Delta}_{h e t}$ in the present context always consists of a collection of smooth rational curves in $\widetilde{Z}=\mathbb{F}_{n}$.

As mentioned above, the Calabi-Yau 3-fold $X \rightarrow Z$ is obtained by a sequence of blowups of $\widetilde{Z}$ from (5.1.4) to yield $Z$. We always assume that sufficiently many blowups have been performed such that all fibers of $X \rightarrow Z$ are minimal according to Kodaira's list of degenerate fibers [Kod64]. As we shall see when we present some examples in Section 5.3, the blowups can be a delicate issue which needs to be dealt with by a detailed analysis of the singularities in (5.1.2). For the proper transforms of the various divisors we write

$$
A=A_{\text {het }}+A^{\prime}, \quad B=B_{\text {het }}+B^{\prime}, \quad \text { and then } \quad \Delta=\Delta_{\text {het }}+\Delta^{\prime},
$$

where in particular $\Delta^{\prime}$ is the proper transform of $\widetilde{\Delta}^{\prime}$. For later bookkeeping let us denote by $b_{j}$ the number of blowups of points on $\widetilde{\Delta}^{\prime}$ of multiplicity $\alpha_{j}$. In particular,

$$
\Delta^{\prime}\left(\Delta^{\prime}+K_{Z}\right)=\widetilde{\Delta}_{\widetilde{Z}}^{\prime}\left(\widetilde{\Delta}_{\widetilde{Z}}^{\prime}+K_{\widetilde{Z}}\right)-\sum_{j} \alpha_{j}\left(\alpha_{j}-1\right) b_{j}
$$

see e.g. [GM03, Corollary 6.3].

All contributions to the Euler characteristic $\chi(X)$ are captured by the discriminant $\Delta \subset Z$ of $X \rightarrow Z$ and the singular fibers over it. To keep track of all of them, we consider the decomposition of $\Delta$ into irreducible components, $\Delta=\bigcup_{i=1}^{r} \Delta_{i} \bigcup \Delta^{\prime}$, where from the above all $\Delta_{i}$ are smooth rational curves and the generic fibers over $\Delta^{\prime}$ are of Kodaira type $I_{1}$. Contributions to $\chi(X)$ can come from the generic fibers over each component of $\Delta$, from intersection points between any two of these components, from singular points (cusps) on $\Delta^{\prime}$, and from the Euler characteristic of $\Delta$ itself. See [Mir83, (3.1)] for confirmation that we may indeed assume that no other singularities but cusps occur in the residual discriminant $\Delta^{\prime}$. To tabulate all this information, we need to introduce some notation. 
First, let $\chi_{i} \in \mathbb{N}$ denote the Euler characteristic of the generic fibers over $\Delta_{i}$. The values of $\chi_{i}$ in each case are tabulated in [GM03, Table 3] in the column marked "m". Note that the corresponding number for $\Delta^{\prime}$ is 1 . By $P^{1}, \ldots, P^{I}$ we denote all intersection points of irreducible components of $\Delta$. Particularly let $I^{\prime}$ denote the number of points $P^{i}$ on $\Delta^{\prime}$. When counting intersection points of two given components of $\Delta$ care has to be taken since the intersection number of the respective divisors counts points with multiplicities, while our $P^{1}, \ldots, P^{I}$ are understood to be pairwise distinct. Since all $\Delta_{i}$ are assumed to be smooth rational curves, this issue is only relevant in interpreting $\Delta_{i} \cdot \Delta^{\prime}$. The necessary local case by case analysis has been carried out in [GM03], and the respective multiplicities are found in [GM03, Table 2 ] as exponents of $t$ in the columns marked "l.e. at $P_{k}$ ", and where "transversal" amounts to multiplicity 1. Note that in several cases intersections of $\Delta_{i}$ and $\Delta^{\prime}$ come in two different "types $P_{1}, P_{2}$ ", meaning that for $P \in \Delta_{i} \cap \Delta^{\prime}$ the local geometry near $\pi^{-1}(P)$ depends on whether $P$ is of type $P_{1}$ or $P_{2}$. A case by case analysis of the defining polynomials shows that there are never more than two types. One can use [GM03, Table E] to determine the numbers $B_{1}, B_{2}$ of each type of intersection and thus to disentangle the value of $\Delta_{i} \cdot \Delta^{\prime}$ in these cases. Moreover,

$$
-\varepsilon_{1} B_{1}-\varepsilon_{2} B_{2}
$$

with $\varepsilon_{i}$ taken from [GM03, Table 4] gives the contribution to $I^{\prime}$ from the collision $\Delta_{i} \cap \Delta^{\prime}$. The Euler characteristic of each fiber $\pi^{-1}\left(P^{i}\right)$ can be found in [GM03, Table 4] if $P^{i} \in \Delta^{\prime}$, and otherwise one uses [Mir83, Table (14.1)]. Even though in the latter work, Miranda does not take monodromy into account, these calculations are still valid for our purposes, since the computation of the Euler characteristic only depends on the geometry of the fiber over $P_{i}$ which he describes in great detail.

Cusps of $\Delta^{\prime}$ are denoted by $Q^{1}, \ldots, Q^{C}$, and each of them carries a special fiber of type $I I$, contributing $\chi\left(\pi^{-1}\left(Q^{i}\right)\right)=2$ to the Euler characteristic. Note that cusps in (5.1.4) are characterized by the simultaneous vanishing of $a$ and $b$, such that their total number in the resolved Calabi-Yau 3 -fold $X$ is given by $A^{\prime} \cdot B^{\prime}$, as long as these cusps do not coalesce with an intersection point of $\Delta^{\prime}$ with one of the other components of $\Delta$. From [GM03, Proposition 8] one obtains the general formula for the number $C$ of cusps, where an overcounting in $A^{\prime} \cdot B^{\prime}$ is observed when $\Delta^{\prime}$ intersects $\Delta_{i}$ which carry generic fibers of types $I_{k}, I_{0}^{*}, I_{1}^{*}$, or $I_{2}^{*}$. One uses [GM03, Table 4] to read off the invariants entering here: If $\Delta^{\prime} \cap \Delta_{i}$ has $B_{1}$ intersection points of "type $P_{1}$ " and $B_{2}$ intersection points of "type $P_{2}$ ", then

$$
C=A^{\prime} \cdot B^{\prime}-\mu_{1} B_{1}-\mu_{2} B_{2}
$$

with $\mu_{1}, \mu_{2}$ taken from [GM03, Table 4].

Altogether we have

$$
\begin{aligned}
\chi(X)=\chi & \left(\pi^{-1}\left(\Delta^{\prime}-\bigcup_{j=1}^{I}\left\{P^{j}\right\}-\bigcup_{j=1}^{C}\left\{Q^{j}\right\}\right)\right)+\sum_{i=1}^{r} \chi\left(\Delta_{i}-\bigcup_{j=1}^{I}\left\{P^{j}\right\}\right) \chi_{i} \\
& +\sum_{j=1}^{I} \chi\left(\pi^{-1}\left(P^{j}\right)\right)+2 C .
\end{aligned}
$$


From the above discussion we can determine all contributions, where the first one simplifies to

$$
\begin{aligned}
& \begin{aligned}
\chi\left(\pi^{-1}\left(\Delta^{\prime}-\bigcup_{j=1}^{I}\left\{P^{j}\right\}-\bigcup_{j=1}^{C}\left\{Q^{j}\right\}\right)\right) & =\chi\left(\pi^{-1}\left(\Delta^{\prime}\right)\right)-I^{\prime}-C \\
& =-\Delta^{\prime}\left(\Delta^{\prime}+K_{Z}\right)+2 C-I^{\prime}-C
\end{aligned} \\
& \stackrel{(5.1 .7)}{=}-\widetilde{\Delta}^{\prime}\left(\widetilde{\Delta}^{\prime}+K_{\widetilde{Z}}\right)+\sum_{j} \alpha_{j}\left(\alpha_{j}-1\right) b_{j}-I^{\prime}+C
\end{aligned}
$$

In summary,

Proposition 5.1. Let $X \rightarrow Z$ be a Calabi-Yau 3-fold which arises via a sequence of blowups in the base of the singular Weierstraß fibration $\widetilde{X} \rightarrow \widetilde{Z}$ over a Hirzebruch surface $\widetilde{Z}=\mathbb{F}_{n}$, associated to the data coming from a heterotic $E_{8} \times E_{8}$ theory compactified on a K3 surface. Assume that $X \rightarrow Z$ has precisely one section and that all fibers are minimal. Then the Euler characteristic of $X$ is given by

$$
\begin{aligned}
\chi(X)= & -\widetilde{\Delta}^{\prime}\left(\widetilde{\Delta}^{\prime}+K_{\widetilde{Z}}\right)+\sum_{j} \alpha_{j}\left(\alpha_{j}-1\right) b_{j} \\
& +\sum_{i=1}^{r} \chi\left(\Delta_{i}-\bigcup_{j=1}^{I}\left\{P^{j}\right\}\right) \chi_{i}+\sum_{j=1}^{I} \chi\left(\pi^{-1}\left(P^{j}\right)\right)-I^{\prime}+3 C,
\end{aligned}
$$

where generically $C=A^{\prime} \cdot B^{\prime}$, but in general $C$ is obtained from (5.1.9) and $I^{\prime}$ is obtained according to the discussion around (5.1.8).

\subsection{Charged matter}

Above we have explained that the heterotic - F-theory duality yields sufficiently detailed predictions about the elliptically fibered Calabi-Yau 3-fold $X \rightarrow Z$ on the F-theory side such that one can recover geometric invariants like $h^{1,2}(X)$ and $h^{1,1}(X)$ along with $\rho(Z)$ and an associated "gauge group" $H$. In light of Conjecture 4.3 one could then simply define

$$
n_{H}^{c h}:=301+\operatorname{dim}(H)-h^{1,2}(X)-29 \rho(Z)
$$

and accept it as a new invariant of elliptically fibered Calabi-Yau 3-folds with section that occur as F-theory duals of well-defined heterotic string theories. However, from the derivation on the heterotic side for this invariant around Proposition 2.2, we know that $n_{H}^{c h}$ is a purely gauge theoretic quantity obtained through the representation theory of $H$. Hence rather than using Conjecture 4.3 as a definition of $n_{H}^{c h}$ one should expect an intrinsic geometric interpretation of this quantity and view the anomaly cancellation condition as a classically unknown relation between geometric invariants attached to elliptically fibered Calabi-Yau 3-folds with section. This is the viewpoint we are going to take here.

In fact, if the heterotic - F-theory duality holds on the level of string theory, then we are forced into this viewpoint: According to Proposition 2.2 the quantity $n_{H}^{c h}$ accounts for part of the hypermultiplet spectrum of the dual heterotic theory, namely the charged part, and hence must also occur on the F-theory side. Similarly, for non-Abelian $H$ we have $\operatorname{dim} H-\operatorname{rk} H$ 
additional vectormultiplets to account for which so far have escaped our explanations on the F-theory side.

Since in the $F$-theory limit the fibers of the elliptic fibration shrink to zero size, i.e. the CalabiYau 3-fold $X$ is taken to the boundary of the Kähler cone, one can expect the additional multiplets to arise due to this degeneration of $X$. Indeed, explanations along these lines can be found in the literature, see in particular [KV97, AKM00]. This work connects the appearance of additional multiplets to two phenomena which occur in this limit, distinguished by the way the fibers of the elliptic fibration degenerate: (1) in families, or (2) isolated. Let us attempt to summarize and comment on these explanations.

\subsubsection{Families of degenerate fibers yielding new vectormultiplets}

From Proposition 2.4 we know that on a smooth Calabi-Yau 3-fold $X$ a type IIA string theory possesses $h^{1,1}(X)$ vectormultiplets. These in particular include an RR three-form $C_{\mu i j}$ in each multiplet which can be integrated over two-cycles in $X$ to produce one-forms. The latter can be interpreted as analogs of Yang-Mills connection one-forms of a gauge theory which can become non-Abelian when $(-2)$ curves in $X$ shrink to zero size [AKM00].

More precisely, consider a family of degenerate elliptic fibers in an elliptically fibered CalabiYau 3-fold such that the degenerate fiber is a bouquet of rational curves. As explained above, to this type of family one associates a simple factor $G_{i}$ of the "gauge group" $H$, an ADE type Lie group. Each rational curve in the bouquet has normal bundle $\mathcal{O} \oplus \mathcal{O}(-2)$ in $X$, and in the family of degenerate fibers it sweeps out a four-cycle $S_{i}$ in $X$ with normal bundle $\mathcal{O}(-2)$. These four-cycles are in 1: 1 correspondence with the generators of a Cartan torus of $G_{i}$. Indeed, following [Wit96a, KV97, AKM00] one defines $U(1)$ charges with respect to each $S_{i}$ on any $(-2)$ curve in the degenerate fiber via the intersection form. Here one views the $(-2)$ curve as the cycle which a two-brane wraps to produce a new massless particle with "electric charge" given by its $U(1)$ charge if the size of the relevant $(-2)$ curve shrinks to zero. Since the $(-2)$ curves in the degenerate fiber are in 1: 1 correspondence with the roots of $G_{i}$, and because the rational curves underlying $S_{i}$ have intersection form given by the Cartan matrix of $G_{i}$, one obtains the adjoint representation of $G_{i}$. In particular, the "gauge representation" associated to the $S_{i}$ is enhanced from a $\operatorname{rk} G_{i}$ to a $\operatorname{dim} G_{i}$ dimensional representation, accounting for the missing vectormultiplets: One has to add one "charged" vectormultiplet corresponding to each root of $G_{i}$.

In fact, for definiteness recall from Section 5.1 that

$$
\Delta=\bigcup_{i=1}^{r} \Delta_{i} \cup \Delta^{\prime},
$$

with smooth irreducible curves $\Delta_{i}$, and $\Delta^{\prime}$ the residual discriminant of the fibration $\pi: X \rightarrow Z$, an irreducible curve over which the generic fibers are of Kodaira type $I_{1}$. For each $\Delta_{i}$ the fiber over it gives rise to a simply-laced Lie algebra, which may be trivial if the fibers are of type $I_{1}$ or $I I$. If there is no monodromy within $\pi^{-1}\left(\Delta_{i}\right)$, then this determines the associated factor $G_{i}$ of $H$. If within $\pi^{-1}\left(\Delta_{i}\right)$ there is monodromy, then $G_{i}$ is the corresponding non-simply-laced Lie group which descends from the simply laced one as listed on the algebra level in (5.1.1). To 
summarize, the enhanced gauge group is

$$
H=\prod_{i=1}^{r} G_{i}
$$

and the total number of vector multiplets is $\operatorname{dim} H=\sum_{i=1}^{r} \operatorname{dim} G_{i}$.

\subsubsection{Colliding degenerate fibers yielding charged hypermultiplets}

We have now accounted for all effects of the degeneration of $X$ in the F-theory limit, apart from the special degenerate fibers which occur over collision points of irreducible components of the discriminant $\Delta \subset Z$ of the fibration $\pi: X \rightarrow Z$. It is important to note that the type of fiber over such a collision point is not simply obtained by adding the vanishing orders of the polynomials $a_{1}, a_{2}, a_{3}, a_{4}, a_{6}$ in (5.1.2). Rather, according to [Mir83] a generic curve $C \subset Z$ through the collision point yields a surface $\pi^{-1}(C)$ with a singularity at the collision point. Minimally resolving this singularity gives a degenerate fiber $Y$ of the type predicted by simply adding the vanishing orders of $a_{1}, \ldots, a_{6}$. The isolated degenerate fiber of $\pi: X \rightarrow Z$ is hence obtained by contracting some of the irreducible curves in $Y$. The explanations in [KV97] amount to translating this result into the language of the "gauge theory" associated to the group $H$ as described above. The geometric setting hints towards various interesting phenomena that are related to these collision points. Particularly in view of the gauge theory attached to families of degenerate fibers according to Section 5.2.1, one expects representations of $\mathfrak{g}_{i} \oplus \mathfrak{g}_{j}$ attached to the fibers over collision points which are non-trivial with respect to both summands, where $\mathfrak{g}_{i}, \mathfrak{g}_{j}$ are the gauge algebras associated to the two colliding families of degenerate fibers. This phenomenon is the expected origin of the charged hypermultiplets [Wit96a, KV97, AKM00].

For the geometry of elliptically fibered Calabi-Yau 3-folds $X \rightarrow Z$ which occur in the heterotic - F-theory duality this means that at least formally it should be possible to associate to each degenerate fiber over an intersection of irreducible components of the discriminant, (a) a representation of the total gauge group $H$, and (b) a prescription to calculate its charged dimension, such that $n_{H}^{c h}$ is the sum of all these charged dimensions. For the situation where $\Delta=\cup_{i=1}^{r} \Delta_{i} \cup \Delta^{\prime}$ obeys $\Delta_{i} \cap \Delta_{j}=\emptyset$ for all $i \neq j$ this idea has been carried out in [GM03]. This work hence covers all F-theory duals of heterotic theories compactified on smooth $K 3$ surfaces and with smooth bundles. To calculate $n_{H}^{c h}$ from the results of [GM03] one proceeds as follows: We have already explained in Section 5.1 how to determine the number of intersection points $P^{1}, \ldots, P^{I}$ ( $I=I^{\prime}$ such that one of the colliding divisors is always $\Delta^{\prime}$ under our assumptions), along with their multiplicities and the information whether or not monodromy is involved in one of the colliding families. While [GM03, Table 4] gives the type of fiber over each collision point, the associated representations are listed in [GM03, Table A]. Here, $\rho_{1}, \rho_{2}$ denote representations attached to collision points of "type $P_{1}, P_{2}$ " respectively (see Section 5.1 for this terminology), and $\rho_{0}$ is a representation which is "non-isolated" in the sense that monodromy prevents a localization over the collision points.

While representations of type $\rho_{1}, \rho_{2}$ contribute to $n_{H}^{c h}$ according to their dimensions at each collision point, greater care has to be taken when determining the contributions for representations of type $\rho_{0}$. First, if at a collision point $P^{k} \in \Delta_{i} \cap \Delta^{\prime}$ monodromy occurs, then one needs to 
work with a branched cover $\Delta_{i}^{\prime}$ of $\Delta_{i}$ which parametrizes the exceptional curves in one homology class [GM03, Corollary 1.3]. The difference $g_{i}^{\prime}-g_{i}$ of genera between $\Delta_{i}^{\prime}$ and $\Delta_{i}$ is obtained from [GM03, Tabel E] and replaces the number of collision points in $\Delta_{i} \cap \Delta^{\prime}$ in the contribution to $n_{H}^{c h}$ from $\rho_{0}$ [GM03, Theorem 8.2]. This is an effect of having "non-isolated" representations: Some of the points in $\Delta_{i} \cap \Delta^{\prime}$ belong to the same orbit under this representation. There is a second crucial effect of monodromy. Recall that we distinguish between charged and uncharged hypermultiplets, where uncharged matter is characterized by the fact that it transforms trivially under the gauge group. Generically, on the heterotic side the representations $L_{a}^{i} \otimes Q_{a}^{i}$ in (2.2.7) with non-trivial $L_{a}^{i}, Q_{a}^{i}$ yield trivial kernel when restricted to the Cartan torus $\mathfrak{t}$ of the gauge group $H$. However, with monodromy this need not be the case such that one can obtain additional contributions to the uncharged matter, and accordingly a smaller contribution to the charged matter from $\rho_{0}$ as above. In [GM03] the relevant contribution of $\rho_{0}$ to $n_{H}^{c h}$ is called the charged dimension

$$
\operatorname{dim}\left(\rho_{0}\right)_{c h}:=\operatorname{dim}\left(\rho_{0}\right)-\operatorname{dim} \operatorname{ker}\left(\rho_{0}\right)_{\mid \mathfrak{t}}
$$

and can be obtained from [GM03, Table B].

The invariant $n_{H}^{c h}$ altogether receives a contribution $\left(g_{i}^{\prime}-g_{i}\right) \operatorname{dim}\left(\rho_{0}\right)_{c h}$ from $\rho_{0}$. If $\operatorname{dim}\left(\rho_{0}\right)_{c h} \neq$ $\operatorname{dim}\left(\rho_{0}\right)$ then $n_{H}^{0}$ receives an additional contribution $\left(g_{i}^{\prime}-g_{i}\right)\left(\operatorname{dim}\left(\rho_{0}\right)-\operatorname{dim}\left(\rho_{0}\right)_{c h}\right)$ which must be added to our formula (2.2.23) in order for $h^{1,2}(X)=n_{H}^{0}+1$ to hold in Conjecture 4.2. To our knowledge, this latter correction has escaped mention in the literature, so far.

After having established the prescription for the calculation $n_{H}^{\text {ch }}$ from [GM03] we naturally ask whether the invariants $h^{1,2}(X), \rho(Z), \operatorname{dim}(H)$, and $n_{H}^{c h}$ associated to an elliptic fibration $X \rightarrow Z$ obey the anomaly cancellation condition of Conjecture 4.3. Indeed, one of the main results of [GM03] is the confirmation of this classically unknown identity in the cases they treat. Moreover, a verification of anomaly cancellation in the context of the Green-Schwarz mechanism is given. These results of [GM03] yield yet another striking and highly non-trivial piece of evidence in favor of the conjectured heterotic - F-theory duality. However, they arise from a local case-by-case analysis and do not give an entirely intrinsic explanation for the origin of the representations attached formally to collisions of families of degenerate fibers in $X \rightarrow Z$. Although the familiar branching rules from the heterotic dual are invoked to determine the relevant representations, as is also suggested in [KV97], the actual mathematical origin of the "gauge theory" associated to degenerate fibers remains mysterious.

Indeed, in light of the gauge theory attached to each family of degenerate fibers according to Section 5.2.1, there seems to be a natural explanation in terms of the local geometry of the isolated degenerate fibers which also gives a lead on how to calculate $n_{H}^{c h}$ in general [AKM00]. As mentioned above, one expects that both factors $G_{i}, G_{j}$ of the gauge group associated to two colliding families should act non-trivially on the representation associated to the collision. While generic rational curves in this isolated fiber will have vanishing charge with respect to one of the two groups, in some cases such a curve can have non-trivial charge with respect to both $G_{i}$ and $G_{j}$. As before, this charge is encoded in the respective normal bundle of the rational curve in $X$. Generically, such a curve will have normal bundle $\mathcal{O} \oplus \mathcal{O}(-2)$ or $\mathcal{O}(-2) \oplus \mathcal{O}$, while in some cases curves with normal bundle $\mathcal{O}(-1) \oplus \mathcal{O}(-1)$ can occur. The latter are expected to be the sources of the charged hypermultiplets. Note however that the explanation definitely needs adjustment. In the setting which the authors of [GM03] restrict to, either $G_{i}$ 
or $G_{j}$ is trivial, so the above approach would predict absence of charged hypermultiplets. This prediction is false. We believe that in [AKM00] one needs to assume that neither of $G_{i}, G_{j}$ is trivial.

The above idea is shown to work for colliding families of type $I_{n}$ and $I_{m}$, respectively, in [AKM00], but it seems not to have been pushed further in the physics literature. However, we have found encouraging confirmation beyond this case in [Mir83], where the local geometry of fibers over such collision points is studied in detail. In particular, a list of "fundamental collisions" is given, to which all other collisions can be reduced by appropriate blowups of the base. The topology of the isolated fiber for each fundamental collision is worked out, along with the normal bundles of the irreducible components of these fibers. The only fundamental collisions with at least one irreducible component of the isolated fiber having normal bundle $\mathcal{O}(-1) \oplus \mathcal{O}(-1)$ are collisions of types

$$
I_{n}+I_{m}, \quad I_{n}+I_{m}^{*}, \quad I V+I_{0}^{*}, \quad \text { and } \quad I I I+I_{0}^{*} .
$$

We view it as a striking confirmation of the ideas of [AKM00] that in a wealth of examples where $n_{H}^{c h}$ can be calculated on the heterotic side and hence a prediction for its value is available, its geometric derivation involves only collisions of type (5.2.1), or non-fundamental collisions, or collisions which generically suffer from monodromy - and no counter example is known to us. In other words, for all cases that are covered by [Mir83] the ideas of [AKM00] can be confirmed. Nevertheless - and surprisingly - Miranda's list has not yet been extended to all relevant cases to provide a complete intrinsic explanation for charged hypermultiplets in the geometry of elliptically fibered Calabi-Yau 3-folds. In particular, monodromy is not taken into account in [Mir83] - and in fact is also not addressed in [KV97]. Of course, several further examples are discussed in [AKM00], including some with monodromy, and various methods that apply in special cases are known [KMP96, Wit96a, AG96, BIK ${ }^{+} 96$, Sad96, KV97, IMS97, CPR98, Int98, DE99], but a general intrinsic understanding apparently has not yet been reached. We are currently working on filling this gap.

\subsection{Examples}

In this section we present a number of examples to illustrate the algorithms explained above. They are chosen representatively to show all the special features that to our knowledge can occur in these algorithms.

As a first step, using the heterotic input data we need to specify the respective singular Weierstraß fibration (5.1.2) over $\widetilde{Z}=\mathbb{F}_{n}$ in each case. We assume that the heterotic data $k^{i}, l^{i}, K^{i}, H^{i}$ are given as in Section 3.3. Recall that $n$ is then determined by (5.1.3). Moreover, the fibration $\widetilde{X} \rightarrow \widetilde{Z}$ degenerates over $C_{0}$ and $C_{\infty}$, where $H^{1}, H^{2}$ determine the Kodaira type of the generic singular elliptic fiber. Using [AM97, Table 1] this gives the vanishing orders $\alpha_{i}, \beta_{i}, d_{i}$ of $a, b$, and $\delta$ in the short Weierstraß form (5.1.4) along $C_{0}$ and $C_{\infty}$. Hence we can specify the components $\widetilde{A}^{\prime}, \widetilde{B}^{\prime}, \widetilde{\Delta}^{\prime}$ of the discriminant yielding $A^{\prime}, B^{\prime}, \Delta^{\prime}$ of (5.1.6) after blowup:

$$
\widetilde{A}^{\prime}=4 L-\alpha_{0} C_{0}-\alpha_{\infty} C_{\infty}, \quad \widetilde{B}^{\prime}=6 L-\beta_{0} C_{0}-\beta_{\infty} C_{\infty}, \quad \widetilde{\Delta}^{\prime}=12 L-d_{0} C_{0}-d_{\infty} C_{\infty},
$$

with the notation of (5.1.5). In fact, in most of the cases discussed below no further blowup will be necessary, such that $\widetilde{A}^{\prime}=A^{\prime}, \widetilde{B}^{\prime}=B^{\prime}, \widetilde{\Delta}^{\prime}=\Delta^{\prime}$ and the collision points of components 
of $\Delta$ which take center stage in the analysis are counted by the intersection numbers $\Delta^{\prime} \cdot C_{0}$ and $\Delta^{\prime} \cdot C_{\infty}$. In some cases, though, collisions turn out to be non-minimal, such that blowups are needed, as we shall describe in more detail where necessary.

As mentioned above, to correctly incorporate monodromies instead of the short Weierstraß form (5.1.4) one needs to use the long version (5.1.2) from which the former is obtained via

$$
\begin{aligned}
c_{2}:=a_{1}^{2}+4 a_{2}, \quad c_{4}:=a_{1} a_{3}+2 a_{4}, \quad c_{6}:=a_{3}^{2}+4 a_{6}, \\
a=-\frac{1}{48}\left(c_{2}^{2}-24 c_{4}\right), \quad b=-\frac{1}{864}\left(-c_{2}^{3}+36 c_{2} c_{4}-216 c_{6}\right) .
\end{aligned}
$$

For later convenience let us also introduce the following notation: We write $Y_{\alpha, \beta ; \gamma_{1}, \gamma_{2}, \gamma_{3}, \gamma_{4}, \gamma_{6}}(\mathfrak{g})$ to denote a $Y$-type fiber, where $\alpha, \beta, \gamma_{i}$ are the vanishing orders of $a, b$ in (5.1.4) and $a_{i}$ in (5.1.2), respectively, and $\mathfrak{g}$ is the Lie algebra of the associated gauge group. We sometimes simply call such a fiber "of type $\left(\alpha, \beta ; \gamma_{1}, \gamma_{2}, \gamma_{3}, \gamma_{4}, \gamma_{6}\right)$ ".

\subsubsection{Completely broken gauge group}

If the gauge group is trivial, $H=\{\mathrm{id}\}$, this means that our $K 3$ bundle has holonomy $K=$ $E_{8} \times E_{8}$. Since no additional singular fibers are imposed, we have

$$
\begin{aligned}
A^{\prime} & =4 L=8 C_{0}+(8+4 n) F \\
B^{\prime} & =6 L=12 C_{0}+(12+6 n) F \\
\Delta^{\prime} & =12 L=24 C_{0}+(24+12 n) F .
\end{aligned}
$$

Because there are no collisions, $\widetilde{Z}=Z$ needs no further blowups and hence

$$
\rho(Z)=2, \quad h^{1,1}(X)=3 .
$$

Furthermore we directly obtain all contributions to the formula for $\chi(X)$ in Proposition 5.1:

$$
\begin{array}{crr}
\Delta^{\prime} \cdot\left(\Delta^{\prime}+K_{\widetilde{Z}}\right)=1056, & \sum_{j} \alpha_{j}\left(\alpha_{j}-1\right) b_{j}=0, & \sum_{i=1}^{r} \chi\left(\Delta_{i}-\bigcup_{j=1}^{I}\left\{P^{j}\right\}\right) \chi_{i}=0, \\
\sum_{j=1}^{I} \chi\left(\pi^{-1}\left(P^{j}\right)\right)=0, & I^{\prime}=0, & C=A^{\prime} \cdot B^{\prime}=192 .
\end{array}
$$

Hence

$$
\chi(X)=-480, \quad h^{1,2}(X)=243 .
$$

Moreover since there are no collisions, no charged hypermultiplets occur, $n_{H}^{c h}=0$. First, anomaly cancellation according to Conjecture 4.3 is seen to hold (as of course follows from the results of [GM03]). Second, comparing these data to the ones obtained for the heterotic dual (3.3.1), we see that Conjecture 4.2 is met. 


\subsubsection{Unbroken $E_{8}$ gauge group}

If the gauge group is $H=E_{8} \times\{\mathrm{id}\}$, this means that our heterotic $K 3$ bundle has holonomy $K=\{\mathrm{id}\} \times E_{8}$. So we are imposing $I I^{*}$ fibers on $C_{0}$ and

$$
\begin{aligned}
& \widetilde{A}^{\prime}=4 L-4 C_{0}=4 C_{0}+(8+4 n) F \\
& \widetilde{B}^{\prime}=6 L-5 C_{0}=7 C_{0}+(12+6 n) F \\
& \widetilde{\Delta}^{\prime}=12 L-10 C_{0}=14 C_{0}+(24+12 n) F .
\end{aligned}
$$

We have $\widetilde{\Delta}^{\prime} \cdot C_{0}=2(12-n)$ and find that each intersection has multiplicity $\alpha_{j}=2$. These intersections are non-minimal, so $b_{j}=12-n$ blowups are necessary, yielding

$$
\rho(Z)=14-n, \quad h^{1,1}(X)=23-n .
$$

After blowup, no collisions are left. The contributions to the formula for $\chi(X)$ in Proposition 5.1 are:

$$
\begin{aligned}
\Delta^{\prime} \cdot\left(\Delta^{\prime}+K_{\widetilde{Z}}\right) & =130 n+596, & \sum_{j} \alpha_{j}\left(\alpha_{j}-1\right) b_{j} & =24-2 n, \\
\sum_{i=1}^{r} \chi\left(\Delta_{i}-\bigcup_{j=1}^{I}\left\{P^{j}\right\}\right) \chi_{i} & =20, & \sum_{j=1}^{I} \chi\left(\pi^{-1}\left(P^{j}\right)\right) & =0, \\
I^{\prime} & =0, & C=\widetilde{A}^{\prime} \cdot \widetilde{B}^{\prime} & =104+24 n .
\end{aligned}
$$

Hence

$$
\chi(X)=-60 n-240, \quad h^{1,2}(X)=143+29 n .
$$

Since there are no collisions, no charged hypermultiplets occur, $n_{H}^{c h}=0$. Anomaly cancellation according to Conjecture 4.3 holds (in accord with [GM03]). Moreover, comparing these data to the ones obtained for the heterotic dual (3.3.2) with $l=-n+12$ according to (5.1.3), we see that Conjecture 4.2 is met.

\subsubsection{Unbroken $E_{7}$ gauge group}

If the gauge group is $H=E_{7} \times\{\mathrm{id}\}$, this means that our $K 3$ bundle has holonomy $K=$ $\mathrm{SU}(2) \times E_{8}$. So we are imposing $I I I^{*}$ fibers on $C_{0}$ and

$$
\begin{aligned}
& \widetilde{A^{\prime}}=4 L-3 C_{0}=5 C_{0}+(8+4 n) F, \\
& \widetilde{B}^{\prime}=6 L-5 C_{0}=7 C_{0}+(12+6 n) F, \\
& \widetilde{\Delta}^{\prime}=12 L-9 C_{0}=15 C_{0}+(24+12 n) F .
\end{aligned}
$$

We have $\widetilde{\Delta}^{\prime} \cdot C_{0}=3(8-n)$ and from [GM03, Table 2] find that each intersection has multiplicity $\alpha_{j}=3$. These intersections are minimal, but we can choose to blow up $b_{j}=l$ of them, yielding

$$
\rho(Z)=2+l, \quad h^{1,1}(X)=10+l .
$$


After blowup, $(8-n-l)$ collisions are left. From [GM03, Table 4] the Euler characteristic of the isolated fibers over such collision points is 9 . The contributions to the formula for $\chi(X)$ in Proposition 5.1 hence are:

$$
\begin{aligned}
\Delta^{\prime} \cdot\left(\Delta^{\prime}+K_{\widetilde{Z}}\right) & =126 n+642, & & \sum_{j} \alpha_{j}\left(\alpha_{j}-1\right) b_{j}=6 l, \\
\sum_{i=1}^{r} \chi\left(\Delta_{i}-\bigcup_{j=1}^{I}\left\{P^{j}\right\}\right) \chi_{i} & =-54+9 n+9 l, & \sum_{j=1}^{I} \chi\left(\pi^{-1}\left(P^{j}\right)\right) & =72-9 n-9 l, \\
I^{\prime} & =8-n-l, & C=\widetilde{A}^{\prime} \cdot \widetilde{B}^{\prime}-l & =23 n+116-l .
\end{aligned}
$$

The formula for the number of cusps $C$ takes into account that each collision of $C_{0}$ with $\widetilde{\Delta}^{\prime}$ is also a cusp of $\widetilde{\Delta}^{\prime}$ which is resolved if we blow up. Altogether

$$
\chi(X)=-56 n-284+4 l, \quad h^{1,2}(X)=28 n+152-l .
$$

By [GM03, Table A] each of the $(8-n-l)$ collisions carries charged matter, contributing $\frac{1}{2} \mathbf{5 6}$ to $n_{H}^{c h}$. One hence has $n_{H}^{c h}=224-28 n-28 l$. Anomaly cancellation according to Conjecture 4.3 holds, and comparing these data to the ones obtained for the heterotic dual (3.3.3) with $k^{1}+l=-n+12$ and $k^{2}=n+12$ according to (5.1.3), we see that Conjecture 4.2 is met.

\subsubsection{Unbroken $E_{6}$ gauge group}

If the gauge group is $H=E_{6} \times\{\mathrm{id}\}$, this means that our $K 3$ bundle has holonomy $K=$ $\mathrm{SU}(3) \times E_{8}$. So we are imposing $I V^{*}$ fibers on $C_{0}$, but with trivial monodromy, which is the non-generic case. We have

$$
\begin{aligned}
& \widetilde{A}^{\prime}=4 L-3 C_{0}=5 C_{0}+(8+4 n) F, \\
& \widetilde{B}^{\prime}=6 L-4 C_{0}=8 C_{0}+(12+6 n) F, \\
& \widetilde{\Delta}^{\prime}=12 L-8 C_{0}=16 C_{0}+(24+12 n) F, \quad \widetilde{\Delta}^{\prime} \cdot C_{0}=2(12-2 n)=4(6-n) .
\end{aligned}
$$

From [GM03, Table 2] each intersection between $C_{0}$ and $\widetilde{\Delta}^{\prime}$ has multiplicity $\alpha_{j}=4$. These intersections are minimal, but we can choose to blow up $b_{j}=l$ of them, yielding

$$
\rho(Z)=2+l, \quad h^{1,1}(X)=9+l .
$$

After blowup, $(6-n-l)$ collisions are left. From [GM03, Table 4] the Euler characteristic of the isolated fibers over such collision points is 9 . The contributions to the formula for $\chi(X)$ in Proposition 5.1 hence are:

$$
\begin{aligned}
\Delta^{\prime} \cdot\left(\Delta^{\prime}+K_{\widetilde{Z}}\right) & =120 n+688, & \sum_{j} \alpha_{j}\left(\alpha_{j}-1\right) b_{j} & =12 l, \\
\sum_{i=1}^{r} \chi\left(\Delta_{i}-\bigcup_{j=1}^{I}\left\{P^{j}\right\}\right) \chi_{i} & =-32+8 n+8 l, & \sum_{j=1}^{I} \chi\left(\pi^{-1}\left(P^{j}\right)\right) & =54-9 n-9 l, \\
I^{\prime} & =6-n-l, & C=\widetilde{A}^{\prime} \cdot \widetilde{B}^{\prime}-2 l & =22 n+124-2 l .
\end{aligned}
$$

The formula for the number of cusps $C$ takes into account that each collision of $C_{0}$ with $\widetilde{\Delta}^{\prime}$ is also a cusp of $\widetilde{\Delta}^{\prime}$ which is resolved if we blow up; in fact, the collision with $\widetilde{\Delta}^{\prime}$ has multiplicity 4 and the number of cusps is reduced by 2 by each blowup. Altogether

$$
\chi(X)=-54 n-300+6 l, \quad h^{1,2}(X)=27 n+159-2 l .
$$


By [GM03, Table A] each of the $(6-n-l)$ collisions carries charged matter, contributing 27 to $n_{H}^{c h}$. One hence has $n_{H}^{c h}=162-27 n-27 l$. Anomaly cancellation according to Conjecture 4.3 holds, and comparing these data to the ones obtained for the heterotic dual (3.3.4) with $k^{1}+l=-n+12$ and $k^{2}=n+12$ according to (5.1.3), we see that Conjecture 4.2 is met.

\subsubsection{Unbroken $F_{4}$ gauge group}

If the gauge group is $H=F_{4} \times\{\mathrm{id}\}$, this means that our heterotic $K 3$ bundle has holonomy $K=G_{2} \times E_{8}$. So we are imposing $I V^{*}$ fibers on $C_{0}$ as in the previous subsection, but with nontrivial monodromy, which is the generic case. The results for $\widetilde{A}^{\prime}, \widetilde{B}^{\prime}, \widetilde{\Delta}^{\prime}, \widetilde{\Delta}^{\prime} \cdot C_{0}$ can be taken from the previous subsection, but now according to [GM03, Table 2] each intersection between $C_{0}$ and $\widetilde{\Delta}^{\prime}$ has multiplicity $\alpha_{j}=2$. These intersections are minimal, but the collisions cannot be removed by blowups. Hence we do not blow up at all, $b_{j}=0$, and

$$
\rho(Z)=2, \quad h^{1,1}(X)=7 .
$$

We have $(12-2 n)$ collisions between $C_{0}$ and $\Delta^{\prime}$. From [GM03, Table 4] the Euler characteristic of the fibers associated to such collision points is 6 . Note that in this case we have monodromy, which will affect the calculation of the charged hypermultiplets. The contributions to the formula for $\chi(X)$ in Proposition 5.1 are:

$$
\begin{aligned}
\Delta^{\prime} \cdot\left(\Delta^{\prime}+K_{\widetilde{Z}}\right) & =120 n+688, & \sum_{j} \alpha_{j}\left(\alpha_{j}-1\right) b_{j} & =0 \\
\sum_{i=1}^{r} \chi\left(\Delta_{i}-\bigcup_{j=1}^{I}\left\{P^{j}\right\}\right) \chi_{i} & =-80+16 n, & \sum_{j=1}^{I} \chi\left(\pi^{-1}\left(P^{j}\right)\right) & =72-12 n, \\
I^{\prime} & =12-2 n, & C=\widetilde{A}^{\prime} \cdot \widetilde{B}^{\prime} & =22 n+124 .
\end{aligned}
$$

Altogether

$$
\chi(X)=-48 n-336, \quad h^{1,2}(X)=24 n+175 .
$$

By [GM03, Table A] each of the $(12-2 n)$ collisions carries charged matter, with associated representation 26. However, we have monodromy, and the charged dimension of this representation is only $\operatorname{dim}(\mathbf{2 6})_{c h}=24$ according to [GM03, Table B]. Moreover, its multiplicity in $n_{H}^{c h}$ is not $(12-2 n)$ but rather $\left(g^{\prime}-g\right)=5-n$ as can be obtained from [GM03, Table E]. One hence has $n_{H}^{c h}=120-24 n$. Anomaly cancellation according to Conjecture 4.3 holds, and comparing these data to the ones obtained for the heterotic dual (3.3.5) with $k^{1}=-n+12$ and $k^{2}=n+12$ according to (5.1.3), we see that Conjecture 4.2 is met.

\subsubsection{Unbroken Spin(10) gauge group}

If the gauge group is $H=\operatorname{Spin}(10) \times\{$ id $\}$, this means that our $K 3$ bundle has holonomy $K=\mathrm{SU}(4) \times E_{8}$. So we are imposing $I_{1}^{*}$ fibers on $C_{0}$, and we have

$$
\begin{aligned}
& \widetilde{A}^{\prime}=4 L-2 C_{0}=6 C_{0}+(8+4 n) F, \\
& \widetilde{B}^{\prime}=6 L-3 C_{0}=9 C_{0}+(12+6 n) F, \\
& \widetilde{\Delta}^{\prime}=12 L-7 C_{0}=17 C_{0}+(24+12 n) F, \quad \widetilde{\Delta}^{\prime} \cdot C_{0}=24-5 n .
\end{aligned}
$$


In this case determining the multiplicities of intersection points is a bit trickier than before, because there are two different "types $P_{1}, P_{2}$ " of intersections. Using [GM03, Table E] one finds that there are $B_{1}=6-n$ intersections of "type $P_{1}$ ", and $B_{2}=4-n$ intersections of "type $P_{2}$ ". Since when counted with multiplicities the total number of intersections is $24-5 n$, one finds that intersections of "type $P_{1}$ " have multiplicity 2 , while intersections of "type $P_{2}$ " have multiplicity 3 . We choose not to blow up any of these intersections, $b_{j}=0$, yielding

$$
\rho(Z)=2, \quad h^{1,1}(X)=8 .
$$

In total, we have $B_{1}+B_{2}=10-2 n$ collisions, and from [GM03, Table 4] the Euler characteristic of the isolated fibers over collision points of both "types $P_{1}, P_{2}$ " is 8 . The contributions to the formula for $\chi(X)$ in Proposition 5.1 hence are:

$$
\begin{aligned}
& \Delta^{\prime} \cdot\left(\Delta^{\prime}+K_{\widetilde{Z}}\right)=112 n+734, \quad \quad \sum_{j} \alpha_{j}\left(\alpha_{j}-1\right) b_{j}=0, \\
& \sum_{i=1}^{r} \chi\left(\Delta_{i}-\bigcup_{j=1}^{I}\left\{P^{j}\right\}\right) \chi_{i}=-56+14 n, \quad \sum_{j=1}^{I} \chi\left(\pi^{-1}\left(P^{j}\right)\right)=80-16 n, \\
& I^{\prime}=10-2 n, \quad C=\widetilde{A}^{\prime} \cdot \widetilde{B}^{\prime}-\mu_{1} B_{1}-\mu_{2} B_{2}=20 n+136 .
\end{aligned}
$$

For the number of cusps in this case we have to apply the general formula (5.1.9) with $\mu_{1}=0$ and $\mu_{2}=2$ according to [GM03, Table 4]. Altogether

$$
\chi(X)=-52 n-312, \quad h^{1,2}(X)=26 n+164 .
$$

By [GM03, Tables A, B] each of the $(10-2 n)$ collisions carries charged matter, where the $(6-n)$ points of "type $P_{1}$ " contribute 10, and the $(4-n)$ points of "type $P_{2}$ " contribute 16, each to $n_{H}^{c h}$. One hence has $n_{H}^{c h}=124-26 n$. Anomaly cancellation according to Conjecture 4.3 holds, and comparing these data to the ones obtained for the heterotic dual (3.3.6) with $k^{1}=-n+12$ and $k^{2}=n+12$ according to (5.1.3), we see that Conjecture 4.2 is met.

\subsubsection{4 pointlike instantons on singularities of type $E_{8}, E_{7}$, or $E_{6}$}

Recall that in our Proposition 2.2 we restricted the internal $K 3$ surfaces of our heterotic string theories to be smooth, because the analysis which lead to the formulas for $n_{H}, n_{V}, n_{T}$ cannot be performed as stated if pointlike instantons collide with singularities on $K 3$. In fact, no direct technique to tackle that situation is known. However, the heterotic - F-theory duality comes to aid and allows to predict the massless spectrum even in such highly degenerate cases [AM97]. Let us describe three of the most degenerate situations, which where brought to our attention by McKay's conjecture as mentioned in the Introduction.

On the heterotic side, we assume total degeneration of the bundle data to 24 pointlike instantons. This amounts to a primordial gauge group $E_{8} \times E_{8}$ and thus to imposing $I I^{*}$ fibers on $C_{0}$ and $C_{\infty}$ in $\widetilde{Z}=\mathbb{F}_{n}$, yielding

$$
\begin{aligned}
& \widetilde{A}_{0}^{\prime}=4 L-4 C_{0}-4 C_{\infty}=8 F \\
& \widetilde{B}_{0}^{\prime}=6 L-5 C_{0}-5 C_{\infty}=2 C_{0}+(12+n) F, \\
& \widetilde{\Delta}_{0}^{\prime}=12 L-10 C_{0}-10 C_{\infty}=4 C_{0}+(24+2 n) F .
\end{aligned}
$$


Furthermore, we impose singularities of type $E_{8}, E_{7}, E_{6}$ on the heterotic $K 3$ surface. On the F-theory side, according to [AM97], this corresponds to imposing additional degenerations of the Weierstraß form over one fiber $F$ of $\widetilde{Z}=\mathbb{F}_{n}$ of type $I I^{*}, I I I^{*}, I V^{*}$, respectively. The most degenerate situation arises when all pointlike instantons coalesce on the singularity of the heterotic $K 3$ surface. Since in F-theory each pointlike instanton corresponds to a (multiple) intersection of $\widetilde{\Delta}_{0}^{\prime}$ with $C_{0}$ or $C_{\infty}$, respectively, this amounts to degenerating $\widetilde{X}$ such that all intersections of $\widetilde{\Delta}_{0}^{\prime}$ with $C_{0}$ and $C_{\infty}$ are situated also on $F$. To desingularize the highly degenerate variety $\widetilde{X}$ one performs a chain of blowups of $\widetilde{Z}$, as we shall now describe, following [AM97].

To explain the general procedure let us blow up the singularity coming from the intersection of two rational curves $\Delta_{1}, \Delta_{2}$ which carry exceptional fibers of type $\left(\alpha, \beta ; \gamma_{1}, \gamma_{2}, \gamma_{3}, \gamma_{4}, \gamma_{6}\right)$ and $\left(\alpha^{\prime}, \beta^{\prime} ; \gamma_{1}^{\prime}, \gamma_{2}^{\prime}, \gamma_{3}^{\prime}, \gamma_{4}^{\prime}, \gamma_{6}^{\prime}\right)$, respectively, such that over the intersection of $\Delta_{1}$ and $\Delta_{2}$ we have a non-minimal fiber. In other words, we assume $\alpha+\alpha^{\prime} \geq 4, \beta+\beta^{\prime} \geq 6$. We take the coordinate $t$ on $\Delta_{1}$ and $s$ on $\Delta_{2}$, such that the fibration is locally given by

$$
y^{2}+s^{\gamma_{1}} t^{\gamma_{1}^{\prime}} x y+s^{\gamma_{3}} t^{\gamma_{3}^{\prime}} y=x^{3}+s^{\gamma_{2}} t^{\gamma_{2}^{\prime}} x^{2}+s^{\gamma_{4}} t^{\gamma_{4}^{\prime}} x+s^{\gamma_{6}} t^{\gamma_{6}^{\prime}}
$$

To blow up, we set

$$
s=s_{1} t_{1}, \quad t=t_{1}, \quad x=t_{1}^{2} x_{1}, \quad y=t_{1}^{3} y_{1},
$$

i.e. we blow the base up in $s=t=0$, as well as the fiber in $x=y=0$. The equation becomes

$$
y_{1}^{2}+s_{1}^{\gamma_{1}} t_{1}^{\gamma_{1}+\gamma_{1}^{\prime}-1} x_{1} y_{1}+s_{1}^{\gamma_{3}} t_{1}^{\gamma_{3}+\gamma_{3}^{\prime}-3} y_{1}=x_{1}^{3}+s_{1}^{\gamma_{2}} t_{1}^{\gamma_{2}+\gamma_{2}^{\prime}-2} x_{1}^{2}+s_{1}^{\gamma_{4}} t_{1}^{\gamma_{4}+\gamma_{4}^{\prime}-4} x_{1}+s_{1}^{\gamma_{6}} t_{1}^{\gamma_{6}+\gamma_{6}^{\prime}-6},
$$

which along the new divisor $\left\{t_{1}=0\right\}$ has a Kodaira fiber of type

$$
\left(\alpha^{\prime \prime}, \beta^{\prime \prime} ; \gamma_{1}+\gamma_{1}^{\prime}-1, \gamma_{2}+\gamma_{2}^{\prime}-2, \gamma_{3}+\gamma_{3}^{\prime}-3, \gamma_{4}+\gamma_{4}^{\prime}-4, \gamma_{6}+\gamma_{6}^{\prime}-6\right)
$$

Additionally moving instantons into the collision point the Weierstraß model for the fibration becomes

$$
y^{2}+s^{\gamma_{1}} t^{\gamma_{1}^{\prime}} x y+s^{\gamma_{3}} t^{\gamma_{3}^{\prime}} y=x^{3}+s^{\gamma_{2}} t^{\gamma_{2}^{\prime}} x^{2}+s^{\gamma_{4}} t^{\gamma_{4}^{\prime}} x+s^{\gamma_{6}} t^{\gamma_{6}^{\prime}}\left(s+\lambda t^{k}\right)
$$

for some constant $\lambda$, and $k \in \mathbb{N}$ accounting for the number of instantons. Blowing up as before we obtain

$$
\begin{aligned}
& y_{1}^{2}+s_{1}^{\gamma_{1}} t_{1}^{\gamma_{1}+\gamma_{1}^{\prime}-1} x_{1} y_{1}+s_{1}^{\gamma_{3}} t_{1}^{\gamma_{3}+\gamma_{3}^{\prime}-3} y_{1} \\
& \quad=x_{1}^{3}+s_{1}^{\gamma_{2}} t_{1}^{\gamma_{2}+\gamma_{2}^{\prime}-2} x_{1}^{2}+s_{1}^{\gamma_{4}} t_{1}^{\gamma_{4}+\gamma_{4}^{\prime}-4} x_{1}+s_{1}^{\gamma_{6}} t_{1}^{\gamma_{6}+\gamma_{6}^{\prime}-5}\left(s_{1}+\lambda t^{k-1}\right) .
\end{aligned}
$$

We have introduced a new $\mathbb{C P}^{1}$ with fibers of type

$$
\left(\alpha^{\prime \prime}, \beta^{\prime \prime} ; \gamma_{1}+\gamma_{1}^{\prime}-1, \gamma_{2}+\gamma_{2}^{\prime}-2, \gamma_{3}+\gamma_{3}^{\prime}-3, \gamma_{4}+\gamma_{4}^{\prime}-4, \gamma_{6}+\gamma_{6}^{\prime}-5\right)
$$

\section{$E_{8}$ instantons on an $E_{8}$ singularity}

An $E_{8}$ type singularity in the $K 3$ surface on the heterotic side implies that $\widetilde{X}$ has $I I^{*}$ fibers 
not only over $C_{0}$ and $C_{\infty}$ but also over a fiber $F$. Forcing these, the divisors $\widetilde{A}_{0}^{\prime}, \widetilde{B}_{0}^{\prime}$, and $\widetilde{\Delta}_{0}^{\prime}$ become

$$
\begin{aligned}
& \widetilde{A}^{\prime}=\widetilde{A}_{0}^{\prime}-4 F=4 F \\
& \widetilde{B}^{\prime}=\widetilde{B}_{0}^{\prime}-5 F=2 C_{0}+(7+n) F \\
& \widetilde{\Delta}^{\prime}=\widetilde{\Delta}_{0}^{\prime}-10 F=4 C_{0}+(14+2 n) F .
\end{aligned}
$$

Hence

$$
\widetilde{\Delta}^{\prime} \cdot C_{0}=2(7-n), \quad \widetilde{\Delta}^{\prime} \cdot C_{\infty}=2(7+n), \quad \widetilde{\Delta}^{\prime} \cdot F=2 \cdot 2 .
$$

The first two formulas show that there are in total 14 instantons that can be moved into the $E_{8}$ singularity. There is an apparent difference between this number and the total instanton number 24 on the heterotic side; the interpretation of this mismatch in [AM97] says that the singularity on $K 3$ "eats" 10 pointlike instantons. The last formula shows that there are two further collision points of multiplicity 2 of $\widetilde{\Delta}^{\prime}$ with $F$, which will eventually be blown up.

We now move all $7 \pm n$ pointlike instantons into each collision point of two $I I_{4,5 ; 1,2,3,4,5}^{*}\left(\mathfrak{e}_{8}\right)$ fibers. This amounts to $k=7 \pm n$ in (5.3.3). Performing one blow up (5.3.5) shows that we obtain a new divisor with $(4,5 ; 1,2,3,4,5)$ type fibers, i.e. $I I_{4,5 ; 1,2,3,4,5}^{*}\left(\mathfrak{e}_{8}\right)$. According to $(5.3 .4)$, in the collision of this new divisor with $C_{0}$ or $C_{\infty}$ one still has $k-1$ pointlike instantons. Taking the two collisions of $F$ with $\widetilde{\Delta}^{\prime}$ into account, altogether we need to perform $b_{j}=14+2$ blowups of points of multiplicity $\alpha_{j}=2$ of the discriminant. Moreover, we have produced a chain of 15 $\mathbb{C P}^{1}$ 's in $Z$ with $I I^{*}$ curves in the fiber.

To smoothen the 3-fold where non-minimal fibers appear over collisions of any two curves with $I I^{*}$ fibers, another chain of blowups is necessary. Iterative application of (5.3.2) gives

$$
\begin{aligned}
& I I_{4,5 ; 1,2,3,4,5}^{*}\left(\mathfrak{e}_{8}\right)+I I_{4,5 ; 1,2,3,4,5}^{*}\left(\mathfrak{e}_{8}\right) \\
& \longrightarrow I I_{4,5 ; 1,2,3,4,5}^{*}\left(\mathfrak{e}_{8}\right)+I_{0 \mid 4,0 ; 1,2,3,4,0}+I I_{4,1 ; 1,2,3,4,1}+I V_{4,2 ; 1,2,3,4,2}(\mathfrak{s u}(2))+I_{0 \mid 4,3 ; 1,2,3,4,3}^{*}\left(\mathfrak{g}_{2}\right) \\
&+I I_{4,1 ; 1,2,3,4,1}+I V_{4,4 ; 1,2,3,4,4}^{*}\left(\mathfrak{f}_{4}\right)+I I_{4,1 ; 1,2,3,4,1}+I_{0 \mid 4,3 ; 1,2,3,4,3}^{*}\left(\mathfrak{g}_{2}\right)+I V_{4,2 ; 1,2,3,4,2}(\mathfrak{s u}(2)) \\
&+I I_{4,1 ; 1,2,3,4,1}+I_{0 \mid 4,0 ; 1,2,3,4,0}+I I_{4,5 ; 1,2,3,4,5}^{*}\left(\mathfrak{e}_{8}\right)
\end{aligned}
$$

Here we have performed 11 blowups. We have contributions to the Picard number from: the Hirzebruch surface (2); blowups from residual intersections of $\widetilde{\Delta}^{\prime}$ with $F(2)$; the chain of $\mathbb{C P}^{1}$ 's with $I I^{*}$ fibers over them (14); the additional contributions from the blowups of their collisions $(176=16 \times 11)$. In total, we get

$$
\rho(Z)=2+2+14+176=194 .
$$

John McKay remarks that $\rho(Z)=194$ is precisely the number of conjugacy classes in the Monster sporadic group $\mathbb{M}$.

As to the gauge group, each of the 15 curves in the fiber of $Z$ which carry $I I^{*}$ singularities contributes an $\mathfrak{e}_{8}$. Furthermore, by the above each of the 16 blowups of a $I I^{*}+I I^{*}$ collision contributes $\mathfrak{f}_{4} \oplus \mathfrak{g}_{2}^{\oplus 2} \oplus \mathfrak{s u}(2)^{\oplus 2}$. Together with the primordial $\mathfrak{e}_{8}^{\oplus 2}$ over $C_{0}$ and $C_{\infty}$, the total gauge algebra is

$$
\mathfrak{e}_{8}^{\oplus 17} \oplus \mathfrak{f}_{4}^{\oplus 16} \oplus \mathfrak{g}_{2}^{\oplus 32} \oplus \mathfrak{s u}(2)^{\oplus 32} .
$$


The total gauge group $H$ has dimension and rank

$$
\operatorname{dim} H=5592 \quad \text { and } \quad \operatorname{rk} H=296 .
$$

Next we need calculate $h^{1,2}(X)$. Using the algorithm described in Section 5.1 this amounts to calculating $\chi(X)$. To apply the formula given in Proposition 5.1, let us determine its various contributions:

$$
\begin{aligned}
& \widetilde{\Delta}^{\prime} \cdot\left(\widetilde{\Delta}^{\prime}+K_{\widetilde{Z}}\right)=76, \quad \sum_{j} \alpha_{j}\left(\alpha_{j}-1\right) b_{j}=2 \times 16=32, \\
& \sum_{i=1}^{r} \chi\left(\Delta_{i}-\bigcup_{j=1}^{I}\left\{P^{j}\right\}\right) \chi_{i}=20, \quad \sum_{j=1}^{I} \chi\left(\pi^{-1}\left(P^{j}\right)\right)=960 \\
& I^{\prime}=0, \quad C=\widetilde{A}^{\prime} \cdot \widetilde{B}^{\prime}=8 .
\end{aligned}
$$

From here we get

$$
\chi(X)=960, \quad h^{1,1}(X)=1+194+296=491, \quad h^{1,2}(X)=11 .
$$

Note that $h^{1,2}(X)+1=n_{H}^{0}$, the number of $K 3$ parameters on the heterotic side, where the complex structure of $K 3$ is constrained to having an $E_{8}$ singularity on $K 3$, as predicted by Conjecture 4.2. Indeed, it is conjectured that the neutral hypermultiplets are not affected by all the degenerations due to pointlike instantons coalescing with $K 3$ singularities.

Finally let us calculate $n_{H}^{c h}$. According to the explanations in Section 5.2.2 we need to consider collisions of families of degenerate curves of type (5.2.1). From the above, we have two collisions of type $I V(\mathfrak{s u}(2))+I_{0}^{*}\left(\mathfrak{g}_{2}\right)$ for each of the 16 chains obtained from blowing up $I I^{*}+I I^{*}$. This gives 32 contributions to the charged hypermultiplets. The resulting matter representation of $\mathfrak{g}_{2} \oplus \mathfrak{s u}(2)$ is $\frac{1}{2}((\mathbf{2}, \mathbf{1})+(\mathbf{2}, \mathbf{7}))$ according to [Int98], where the prefactor indicates that these representations are quaternionic and contribute with half their dimension in $n_{H}^{c h}$. Altogether we have

$$
n_{H}^{c h}=256
$$

and one checks that anomaly cancellation according to Conjecture 4.3 holds. It should be emphasized that the calculation of the charged matter representations in [Int98] uses the anomaly cancellation condition rather than giving a direct derivation. In fact, since $I_{0}^{*}\left(\mathfrak{g}_{2}\right)$ suffers monodromy, in this case not even a conjecture is known to us which describes such a direct derivation. We are in the process of filling this gap in the literature. That representations of the type exist which yield anomaly cancellation is already remarkable.

\section{$E_{8}$ instantons on an $E_{7}$ singularity}

By the same procedure as for an $E_{8}$ singularity on $K 3$, we obtain:

$$
\begin{aligned}
& \widetilde{A}^{\prime}=\widetilde{A}_{0}^{\prime}-3 F=5 F, \\
& \widetilde{B}^{\prime}=\widetilde{B}_{0}^{\prime}-5 F=2 C_{0}+(7+n) F, \\
& \widetilde{\Delta}^{\prime}=\widetilde{\Delta}_{0}^{\prime}-9 F=4 C_{0}+(15+2 n) F .
\end{aligned}
$$

Hence

$$
\widetilde{\Delta}^{\prime} \cdot C_{0}=1+2(7-n), \quad \widetilde{\Delta}^{\prime} \cdot C_{\infty}=1+2(7+n), \quad \widetilde{\Delta}^{\prime} \cdot F=2 \cdot 2 .
$$


As before, 14 instantons can be moved into the $E_{7}$ singularity. In the first two formulas the summand 1 takes into account that colliding $I I^{*}$ and $I I I^{*}$ fibers forces an additional intersection with $\widetilde{\Delta}^{\prime}$, which has multiplicity 2 on $\mathrm{F}$.

We now move all $7 \pm n$ pointlike instantons into each collision point of $I I_{4,5 ; 1,2,3,4,5}^{*}\left(\mathfrak{e}_{8}\right)$ and $I I I_{3,5 ; 1,2,3,3,5}^{*}\left(\mathfrak{e}_{7}\right)$ fibers. As before we repeatedly apply (5.3.3) with $k=7 \pm n$ obtaining 14 additional divisors with $I I I_{3,5 ; 1,2,3,3,5}^{*}\left(\mathfrak{e}_{7}\right)$ fibers. Altogether we need to perform $b_{j}=14$ blowups of points of multiplicity $\alpha_{j}=2$ of the discriminant.

The chains of blowups needed to smoothen the 3 -fold are as follows

$$
\begin{aligned}
I I_{4,5 ; 1,2,3,4,5}^{*} & \left(\mathfrak{e}_{8}\right)+I I I_{3,5 ; 1,2,3,3,5}^{*}\left(\mathfrak{e}_{7}\right) \\
\longrightarrow & I I_{4,5 ; 1,2,3,4,5}^{*}\left(\mathfrak{e}_{8}\right)+I_{0 \mid 3,0 ; 1,2,2,3,0}+I I_{3,1 ; 1,2,2,3,1}+I V_{3,2 ; 1,2,2,3,2}(\mathfrak{s u}(2))+I_{0 \mid 3,3 ; 1,2,2,3,3}^{*}\left(\mathfrak{g}_{2}\right) \\
& +I I_{2,1 ; 1,2,1,2,1}+I V_{3,4 ; 1,2,2,3,4}^{*}\left(\mathfrak{f}_{4}\right)+I I_{1,1 ; 1,2,1,1,1}+I_{0 \mid 2,3 ; 1,2,2,2,3}^{*}\left(\mathfrak{g}_{2}\right) \\
& +I I I_{1,2 ; 1,2,2,1,2}(\mathfrak{s u}(2))+I_{0 \mid 0,1 ; 1,2,2,0,1}+I I I_{3,5 ; 1,2,3,3,5}^{*}\left(\mathfrak{e}_{7}\right), \\
& I I I_{3,5 ; 1,2,3,3,5}^{*}\left(\mathfrak{e}_{7}\right)+I I I_{3,5 ; 1,2,3,3,5}^{*}\left(\mathfrak{e}_{7}\right) \\
\longrightarrow & I I I_{3,5 ; 1,2,3,3,5}^{*}\left(\mathfrak{e}_{7}\right)+I_{0 \mid 0,2 ; 1,2,3,0,2}+I I I_{1,3 ; 1,2,3,1,3}(\mathfrak{s u}(2))+I_{0 \mid 2,4 ; 1,2,3,2,4}^{*}(\mathfrak{s o}(7)) \\
& +I I I_{1,3 ; 1,2,3,1,3}(\mathfrak{s u}(2))+I_{0 \mid 0,2 ; 1,2,3,0,2}+I I I_{3,5 ; 1,2,3,3,5}^{*}\left(\mathfrak{e}_{7}\right) .
\end{aligned}
$$

Recall that the collision $I I^{*}+I I I^{*}$ forced an additional intersection with $\widetilde{\Delta}^{\prime}$. In the process of blowing up, this collision with $F$ moves onto one of the divisors carrying $I_{0}^{*}\left(\mathfrak{g}_{2}\right)$ fibers and transversally intersects it.

We have contributions to the Picard number from: the Hirzebruch surface (2); the chain of $\mathbb{C P}^{1}$ 's with $I I I^{*}$ fibers over them (14); the additional contributions from the blowups of their collisions $(90=14 \times 5+2 \times 10)$. In total, we get

$$
\rho(Z)=2+14+90=106 .
$$

The total gauge algebra is

$$
\mathfrak{e}_{8}^{\oplus 2} \oplus \mathfrak{e}_{6}^{\oplus 17} \oplus \mathfrak{s u}(2)^{\oplus 2} \oplus \mathfrak{s u}(3)^{\oplus 18} \oplus \mathfrak{g}_{2}^{\oplus 2} \oplus \mathfrak{f}_{4}^{\oplus 2} .
$$

The total gauge group $H$ has dimension and rank

$$
\operatorname{dim} H=3041 \quad \text { and } \quad \operatorname{rk} H=211 .
$$

The contributions to $\chi(X)$ in Proposition 5.1 amount to

$$
\begin{aligned}
& \widetilde{\Delta}^{\prime} \cdot\left(\widetilde{\Delta}^{\prime}+K_{\widetilde{Z}}\right)=82, \quad \sum_{j} \alpha_{j}\left(\alpha_{j}-1\right) b_{j}=2 \times 14=28, \\
& \sum_{i=1}^{r} \chi\left(\Delta_{i}-\bigcup_{j=1}^{I}\left\{P^{j}\right\}\right) \chi_{i}=8, \quad \sum_{j=1}^{I} \chi\left(\pi^{-1}\left(P^{j}\right)\right)=630, \\
& I^{\prime}=2, \quad C=\widetilde{A}^{\prime} \cdot \widetilde{B}^{\prime}=10 .
\end{aligned}
$$

From here we get

$$
\chi(X)=612, \quad h^{1,1}(X)=318, \quad h^{1,2}(X)=12 .
$$


Again $h^{1,2}(X)+1=n_{H}^{0}$, the number of $K 3$ parameters on the heterotic side, where the complex structure of $K 3$ is constrained to having an $E_{7}$ singularity on $K 3$, as predicted by Conjecture 4.2 .

For $n_{H}^{c h}$ we list collisions of curves of type (5.2.1): We have 4 collisions $I V(\mathfrak{s u}(2))+I_{0}^{*}\left(\mathfrak{g}_{2}\right)$ and $I I I(\mathfrak{s u}(2))+I_{0}^{*}\left(\mathfrak{g}_{2}\right)$ each as well as $2 \times 14$ collisions $I I I(\mathfrak{s u}(2))+I_{0}^{*}(\mathfrak{s o}(7))$. According to [Int98], the associated representations are, respectively, $\frac{1}{2}((\mathbf{2}, \mathbf{1})+(\mathbf{2}, \mathbf{7}))$ of $\mathfrak{s u}(2) \oplus \mathfrak{g}_{2}$ and $\frac{1}{2}(\mathbf{2}, \mathbf{8})$ of $\mathfrak{s u}(2) \oplus \mathfrak{s o}(7)$ amounting to a total of

$$
n_{H}^{c h}=256 .
$$

The same comment as above applies to the derivation of the charged representations in [Int98]. One checks that anomaly cancellation according to Conjecture 4.3 holds. We do not yet understand why the remaining collisions of $\Delta^{\prime}$ with families of $I_{0}^{*}\left(\mathfrak{g}_{2}\right)$ fibers do not contribute to the charged hypermultiplets.

\section{$E_{8}$ instantons on an $E_{6}$ singularity}

This time we have

$$
\begin{aligned}
\widetilde{A}^{\prime} & =\widetilde{A}_{0}^{\prime}-3 F=4 F \\
\widetilde{B}^{\prime} & =\widetilde{B}_{0}^{\prime}-4 F=2 C_{0}+(8+n) F, \\
\widetilde{\Delta}^{\prime} & =\widetilde{\Delta}_{0}^{\prime}-8 F=4 C_{0}+(16+2 n) F \\
\widetilde{\Delta}^{\prime} \cdot C_{0} & =2(8-n), \quad \widetilde{\Delta}^{\prime} \cdot C_{\infty}=2(8+n), \quad \widetilde{\Delta}^{\prime} \cdot F=4 .
\end{aligned}
$$

Hence 16 instantons can be moved into the $E_{6}$ singularity. In order to consistently impose families of $I I^{*}$ and $I V^{*}$ fibers like this, the $I V^{*}$ fibers cannot suffer from monodromy. The collision then forces an additional intersection with $\widetilde{\Delta}^{\prime}$ in the intersection $I I^{*}+I V^{*}$ with multiplicity 2. This is accounted for in the last formula above and also contributes 2 to each of the intersections of $\widetilde{\Delta}^{\prime}$ with $C_{0}$ and $C_{\infty}$.

We now move all $7 \pm n$ free pointlike instantons into each collision point of $I I_{4,5 ; 1,2,3,4,5}^{*}\left(\mathfrak{e}_{8}\right)$ and $I V_{3,4 ; 1,2,2,3,5}^{*}\left(\mathfrak{e}_{6}\right)$ fibers. As before we repeatedly apply (5.3.3) with $k=7 \pm n$. Altogether 14 blowups introduce additional divisors with $I V_{3,4 ; 1,2,2,3,5}^{*}\left(\mathfrak{e}_{6}\right)$ fibers. In this situation, the collisions $I I^{*}+I V^{*}$ still force an additional intersection with $\widetilde{\Delta}^{\prime}$, which make one further blowup of the discriminant necessary each, yielding new divisors with $I V_{3,4 ; 1,2,2,3,4}^{*}\left(\mathfrak{f}_{4}\right)$ fibers over them. The latter intersect $\widetilde{\Delta}^{\prime}$ with multiplicity 2 away from other collisions. Altogether we perform $b_{j}=16$ blowups of points of multiplicity $\alpha_{j}=2$ of the discriminant.

The chains of blowups needed to smoothen the 3 -fold are as follows

$$
\begin{aligned}
& I I_{4,5 ; 1,2,3,4,5}^{*}\left(\mathfrak{e}_{8}\right)+I V_{3,4 ; 1,2,2,3,4}^{*}\left(\mathfrak{f}_{4}\right) \\
& \quad I I_{4,5 ; 1,2,3,4,5}^{*}\left(\mathfrak{e}_{8}\right)+I_{0 \mid 3,0 ; 1,2,2,3,0}+I I_{3,1 ; 1,2,2,3,1}+I V_{3,2 ; 1,2,2,3,2}(\mathfrak{s u}(2)) \\
& \quad+I_{0 \mid 3,3 ; 1,2,2,3,3}^{*}\left(\mathfrak{g}_{2}\right)+I I_{2,1 ; 1,2,1,2,1}+I V_{3,4 ; 1,2,2,3,4}^{*}\left(\mathfrak{f}_{4}\right), \\
& I V_{3,4 ; 1,2,2,3,4}^{*}\left(\mathfrak{f}_{4}\right)+I V_{3,4 ; 1,2,2,3,5}^{*}\left(\mathfrak{e}_{6}\right) \\
& \quad I V_{3,4 ; 1,2,2,3,4}^{*}\left(\mathfrak{f}_{4}\right)+I_{0 \mid 1,0 ; 1,2,0,1,1}+I V_{2,2 ; 1,2,1,2,3}(\mathfrak{s u}(3))+I_{0 \mid 1,0 ; 1,2,0,1,2}+I V_{3,4 ; 1,2,2,3,5}^{*}\left(\mathfrak{e}_{6}\right), \\
& I V_{3,4 ; 1,2,2,3,5}^{*}\left(\mathfrak{e}_{6}\right)+I V_{3,4 ; 1,2,2,3,5}^{*}\left(\mathfrak{e}_{6}\right) \\
& \quad I V_{3,4 ; 1,2,2,3,5}^{*}\left(\mathfrak{e}_{6}\right)+I_{0 \mid 1,0 ; 1,2,0,1,3}+I V_{2,2 ; 1,2,1,2,4}(\mathfrak{s u}(3))+I_{0 \mid 1,0 ; 1,2,0,1,3}+I V_{3,4 ; 1,2,2,3,5}^{*}\left(\mathfrak{e}_{6}\right) .
\end{aligned}
$$


We have contributions to the Picard number from: the Hirzebruch surface (2); the chain of $\mathbb{C P}^{1}$ 's with $I V^{*}$ fibers over them (16); the additional contributions from the blowups of their collisions $(58=2 \times 5+16 \times 3)$. In total, we get

$$
\rho(Z)=2+16+58=76 .
$$

The total gauge algebra is

$$
\mathfrak{e}_{8}^{\oplus 2} \oplus \mathfrak{e}_{6}^{\oplus 15} \oplus \mathfrak{f}_{4}^{\oplus 2} \oplus \mathfrak{s u}(2)^{\oplus 2} \oplus \mathfrak{g}_{2}^{\oplus 2} \oplus \mathfrak{s u}(3)^{\oplus 16} .
$$

The total gauge group $H$ has dimension and rank

$$
\operatorname{dim} H=1932 \text { and } \quad \operatorname{rk} H=152 .
$$

The contributions to $\chi(X)$ in Proposition 5.1 amount to

$$
\begin{aligned}
& \widetilde{\Delta}^{\prime} \cdot\left(\widetilde{\Delta}^{\prime}+K_{\widetilde{Z}}\right)=88, \quad \sum_{j} \alpha_{j}\left(\alpha_{j}-1\right) b_{j}=2 \times 16=32, \\
& \sum_{i=1}^{r} \chi\left(\Delta_{i}-\bigcup_{j=1}^{I}\left\{P^{j}\right\}\right) \chi_{i}=4, \quad \sum_{j=1}^{I} \chi\left(\pi^{-1}\left(P^{j}\right)\right)=456, \\
& I^{\prime}=2, \quad C=\widetilde{A}^{\prime} \cdot \widetilde{B}^{\prime}=10 .
\end{aligned}
$$

From here we get

$$
\chi(X)=432, \quad h^{1,1}(X)=229, \quad h^{1,2}(X)=13 .
$$

Once again $h^{1,2}(X)+1=n_{H}^{0}$, the number of $K 3$ parameters on the heterotic side, where the complex structure of $K 3$ is constrained to having an $E_{6}$ singularity on $K 3$, as predicted by Conjecture 4.2.

For $n_{H}^{c h}$ we list collisions of curves of type (5.2.1): We have 2 collisions $I V(\mathfrak{s u}(2))+I_{0}^{*}\left(\mathfrak{g}_{2}\right)$ which according to [Int98] have associated representations $\frac{1}{2}((\mathbf{2}, \mathbf{1})+(\mathbf{2}, \mathbf{7}))$ of $\mathfrak{s u}(2) \oplus \mathfrak{g}_{2}$ amounting to a total

$$
n_{H}^{c h}=16 .
$$

One checks that anomaly cancellation according to Conjecture 4.3 holds. Again we do not understand why the remaining collisions of $\widetilde{\Delta}^{\prime}$ with families of $I V^{*}\left(\mathfrak{f}_{4}\right)$ fibers do not contribute to the charged hypermultiplets.

\section{Conclusions}

Having devoted the bulk of this work to the description of our understanding of aspects of the heterotic - F-theory duality, we would like to return to the original motivation of this project, namely a new conjecture by John McKay. His conjecture relates geometric data of Calabi-Yau three-folds on the F-theory side of this duality to the Monster sporadic group and its offspring. Namely, as before let $X \rightarrow Z$ denote the Calabi-Yau three-fold which arises as F-theory dual of the heterotic theory with 24 pointlike $E_{8}$ instantons localized at an $E_{8}$ type quotient singularity on K3. Then following [AM97, AKM00] for the Picard number of the base we have shown 
$\rho(Z)=194$, which as John McKay has observed agrees with the number of conjugacy classes of the Monster sporadic group $\mathbb{M}$. He conjectures that this is not a coincidence.

As described in the Introduction, McKay supports his conjecture by a known relation between the conjugacy classes of $\mathbb{M}$ and the Dynkin data of $E_{8}$ [McK80, GN01]. Also note that 24 pointlike instantons in an $E_{8}$ type quotient singularity give the most degenerate case of the heterotic - F-theory duality with the maximal number of pointlike instantons in the worst possible singularity on $K 3$. Relating the F-theory dual Calabi-Yau three-fold $X \rightarrow Z$ to the largest finite sporadic group may not be completely unexpected, in particular as the Euler characteristic of this three-fold is 960, see Section 5.3.7, the largest value among all known elliptically fibered Calabi-Yau three-folds.

Naturally one would like to support McKay's conjecture by further data points. A possible lead is the above-mentioned relation between $\mathbb{M}$ and the Dynkin data of $E_{8}$, which roughly extends to relating the Baby monster $\mathbb{B}$ to $E_{7}$ and the Fischer group $\mathrm{Fi}_{24}$ to $E_{6}$ [GN01]. However, see Section 5.3.7, the respective Picard numbers $\rho(Z)$ of the bases of the F-theory dual Calabi-Yau three-folds corresponding to 24 pointlike $E_{8}$ instantons in quotient singularities of type $E_{7}$ and $E_{6}$ do not agree with the numbers of conjugacy classes of $\mathbb{B}$ and $\mathrm{Fi}_{24}$. Hence McKay's conjecture requires some refinement. This may be related to the details of the identification of group data and Dynkin data for $\mathbb{B} \leftrightarrow \widetilde{E}_{7}$ and $\mathrm{Fi}_{24} \leftrightarrow \widetilde{\mathrm{E}}_{6}$. As explained in the Introduction, the relevant Dynkin data are obtained via folding $\widetilde{E}_{7}$ and $\widetilde{E}_{6}$ to the non-simply laced diagrams $\widetilde{F}_{4}$ and $\widetilde{G}_{2}$. Therefore one would like to find a way of implementing this folding procedure geometrically on the F-theory side. One promising possibility could amount to making use of less standard orbifold techniques, like Slodowy's interpretation of non-simply laced Dynkin diagrams in the description of the geometry of certain quotient singularities [Slo80].

In conclusion, at this point McKay's new conjecture is definitely not settled. However, it directs towards innovative and beautiful mathematics. Even if the foundation of the conjecture ${ }^{5}$ is rather weak, we hope that by now the reader appreciates the importance and depth of the duality and its geometric meaning, be the conjecture true or wrong.

I have that sneaking hope, a hope unsupported by any facts or any evidence, that sometime in the twenty-first century physicists will stumble upon the Monster group, built in some unsuspected way into the structure of the universe.

(F.J. Dyson, "Unfashionable Pursuits", Math. Intelligencer 5 (1983), no. 3, 47-54)

\section{A Rarita-Schwinger fields}

In the physics literature, massless fields are called Rarita-Schwinger fields, if they transform in the highest irreducible component RS of $S \otimes V$ where $S$ denotes the spinor representation, and $V$ denotes the vector representation of $\mathfrak{s o}(D-2)$. However, this terminology is not used completely consistently and great care has to be taken with it: For example, let $M=M^{1, D-1}$ and denote by $S_{M}^{ \pm}$the corresponding spinor bundles. Naively, a massless Rarita-Schwinger field is a section in $S_{M}^{+} \otimes T^{*} M$ which vanishes under the associated Dirac operator $\not D$. However, reduction to

\footnotetext{
${ }^{5} E_{8}$ vs. 194
} 
$\mathfrak{s o}(D-2)$ yields $T_{x}^{*} M \cong V \oplus \mathbf{1} \oplus \mathbf{1}$ for every $x \in M$, and moreover $S^{+} \otimes V=S^{-} \oplus \mathrm{RS}^{+}$, where now $S^{ \pm}$are the components of the spin representation of $\mathfrak{s o}(1, D-1)$ arising under reduction to $\mathfrak{s o}(D-2)$. Hence to extract $\mathrm{RS}^{+}$we need to work on a virtual bundle and with the Dirac operator

$$
\not D_{R S}:\left(S^{+} \otimes T^{*} M\right) \ominus S^{+} \ominus S^{+} \ominus S^{-} \longrightarrow\left(S^{-} \otimes T^{*} M\right) \ominus S^{-} \ominus S^{-} \ominus S^{+} .
$$

By stretching of terminology, even if $M$ is Euclidean one calls the above the Rarita-Schwinger complex of $M$, where $S^{ \pm}$denote the spinor bundles on $M$ [ASZ84, §IV.V].

Remark. For $D=4$ the bundles $S^{ \pm}$have dimension two each, such that $S^{+} \otimes S^{-} \cong T^{*} M$, and if $\Lambda^{2} S^{+} \cong \mathbf{1}$ then $S^{+} \otimes T^{*} M \ominus S^{+} \ominus S^{+} \ominus S^{-} \cong \operatorname{Sym}^{2}\left(\mathrm{~S}^{+}\right) \otimes \mathrm{S}^{-} \ominus\left(\mathrm{S}^{+} \oplus \mathrm{S}^{+}\right)$, where $\mathrm{Sym}^{2}$ denotes the two-symmetric tensor product. This latter virtual bundle is given as the standard domain of Rarita-Schwinger operators in [EGH80] if $M$ is a $K 3$ surface.

To compute the index of $\not_{R S}$, one introduces the Dirac operator $\widetilde{D}_{R S}$ on $S^{+} \otimes T^{*} M$ and uses the additivity of the index to get

$$
\operatorname{ind}\left(\not D_{R S}\right)=\operatorname{ind}\left(\widetilde{D}_{R S}\right)-\operatorname{ind}(\not D)
$$

where $\not D: S^{+} \rightarrow S^{-}$.

Note that the definition of the index requires us to work with complex vector bundles, so for real fields we simply complexify real representations.

\section{B Characteristic classes and properties of $K 3$ surfaces}

We collect a few properties of characteristic classes in particular for $K 3$, carefully keeping track of all the prefactors.

Let $\mathcal{E}$ be a complex bundle over a manifold $X$, with connection $A$ (which is a $\mathfrak{u}(n)$ valued 1-form) and associated curvature $F_{\mathcal{E}}$. To it one associates the total Chern form:

$$
c\left(F_{\mathcal{E}}\right)=\operatorname{det}\left(I_{n}+\frac{i}{2 \pi} F_{\mathcal{E}}\right)
$$

where $I_{n}$ is the $n \times n$ identity matrix. The integral of the component in each degree gives the corresponding Chern class of $\mathcal{E}$ :

$$
c_{1}(\mathcal{E})=\frac{i}{2 \pi} \int_{X} \operatorname{Trace}\left(F_{\mathcal{E}}\right), \quad c_{2}(\mathcal{E})=\frac{1}{8 \pi^{2}} \int_{X}\left(\operatorname{Trace}\left(F_{\mathcal{E}}^{2}\right)-\left(\operatorname{Trace}\left(F_{\mathcal{E}}\right)\right)^{2}\right), \quad \ldots
$$

We also have the Chern character

$$
\operatorname{ch}(\mathcal{E})=\int_{X} \text { Trace }\left[\exp \left(\frac{i}{2 \pi} F_{\mathcal{E}}\right)\right]=d+c_{1}(\mathcal{E})+\frac{1}{2}\left(c_{1}^{2}(\mathcal{E})-2 c_{2}(\mathcal{E})\right)+\ldots,
$$

where $d$ is the rank of the complex bundle $\mathcal{E}$. 
Now for a real vector bundle $E$, with connection 1 -form valued in $\mathfrak{s o}(n)$ this time, and with curvature $F_{E}$, the corresponding form is the total Pontrjagin form

$$
p\left(F_{E}\right)=\operatorname{det}\left(I_{n}+\frac{1}{2 \pi} F_{E}\right)
$$

which gives the Pontrjagin classes of $E$ when the component in each degree is integrated over $X$. Note that $F_{E}^{T}=-F_{E}$ such that only the even powers of $F_{E}$ contribute. One sets

$$
p_{k}(E):=\int_{X} p\left(F_{E}\right)_{4 k} \quad \text { such that } \quad p_{1}(E)=-\frac{1}{8 \pi^{2}} \int_{X} \operatorname{Trace}\left(F_{E}^{2}\right), \quad \ldots
$$

We can analyze the Pontrjagin classes in terms of Chern classes for a real vector bundle $E$,

$$
p_{k}(E)=(-1)^{k} c_{2 k}(E \otimes \mathbb{C}) .
$$

Conversely, if we are given a complex vector bundle $\mathcal{E}$, we introduce a real vector bundle $E$ such that $\mathcal{E} \oplus \overline{\mathcal{E}}=\mathbb{C} \otimes E$ and therefore

$$
p_{1}(E)=\left(c_{1}^{2}-2 c_{2}\right)(\mathcal{E})=-\frac{1}{4 \pi^{2}} \int_{X} \operatorname{Trace}\left(F_{\mathcal{E}}^{2}\right)=-\frac{1}{8 \pi^{2}} \int_{X} \operatorname{Trace}\left(F_{E}^{2}\right),
$$

since a diagonal matrix in $\mathfrak{u}(n)$ with the entry $i x_{j}$ is mapped to $\left[\begin{array}{ll}0 & -x_{j} \\ x_{j} & 0\end{array}\right]$ in $\mathfrak{s o}(2 n)$. This also gives a cross-check for the formula for the Pontrjagin class.

On a spin manifold $X$ with Riemannian curvature $R$, we have the $A$-hat form

$$
\widehat{A}(R)=1-\frac{1}{24} p_{1}(R)+\frac{1}{5760}\left(7 p_{1}^{2}-4 p_{2}\right)+\ldots,
$$

which when integrated over $X$ gives the index of the Dirac operator $\not D: S^{+} \rightarrow S^{-}$,

$$
\text { ind }(\not D)=\int_{X} \widehat{A}(R) \text {. }
$$

For a twisted Dirac operator $\not D_{\mathcal{E}}$, with $S^{+}$twisted by a complex vector bundle $\mathcal{E}$ of rank $d$, we have $\not D_{\mathcal{E}}: S^{+} \otimes \mathcal{E} \longrightarrow S^{-} \otimes \mathcal{E}$ and

$$
\text { ind }\left(\not D_{\mathcal{E}}\right)=\int_{X} \widehat{A}(R) \operatorname{ch}(\mathcal{E})
$$

If we assume that $X$ is a 4-manifold then the above considerations give

$$
\text { ind }(\not D)=-\frac{1}{24} \int_{X} p_{1}(R)=\frac{1}{24 \cdot 8 \pi^{2}} \int_{X} \operatorname{Trace}\left(R^{2}\right) \text {, }
$$

and its twisted version

$$
\begin{aligned}
\text { ind }\left(\not D_{\mathcal{E}}\right) & =-\frac{d}{24} \int_{X} p_{1}(R)+\frac{1}{2} \int_{X}\left(c_{1}^{2}\left(F_{\mathcal{E}}\right)-2 c_{2}\left(F_{\mathcal{E}}\right)\right) \\
& =\frac{d}{24 \cdot 8 \pi^{2}} \int_{X} \operatorname{Trace}\left(R^{2}\right)-\frac{1}{8 \pi^{2}} \int_{X} \operatorname{Trace}\left(F_{\mathcal{E}}^{2}\right) .
\end{aligned}
$$


Now for a $K 3$ surface we have the signature

$$
\tau(K 3)=\frac{1}{3} \int_{K 3} p_{1}(R)=-16,
$$

the Euler characteristic

$$
\chi(K 3)=\int_{K 3} c_{2}(R)=24,
$$

and the $A$-hat genus

$$
\widehat{A}(K 3)=-\frac{1}{8} \tau=2 .
$$

Also, on a spin four-manifold $X$ the index of the twisted Dirac operator $\widetilde{D}_{R S}$ on $S^{+} \otimes T^{*} X$ is

$$
\text { ind }\left(\widetilde{D}_{R S}\right)(X)=\frac{20}{24} \int_{X} p_{1}(R) \text {. }
$$

By (A.7) the Rarita-Schwinger operator then has index

$$
\text { ind }\left(\not D_{R S}\right)(X)=\frac{21}{24} \int_{X} p_{1}(R) \text {. }
$$

In particular, for a $K 3$ surface $X$ we obtain

$$
\text { ind }\left(\widetilde{D}_{R S}\right)(X)=-40 \text {. }
$$

\section{References}

[Adl] S.L. ADLER, Anomalies; hep-th/0411038.

[Adl69] — Axial vector vertex in spinor electrodynamics, Phys. Rev. 177 (1969), 24262438.

[AG96] P.S. Aspinwall and M. Gross, The SO(32) Heterotic String on a K3 Surface, Phys. Lett. B387 (1996), 735-742; hep-th/9605131.

[AGG85] L. Alvarez-Gaumé And P.H. Ginsparg, The structure of gauge and gravitational anomalies, Ann. Physics 161 (1985), 423-526, Erratum-ibid.171:233,1986.

[AGW84] L. Alvarez-Gaumé And E. Witten, Gravitational anomalies, Nucl. Phys. B234 (1984), 269-379.

[AHS78] M.F. Atiyah, N.J. Hitchin, And I.M. Singer, Self-duality in four-dimensional Riemannian geometry, prsla 362 (1978), no. 1711, 425-461.

[AKM00] P.S. Aspinwall, S. Katz, And D.R. MorRison, Lie groups, Calabi-Yau threefolds, and F-theory, Adv. Theor. Math. Phys. 4 (2000), 95-126; hep-th/0002012. 
[AL96] P.S. Aspinwall And J. Louis, On the Ubiquity of K3 Fibrations in String Duality, Phys. Lett. B369 (1996), 233-242; hep-th/9510234.

[AM94] P.S. Aspinwall And D.R. Morrison, String theory on K3 surfaces, in: Mirror symmetry II, B. Greene and S.T. Yau, eds., 1994, pp. 703-716; hep-th/9404151.

[AM97] —, Point-like instantons on K3 orbifolds, Nucl. Phys. B503 (1997), 533-564; hep-th/9705104.

[AS71] M.F. Atiyah And I.M. Singer, The index of elliptic operators IV, Adv. Math. 93 (1971), 119-138.

[AS84] - Dirac operators coupled to vector potentials, Proc. Nat. Acad. Sci. U.S.A. 81 (1984), no. 8, Phys. Sci., 2597-2600.

[Asp97] P.S. Aspinwall, K3 surfaces and string duality, in: Fields, strings and duality (Boulder, CO, 1996), World Sci. Publishing, River Edge, NJ, 1997, pp. 421-540; hep-th/9611137.

[ASZ84] O. Alvarez, I.M. Singer, And B. Zumino, Gravitational anomalies and the family's index theorem, Commun. Math. Phys. 96 (1984), no. 3, 409-417.

[Ati84] M. AtiYah, Anomalies and index theory, in: Supersymmetry and supergravity/nonperturbative QCD (Mahabaleshwar, 1984), vol. 208 of Lecture Notes in Phys., Springer, Berlin, 1984, pp. 313-322.

[Bar69] W.A. Bardeen, Anomalous Ward identities in spinor field theories, Phys. Rev. 184 (1969), 1848-1857.

[BCG $\left.{ }^{+} 98\right]$ M. Bershadsky, T.M. Chiang, B.R. Greene, A. Johansen, and C.I. Lazaroiu, F-theory and linear sigma models, Nucl. Phys. B527 (1998), 531-570; hep-th/9712023.

[BIK ${ }^{+}$96] M. Bershadsky, K. Intriligator, S. Kachru, D.R. Morrison, V. Sadov, AND C. VAFA, Geometric singularities and enhanced gauge symmetries, Nucl. Phys. B481 (1996), 215-252; hep-th/9605200.

[BJPS97] M. Bershadsky, A. Johansen, T. Pantev, and V. Sadov, On fourdimensional compactifications of F-theory, Nucl. Phys. B505 (1997), 165-201; hep-th/9701165.

[BM03] A. Bilal And S. Metzger, Anomaly cancellation in M-theory: A critical review, Nucl. Phys. B675 (2003), 416-446; hep-th/0307152.

[Bor01] R.E. Borcherds, Problems in Moonshine, in: First International Congress of Chinese Mathematicians (Beijing, 1998), vol. 20 of AMS/IP Stud. Adv. Math., Amer. Math. Soc., Providence, RI, 2001, pp. 3-10.

[Bor02] R. E. Borcherds, What is the monster?, Notices of the A.M.S 49 (2002), no. 9, 1076-1077; arXiv:math.GR/0209328. 
[BZ84] W.A. BARdeen And B. Zumino, Consistent and covariant anomalies in gauge and gravitational theories, Nucl. Phys. B244 (1984), 421.

[CPR98] P. Candelas, E. Perevalov, and G. Rajesh, Matter from toric geometry, Nucl. Phys. B519 (1998), 225-238; hep-th/9707049.

[DE99] D.-E. Diaconescu And R. Entin, Calabi-Yau spaces and five-dimensional field theories with exceptional gauge symmetry, Nucl. Phys. B538 (1999), 451-484; hep-th/9807170.

[DHVW85] L.J. Dixon, J. Harvey, C. Vafa, and E. Witten, Strings on orbifolds, Nucl. Phys. B261 (1985), 678-686.

[DHVW86] — Strings on orbifolds II, Nucl. Phys. B274 (1986), 285-314.

[DK90] S.K. Donaldson And P.B. Kronheimer, The geometry of four-manifolds, Oxford Mathematical Monographs, Oxford University Press, New York, 1990.

[DMW96] M.J. Duff, R. Minasian, And E. Witten, Evidence for Heterotic/Heterotic Duality, Nucl. Phys. B465 (1996), 413-438; hep-th/9601036.

[Don85] S.K. Donaldson, Anti-self-dual Yang-Mills connections on complex algebraic surfaces and stable vector bundles, Proc. Lond. Math. Soc. 3 (1985), 1-26.

[EGH80] T. Eguchi, P.B. Gilkey, And A.J. Hanson, Gravitation, gauge theories and differential geometry, Phys. Rev. 66 (1980), 213.

[FHSV95] S. Ferrara, J.A. Harvey, A. Strominger, and C. Vafa, Second quantized mirror symmetry, Phys. Lett. B361 (1995), 59-65; hep-th/9505162.

[FMW97] R. Friedman, J. Morgan, And E. Witten, Vector bundles and F theory, Commun. Math. Phys. 187 (1997), no. 3, 679-743; hep-th/9701162.

[GH78] P. Griffiths And J. Harris, Principles of algebraic geometry, John Wiley \& Sons, New York, 1978.

[GM03] A. Grassi And D.R. MorRISOn, Group representations and the Euler characteristic of elliptically fibered Calabi-Yau threefolds, J. Alg. Geom. 12 (2003), 321-356; math.ag/0005196.

[GN01] G. Glauberman And S.P. Norton, On McKay's connection between the affine $E_{8}$ diagram and the Monster, Proceedings on Moonshine and related topics (Montréal, QC, 1999) (Providence, RI), CRM Proc. Lecture Notes, vol. 30, Amer. Math. Soc., pp. $37-42$.

[GS84] M.B. Green And J.H. Schwarz, Anomaly cancellation in supersymmetric D = 10 gauge theory and superstring theory, Phys. Lett. B149 (1984), 117-122.

[GS85a] —, The hexagon gauge anomaly in type I superstring theory, Nucl. Phys. B255 (1985), no. 1, 93-114. 
[GS85b] - Infinity cancellations in SO(32) superstring theory, Phys. Lett. B151 (1985), no. $1,21-25$.

[GSW85] M.B. Green, J.H. Schwarz, and P.C. West, Anomaly free chiral theories in six dimensions, Nucl. Phys. B254 (1985), 327-348.

[GSW87] M. Green, J.H. Schwarz, And E. Witten, Superstring theory I 8 II, Cambridge University Press, 1987.

[Har05] J.A. HARVEY, TASI 2003 lectures on anomalies; hep-th/0509097.

[HT95] C.M. Hull And P.K. Townsend, Unity of superstring dualities, Nucl. Phys. B438 (1995), 109-137; hep-th/9410167.

[IMS97] K.A. Intriligator, D.R. Morrison, And N. Seiberg, Five-dimensional supersymmetric gauge theories and degenerations of Calabi-Yau spaces, Nucl. Phys. B497 (1997), 56-100; hep-th/9702198.

[Int98] K.A. Intriligator, New string theories in six dimensions via branes at orbifold singularities, Adv. Theor. Math. Phys. 1 (1998), 271-282; hep-th/9708117.

[KMP96] S. Katz, D.R. Morrison, and M.R. Plesser, Enhanced Gauge Symmetry in Type II String Theory, Nucl. Phys. B477 (1996), 105-140; hep-th/9601108.

[Kod64] K. KodAIRA, On the structure of compact complex analytic surfaces, I. Am. J. Math. 86 (1964), 751-798.

[KV95] S. KachrU AND C. VAFA, Exact results for $N=2$ compactifications of heterotic strings, Nucl. Phys. B450 (1995), 69-89; hep-th/9505105.

[KV97] S. Katz And C. VAFA, Matter from geometry, Nucl. Phys. B497 (1997), 146-154; hep-th/9606086.

[McK80] J. McKay, Graphs, singularities, and finite groups, in: The Santa Cruz Conference on Finite Groups (Univ. California, Santa Cruz, Calif., 1979), Amer. Math. Soc., Providence, R.I., 1980, pp. 183-186.

[Mir83] R. Miranda, Smooth models for elliptic threefolds, in: The birational geometry of degenerations (Cambridge, Mass., 1981), vol. 29 of Progr. Math., Birkhäuser Boston, Mass., 1983, pp. 85-133.

[MP81] W.G. MCKAY AND J. PaterA, Tables of dimensions, indices, and branching rules for representations of simple Lie algebras, Lecture Notes in Pure and Applied Mathematics, vol. 69, Marcel Dekker Inc., New York, 1981.

[MV96a] D.R. Morrison And C. VAFA, Compactifications of F-Theory on Calabi-Yau Threefolds - I, Nucl. Phys. B473 (1996), 74-92; hep-th/9602114.

[MV96b] —, Compactifications of F-Theory on Calabi-Yau Threefolds - II, Nucl. Phys. B476 (1996), 437-469; hep-th/9603161. 
[Pol98] J. Polchinski, String theory. Vols. I \& II, Cambridge Monographs on Mathematical Physics, Cambridge University Press, Cambridge, 1998.

[PS95] M.E. PEskin AND D.V. Schroeder, An introduction to quantum field theory, Addison-Wesley Publishing Company Advanced Book Program, Reading, MA, 1995, Edited and with a foreword by David Pines.

[Sad96] V. Sadov, Generalized Green-Schwarz mechanism in F theory, Phys. Lett. B388 (1996), 45-50; hep-th/9606008.

[Sch96] J.H. Schwarz, Anomaly-Free Supersymmetric Models in Six Dimensions, Phys. Lett. B371 (1996), 223-230; hep-th/9512053.

[Sch02] —, Anomaly cancellation: A retrospective from a modern perspective, Int. J. Mod. Phys. A17S1 (2002), 157-166; hep-th/0107059.

[Sei88] N. Seiberg, Observations on the moduli space of superconformal field theories, Nucl. Phys. B303 (1988), 286-304.

[Sen96a] A. Sen, F-theory and Orientifolds, Nucl. Phys. B475 (1996), 562-578; hep-th/9605150.

[Sen96b] —, M-Theory on $\left(K 3 \times S_{1}\right) / Z_{2}$, Phys. Rev. D53 (1996), 6725-6729; hep-th/9602010.

[Slo80] P. Slodowy, Simple singularities and simple algebraic groups, Lecture Notes in Mathematics, vol. 815, Springer, Berlin, 1980.

[SS04] C.A. Scrucca And M. Serone, Anomalies in field theories with extra dimensions, Int. J. Mod. Phys. A19 (2004), 2579-2642; hep-th/0403163.

[Stu03] E. Study, Geometrie der Dynamen, Leipzig: Teubner, 1903.

[SW96] N. Seiberg And E. Witten, Comments on String Dynamics in Six Dimensions, Nucl. Phys. B471 (1996), 121-134; hep-th/9603003.

[UY86] K.K. Uhlenbeck And S.-T. YAU, On the existence of Hermitian-Yang-Mills connections in stable vector bundles, Commun. Pure Appl. Math. Suppl. 39(S) (1986), $257-293$.

[Vaf96] C. VAFA, Evidence for F-Theory, Nucl. Phys. B469 (1996), 403-418; hep-th/9602022.

[Wal88] M. Walton, The Heterotic string on the simplest Calabi-Yau manifold and its orbifold limits, Phys. Rev. D 37 (1988), 377-390.

[Wei05] S. Weinberg, The quantum theory of fields. Vol. II, Cambridge University Press, Cambridge, 2005, Modern applications. 
[Wit95] E. Witten, String theory dynamics in various dimensions, Nucl. Phys. B443 (1995), 85-126; hep-th/9503124.

[Wit96a] _ Phase Transitions In M-Theory And F-Theory, Nucl. Phys. B471 (1996), 195$216 ;$ hep-th/9603150.

[Wit96b] — Physical interpretation of certain strong coupling singularities, Mod. Phys. Lett. A11 (1996), 2649-2654; hep-th/9609159.

[Wit96c] —, Strong Coupling Expansion Of Calabi-Yau Compactification, Nucl. Phys. B471 (1996), 135-158; hep-th/9602070.

[WZ71] J. Wess And B. Zumino, Consequences of anomalous Ward identities, Phys. Lett. B37 (1971), 95.

Anda Degeratu

Dept. of Mathematics

Duke University

Durham NC 27708, USA

email: anda@math.duke.edu
Katrin Wendland

Dept. of Mathematics

UNC Chapel Hill

Chapel Hill, NC 25599, USA;

and

Mathematics Institute

University of Warwick

CV4-7AL Coventry, UK;

email: wendland@maths.warwick.ac.uk 